\title{
Renormalization Group Method
}

\author{
A Thesis Submitted to the \\ College of Graduate Studies and Research \\ in Partial Fulfillment of the Requirements \\ for the degree of Master of Science \\ in the Department of Mathematics and Statistics \\ University of Saskatchewan \\ Saskatoon
}

By

Mehdi Rostamiforooshani

(C)Mehdi Rostamiforooshani, August 2013. All rights reserved. 


\section{Permission to Use}

In presenting this thesis in partial fulfilment of the requirements for a Postgraduate degree from the University of Saskatchewan, I agree that the Libraries of this University may make it freely available for inspection. I further agree that permission

for copying of this thesis in any manner, in whole or in part, for scholarly purposes may be granted by the professor or professors who supervised my thesis work or, in their absence, by the Head of the Department or the Dean of the College in which my thesis work was done. It is understood that any copying or publication or use of this thesis or parts thereof for financial gain shall not be allowed without my written permission. It is also understood that due recognition shall be given to me and to the University of Saskatchewan in any scholarly use which may be made of any material in my thesis.

Requests for permission to copy or to make other use of material in this thesis in whole or part should be addressed to:

Head of the Department of Mathematics and Statistics

142 McLean Hall

University of Saskatchewan

Saskatoon, Saskatchewan

Canada

S7N 5E6 


\section{ABStraCt}

Renormalization Group (RG) method is a general method whose aim is to globally approximate solutions to differential equations involving a small parameter. In this thesis, we will give an algorithm for the RG method to generate the RG equation needed in the process of finding an approximate solution for ODEs. In chapter 1, we have some introduction to perturbation theory and introducing some traditional methods in perturbation theory. In chapter 2 we compare the results of RG and other conventional methods using numerical or explicit methods. Thereafter, in chapter 3 , we rigorously compare the approximate solution obtained using the $\mathrm{RG}$ method and the true solution using two classes of system of ordinary differential equations. In chapter 4, we present a simplified RG method and apply it to the second order RG. In chapter 5 we briefly explain the first order Normal Form (NF) theory and then its relation to the RG method. Also a similar geometric interpretation for the RG equation and NF's outcome has been provided. In the Appendix, we have added definitions and proofs used in this thesis. The RG method is much more straightforward than other traditional methods and does not require prior information about the solutions. One begins with a naive perturbative expansion which already contains all the necessary information that we need to construct a solution. Using $\mathrm{RG}$, there is no need to asymptotically match the solutions in the overlapping regions, which is a key point in some other methods. In addition, the RG method is applicable to most of perturbed differential equations and will produce a closed form solution which is, most of the times, as accurate as or even more accurate than the solutions obtained by other conventional methods. 


\section{ACKNOWLEDGEMENTS}

There are four mathematicians who have greatly influenced my career path, whom I would like to sincerely thank. Foremost, I would like to thank my supervisors, Professors Jacek Szmigielski and Walid Abou Salem for great guidance and supports. As far as I know, my supervisors spent time to teach me and guide me more than any other supervisor in our department. Both of them are well-known in the department as excellent teachers who display enthusiasm, respect, and most of all patience to their colleagues and students. Thank you, Professor Abou Salem and thank you Professor Szmigielski, for the invaluable direction, motivation, advice, and financial support you provided during my time as a graduate student.

Secondly, I would like to thank Professors Majid Gazor and Mohammadreza Raoofi for their positive influences. When I was an undergraduate student, I took several courses with them. Their great research and teaching as well as my consultation from them, persuaded me to apply for this program. I also visited Professor Gazor in the second year of my program at the Isfahan University of Technology to learn Normal Form Theory from him. Thank you Professors Gazor and Raoofi for your helpful insights and advice.

Thank to the Head and Acting Head of the department, Professor Raj Srinivasan and Professor Chris Soteros, for providing additional financial support and ensuring me to complete my studies.

I thank my family especially my lovely wife, Najmeh, who is always one step ahead of me, being patient and providing me with constant support and serenity. 
I dedicate this work to my parents, Batool and Rajabali. 


\section{CONTENTs}

Permission to Use i

Abstract $\quad$ ii

Acknowledgements $\quad$ iii

Contents $\quad$ v

List of Tables $\quad$ vii

1 Introduction 1

2 The RG Method vs. Conventional Methods $\quad 7$

2.1 Rayleigh Oscillator . . . . . . . . . . . . . . . . . . 7

2.1.1 The Multiple Scale Analysis . . . . . . . . . . . . . . . . 7

2.1.2 The RG Method . . . . . . . . . . . . . . . . . . 10

2.2 The Mathieu Equation . . . . . . . . . . . . . . . . . . . 17

2.2.1 Multiple Scale Analysis Applied to the Mathieu Equation . . . 18

2.2.2 The RG Method Applied to the Mathieu Equation . . . . . . 23

2.3 A Simple Second Order ODE . . . . . . . . . . . . . . . . . . 28

2.3.1 The Boundary Layer Theory . . . . . . . . . . . . . . . . . 29

2.3.2 The RG Method Applied to a Simple Second Order ODE . . . 33

2.4 The Time-Independent Schrödinger Equation . . . . . . . . . . . 36

2.4.1 WKB Approximation Applied to the Time-Independent Schrödinger Equation . . . . . . . . . . . . . . 37

2.4.2 The RG Method Applied to the Time-Independent Schrödinger

Equation . . . . . . . . . . . . . . 43

2.5 Nonlinear Carrier Equation . . . . . . . . . . . . . . . 51

2.5.1 The Exact Solution . . . . . . . . . . . . . . . 53

2.5.2 The RG method Applied to the Carrier Equation . . . . . . . 54

3 A Rigorous Discussion of the RG Method 56

3.1 The Procedure of the RG Method . . . . . . . . . . . . . 56

3.2 Estimate the Error of RG Approximation . . . . . . . . . . . . 62

3.2.1 Estimate the RG approximation' Error (Oscillatory Case) . . 64

3.2.2 Estimate the RG approximation' Error (Dissipation Case) . . 70

3.3 Reproducing the RG Equation . . . . . . . . . . . . . . . . . 74 
4 Simplified RG Method and The Second Order RG 78

4.1 The Simplified Procedure . . . . . . . . . . . . . . . . . . . 78

4.2 Second Order RG Method . . . . . . . . . . . . . . . . . 82

5 The Relationship Between the Renormalization Group Method and the Normal Form Theory 96

5.1 The Normal Form Theory _. . . . . . . . . . . . . . . . . . 96

5.2 The Relation Between NF and RG . . . . . . . . . . . . . . 99

5.3 The Geometric Interpretations of $\mathrm{RG}$ and $\mathrm{NF} \ldots \ldots \ldots$

6 Appendix 108

6.1 Asymptotic Analysis (Local Analysis) . . . . . . . . . . . . . 108

6.2 Airy Functions . . . . . . . . . . . . . . . . . . . . 110

6.3 Perturbation Theory . . . . . . . . . . . . . . . . . . . 114

6.4 Boundary Layer Theory $\ldots \ldots \ldots$

6.5 WKB Theory . . . . . . . . . . . . . . . . . . . 117

6.6 Floquet Theory . . . . . . . . . . . . . . . . . . . . . . . . . . . . . 120

6.7 Mean Value Theorem . . . . . . . . . . . . . . . . . . . . . . . . . 124

6.8 Gronwall's Lemma . . . . . . . . . . . . . . . . . . . . . . . . 125

6.9 Differential Geometry . . . . . . . . . . . . . . . . . . 127 


\section{List OF TABLES}




\section{Chapter 1}

\section{INTRODUCTION}

Perturbation theory first appeared in one of the oldest branches of applied mathematics and physics, namely in celestial mechanics which is the study of the motions of the planets. For example, if there are only two gravitational bodies, the trajectories of both masses are ellipses with a common focal point located at the center of mass. But because there are other gravitational bodies, they modify, or "perturb", their motions. Perturbation theory originally refers to taking these modifications into account [1]. Nevertheless, the application of perturbation theory is not limited only to celestial mechanics. In general, perturbation theory is regarded as a collection of methods (iterative methods) which may be used to solve an equation- differential, polynomial or linear algebraic equation- which contains a small parameter, say $\epsilon$ $[1,3]$. Sometimes, to approximate solutions to an equation, it is helpful to introduce $\epsilon$ somewhere in the equation and to use a perturbation method. After approximation is carried out, setting $\epsilon=1$ recovers the original problem. Perturbation methods are not always applicable! The practical condition is whether the problem is becoming easier for $\epsilon=0$.

Let the perturbed differential equation ${ }^{1}$

$$
\frac{d^{2} y}{d x^{2}}=F\left(x, y, \frac{d y}{d x}, \epsilon\right)
$$

\footnotetext{
${ }^{1}$ A differential equation involving a small parameter.
} 
be given, for some function $F$. If the naive perturbative expansion,

$$
\sum_{n=0}^{\infty} \epsilon^{n} y_{n}
$$

converges to the actual solution of (1.1), the problem is regular. If either the naive perturbative expansion does not converge to the solutions or if it does, it has a vanishing radius of convergence, the problem is singular [3]. In this thesis, we will briefly present three traditional singular perturbation methods as well as introduce relatively new, the RG method approach as developed by Goldenfeld et. al., all of which are used to approximate the solutions of singular ordinary differential equations involving a small parameter. One of the methods is the Boundary Layer Theory, which can be traced to an investigation of the motion of a fluid with a very small viscosity, studied by Prandtl [4]. The boundary layer theory is a traditional method which can be applied to those equations whose highest derivative term is multiplied by a small parameter. Such equations have boundary layer structure, having a narrow region in which the solution has an abrupt change. In the Boundary Layer Theory, we separate the solution into different solutions valid in different regions of interest and then match them together in the intermediate region. The process of matching the solutions is challenging and presents a series of difficulties in the implementation of the method. The next perturbation method is Wentzel- Kramers-Brillouin-Jeffreys method, known as the WKB, WKBJ, exponential, or adiabatic approximation. This method was invented and developed in the context of the Schrödinger equation and quantum theory $[7,8,9,10]$. In WKB theory, we postulate the solution as an exponential function and then by substituting it into the original equation we try to find the exponent. The other conventional perturbation method is the Multiple Scale Analysis which is attributed to Poincare [5] and was developed in a more general version in 1949, by Lighthill [6]. This method is applicable to those equations which contain secular terms in their naive perturbative expansions. In the Multiple Scale Analysis, we introduce another independent variable(s) (or time scale(s)) to absorb 
the secular terms. However there are no general rules as to how to define a new time scale(s) which sometimes leads to tedious computations. These methods have disadvantages and limitations to use. However, for many years, they were the only methods available and, in fact, these methods are still in use. Scientists have been striving to find a unified and more straightforward method to approximate solutions of differential equations. In 1994, Goldenfeld and his coworkers realized that the renormalization group method, which is a certain method and was previously used in quantum and statistical mechanics, can be carried over to solve perturbed differential equations[11, 12]. This method does not have those limitations and has been improved and simplified during last two decades. Using the renormalization group (RG) method, we neither need to asymptotically match inner and outer solutions in case of the boundary layer or WKB theories nor define a new time scale in problems which contain secular terms. Also the procedure is the same for every example and gives a closed form formula for the solutions. In the RG method, we first begin with a naive perturbative expansion with respect to $\epsilon$. Then by inserting it into the equation, we obtain a sequence of simpler differential equations. For first order correction, we only need to study two first terms. Usually, the convergence of the series breaks down for large values of the independent variable $t$, because of existence of a secular term (a term which usually contains the factor $\left(t-t_{0}\right)^{m}$, for some positive $m$ ). Other bounded terms are called nonresonant terms. The method suggests to absorb the secular term to find a new differential equation(s), called the RG equation.

After inventing the RG method, many people have worked in this area trying to simplify or to interpret this method. One significant progress was achieved by Oono and Nozaki who found which terms in the expansion have the main contribution to result in the RG equation (See [18]). They conjectured that "resonant secular terms" are the only terms which contain all information one needs to determine the $\mathrm{RG}$ equation. A resonant secular term is a term which involves the multiplication 
of the leading order term (i.e. solution to the equation when $\epsilon=0$ ) and the factor $\left(t-t_{0}\right)^{m}$, for some positive $m$. On the other hand, nonresonant secular terms are those secular terms which are not resonant secular. This means that, to find the RG equation, we only should care about the resonant secular terms, while nonresonant secular terms are automatically taken care of. This fact was shown for a large class of differential equations in 2012 by Oono and Shiwa [19]. In this thesis we will not study this simplification except for a brief remark in chapter 3. In the three examples in the fourth chapter, we demonstrate their result. It must be noted that, in a rigorous approximation, all terms of the expansion must be considered even nonresonant terms (see chapter 3); it is not enough to have the resonant secular terms in the expansion because even bounded terms will play role in approximating for larger time. ${ }^{2}$

Now we turn to a more detailed description of the content of this thesis. In the second chapter, we solve 5 examples using the traditional singular perturbation methods and then we apply the RG method to solve the same examples:

\begin{tabular}{|l|l|l|}
\hline Number & The Perturbed ODE & The Traditional Method \\
\hline 1 & The Rayleigh Oscillator & The Multiple Scale Analysis \\
\hline 2 & The Mathieu Equation & The Multiple Scale Analysis \\
\hline 3 & A Simple Linear BVP & The Boundary Layer Theory \\
\hline 4 & The Schrödinger Equation & The WKB Approximation \\
\hline 5 & The Nonlinear Carrier Equation & The Exact Solution \\
\hline
\end{tabular}

After applying the traditional methods to the examples we apply the RG method to each and thereafter compare the results of the conventional methods with the results of the RG method using numerical simulations.

\footnotetext{
${ }^{2}$ The best reference book (practical book) of singular perturbation is " C. M. Bender and S. A. Orszag, Advanced Mathematical Methods for Scientists and Engineers". The recently published book, "The Nonlinear World" by Yoshitsugu Oono (2012), is the best reference to find intuitive explanation of the RG method [20].
} 
The numerical figures in this chapter (and chapter four) have been plotted using the MATLAB software. In most of them, the main command is "ode45" (except for the figures 2.7 and 2.8). This command implements 4th or 5th order Runge-Kutta (RK) method. The advantages of RK are: 1) High order of accuracy, 2) Longer stability region than explicit methods such as Forward Euler method (FE)

In the third chapter, in section one, we consider a general autonomous system of differential equations, which covers most of examples in chapter 1 and the examples in the papers [11] and [12]. Then we apply the RG method to this system and derive the $R G$ equation. In the next section, we estimate the actual solution with the RG's outcome and show that the RG's outcome converges to the exact solution. Most of the material contained in this chapter follow the paper of Ziane [15]. Finally, we make the observation that, for an autonomous system of differential equations, the RG method and the Multiple Scale Analysis produce identical results. In this chapter, three other ways of reproducing the RG equation are given.

In the fourth chapter, we study two issues: In the first section, we present a simplified RG method suggested by R. DeVille et. al [16]. In the second section, we present three examples and show how one can apply RG up to the second order; we use the simplified RG method to find the second order RG approximation. There are several figures which demonstrate that the second order RG improves the accuracy of the first order.

In the fifth chapter, we use the simplified RG method, which is equivalent to the classical RG method, to observe the relationship between the RG method and the Normal Form (NF) theory. In this subsection also we discuss the pros and cons of the RG and NF theories. At the end of this chapter, we present a geometric interpretation of the RG method [16] and the NF theory.

Finally, in the Appendix, there are some theorems and definitions that makes the reading of this dissertation easier. Also a brief discussion of the local asymptotic 
analysis as well as global analysis is presented. The perturbative methods, WKB approximation and boundary layer which are used in the first chapter, are introduced in the Appendix as well. 


\section{Chapter 2 \\ The RG Method vs. Conventional Meth- ODS}

\subsection{Rayleigh Oscillator}

The following equation is called the Rayleigh Oscillator equation:

$$
\ddot{y}(t)+y(t)=\epsilon\left(\dot{y}(t)-\frac{1}{3} \dot{y}(t)^{3}\right), \quad y(0)=0, \dot{y}(0)=2 a, t>0,
$$

where $\dot{y}$ means the derivative with respect to time and $a$ is an arbitrary real parameter.

This equation consists of the harmonic oscillator part $\ddot{y}+y$ plus a velocity dependent perturbation $\epsilon\left(\dot{y}-\frac{1}{3} \dot{y}^{3}\right)$.

In the forthcoming, we will explain two singular perturbation methods, the multiple scale analysis and the RG method by applying them to the Rayleigh equation.

\subsubsection{The Multiple Scale Analysis}

We are going to apply the multiple scale analysis to the equation (2.1), see [3] chapter 11.

To begin with, we seek a perturbative expansion of the solution of (2.1) in the form

$$
\begin{array}{r}
y(t)=y_{0}(t)+\epsilon y_{1}(t)+\mathcal{O}\left(\epsilon^{2}\right), \quad y_{0}(0)=0, \quad \dot{y}_{0}(0)=2 a, \\
y_{i}(0)=0, \quad \dot{y}_{i}(0)=0, i \geq 1,
\end{array}
$$


where we imposed the initial condition on the leading term.

We differentiate this expansion term by term and substitute the result into (2.1) to obtain

$$
y_{0}+\ddot{y_{0}}+\epsilon\left(y_{1}+\ddot{y_{1}}\right)+\mathcal{O}\left(\epsilon^{2}\right)=\epsilon\left(\dot{y}_{0}-\frac{1}{3} \dot{y}_{0}{ }^{3}+\mathcal{O}(\epsilon)\right)=\epsilon\left(\dot{y}_{0}-\frac{1}{3} \dot{y}_{0}{ }^{3}\right)+\mathcal{O}\left(\epsilon^{2}\right) .
$$

Now comparing the powers of $\epsilon$ gives a sequence of equations

$$
\begin{array}{ll}
\ddot{y_{0}}+y_{0}=0, & y_{0}(0)=0, \dot{y}_{0}(0)=2 a, \\
\ddot{y_{1}}+y_{1}=\dot{y_{0}}-\frac{1}{3} \dot{y}^{3}, & y_{1}(0)=\dot{y}_{1}(0)=0, \\
\ddot{y_{2}}+y_{2}=\dot{y}_{1}\left(1-\dot{y}_{0}{ }^{2}\right), & y_{2}(0)=\dot{y}_{2}(0)=0,
\end{array}
$$

and so on.

To approximate up to the order of $\epsilon$, we only need to solve (2.2a) as well as $(2.2 \mathrm{~b})$. The specific solutions to them are

$$
y_{0}(t)=2 a \sin t
$$

and,

$$
\begin{aligned}
& y_{1}(t)=a \sin ^{3} t-2 a^{2} \sin ^{5} t+\left(3 a^{3}-a\right) t \cos t+ \\
& \qquad \frac{1}{2}\left(a-4 a^{3}\right) \sin 2 t \cos t+\frac{a^{3}}{4} \sin 4 t \cos t .
\end{aligned}
$$

The term $t \cos t$ in (2.4) is called a "secular term". When compared to the leading term, it becomes significant for large time. Observe that this secular term becomes large when time is of the order of $1 / \epsilon$.

We use the Multiple Scale Analysis to deal with such secular terms. We define a new independent time scale $\tau=\epsilon t$, and instead of the previous expansion, we consider a new one

$$
y(t)=Y_{0}(t, \tau)+\epsilon Y_{1}(t, \tau)+\mathcal{O}\left(\epsilon^{2}\right) .
$$

Note that we here suppose that $Y_{0}$ and $Y_{1}$ are functions of $t$ and $\tau$. 
Since $\frac{d \tau}{d t}=\epsilon$, we find

$$
\begin{aligned}
& \dot{y}=\frac{\partial Y_{0}}{\partial t}+\epsilon\left(\frac{\partial Y_{0}}{\partial \tau}+\frac{\partial Y_{1}}{\partial t}\right)+\mathcal{O}\left(\epsilon^{2}\right), \\
& \ddot{y}=\frac{\partial^{2} Y_{0}}{\partial t^{2}}+\epsilon\left(2 \frac{\partial^{2} Y_{0}}{\partial t \partial \tau}+\frac{\partial^{2} Y_{1}}{\partial t^{2}}\right)+\mathcal{O}\left(\epsilon^{2}\right) .
\end{aligned}
$$

Substituting (2.5b) into (2.1) and comparing both sides result in

$$
\begin{aligned}
\frac{\partial^{2} Y_{0}}{\partial t^{2}}+Y_{0} & =0 \\
Y_{1}+\left(2 \frac{\partial^{2} Y_{0}}{\partial t \partial \tau}+\frac{\partial^{2} Y_{1}}{\partial t^{2}}\right) & =\frac{\partial Y_{0}}{\partial t}-\frac{1}{3}\left(\frac{\partial Y_{0}}{\partial t}\right)^{3} .
\end{aligned}
$$

The solution to $(2.6 \mathrm{a})$ is

$$
Y_{0}(t, \tau)=A(\tau) e^{i t}+A^{*}(\tau) e^{-i t}
$$

Now the right hand side of $(2.6 \mathrm{~b})$ becomes

$$
\left(-2 A^{\prime}+A-A^{2} A^{*}\right) i e^{i t}+\left(-2 A^{* \prime}+A^{*}-A A^{* 2}\right)(-i) e^{-i t}+\frac{1}{3} i A^{3} e^{3 i t}+\frac{1}{3} A^{* 3} e^{-3 i t},
$$

where $A^{\prime}=\frac{d A}{d \tau}$.

The secular terms usually occur when the inhomogeneous term contains a solution of the homogeneous equation (2.2b). To avoid the appearance of secular terms, we equate the coefficients of $e^{i t}$ and $e^{-i t}$ to zero (since they are solutions to the homogeneous equation). This yields a condition on $A(\tau)$ which allows us to find it:

$$
\begin{aligned}
-2 \frac{d A}{d \tau}+A-A^{2} A^{*} & =0 \\
-2 \frac{d A^{*}}{d \tau}+A^{*}-A A^{* 2} & =0
\end{aligned}
$$

Since $(2.8 \mathrm{~b})$ is complex conjugate of $(2.8 \mathrm{a})$, it is enough to solve

$$
-2 \frac{d A}{d \tau}+A-A^{2} A^{*}=0 .
$$


Here it is convenient to use polar coordinates to solve $(2.9): A=R(\tau) e^{i \theta(\tau)}$ for some real functions $R(\tau)$ and $\theta(\tau)$. Then

$$
-2 \frac{d R}{d \tau} e^{i \theta}-2 i R \frac{d \theta}{d \tau} e^{i \theta}+R e^{i \theta}-R^{3}\left(e^{2 i \theta} e^{-i \theta}\right)=e^{i \theta}\left(-2 \frac{d R}{d \tau}-2 R \frac{d \theta}{d \tau} i+R-R^{3}\right)=0
$$

Thus

$$
\begin{aligned}
\frac{d \theta}{d \tau} & =0 \\
\frac{d R}{d \tau} & =\frac{1}{2}\left(R-R^{3}\right)
\end{aligned}
$$

Solving (2.10)s gives

$$
\theta(\tau)=c_{0}, \text { and } R(\tau)=\frac{1}{\sqrt{1+c_{1} e^{-\tau}}}
$$

To find $c_{0}$ and $c_{1}$ we use initial conditions for $Y_{0}$. The final answer is

$$
R(0)=a, \theta(0)=-\frac{1}{2} \pi
$$

Thus we have the following nice formula for the leading behavior (or the zeroth order) of the Rayleigh Oscillator:

$$
y_{0}(t)=\frac{2 a \sin t}{\sqrt{e^{-\epsilon t}+a^{2}\left(1-e^{-\epsilon t}\right)}} .
$$

\subsubsection{The RG Method}

Now we introduce the RG Method (or CGO-RG) by applying it to the Rayleigh equation, see [12]. Before starting, we will give an algorithm for the RG method to a general example.

- STEP I. The naive expansion. First we assume a perturbative expansion for the solution to the ODE: 


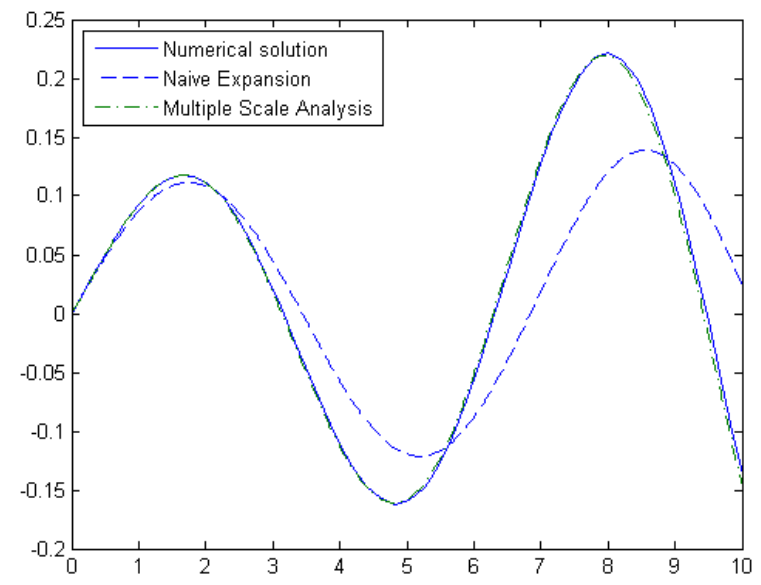

Figure 2.1: Figure of solution to Rayleigh Oscillator Equation with 3 methods where we have taken $\epsilon=.2$ and $a=.05$.

$$
y(t)=\sum_{n=0}^{\infty} \epsilon^{n} y_{n}(t) .
$$

We truncate the expansion, say $k$ th order, and insert it into the equation. We equate the coefficients of $1, \epsilon, \ldots, \epsilon^{k}$ order by order. Hence we are left with $k+1$ differential equations. We then solve and substitute them into the expansion.

- STEP II. We absorb the integral constants of higher orders into the integral constant of $y_{0}$.

- STEP III. We investigate the secular term(s) and try to absorb the secularity(ies). Secular terms are those terms which contain or result in the factor $\left(t-t_{0}\right)$. We introduce a new parameter, $\sigma$, such that $t-t_{0}=(t-\sigma)+\left(\sigma-t_{0}\right)$.

- STEP IV. We absorb terms which contain $\sigma-t_{0}$ into the integral constant of $y_{0}$. (We may do this step and the second one at the same time.)

- STEP V. Since we do not have $\sigma$ in the original equation, the final solution must be independent of $\sigma$. In fact, $\frac{\partial y}{\partial \sigma}=0$. This condition results in new equation(s) 


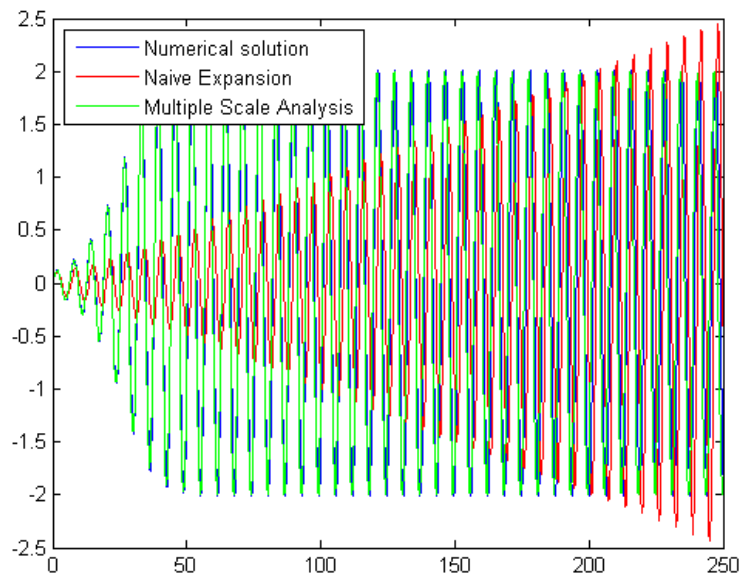

Figure 2.2: This figure shows how well multiple scale analysis approximates the solutions for large times. Note that the amplitude of naive expansion grows linearly in time.

called the RG equation(s).

Recall the Rayleigh Oscillator Equation

$$
\frac{\partial^{2} y}{d t^{2}}+y=\epsilon\left(\frac{d y}{d t}-\frac{1}{2}\left(\frac{d y}{d t}\right)^{3}\right)
$$

To approximate up to and including the first order, we make the Ansatz

$$
\ddot{y}(t)+y(t)=\epsilon\left(\dot{y}(t)-\frac{1}{3} \dot{y}(t)^{3}\right), \quad y(0)=0, \dot{y}(0)=2 a, t>0,
$$

Now, we substitute this into (2.12) and equate the coefficients order by order. Then we get

$$
\begin{aligned}
& \frac{d^{2} y_{0}}{d t^{2}}+y_{0}=0 \\
& \frac{d^{2} y_{1}}{d t^{2}}+y_{1}=\frac{d y_{0}}{d t}-\frac{1}{3}\left(\frac{d y_{0}}{d t}\right)^{3}
\end{aligned}
$$

The general solution to $(2.13 \mathrm{a})$ is

$$
y_{0}(t)=A_{0} \sin t+B_{0} \cos t
$$


or

$$
y_{0}(t)=R_{0} \sin \left(t+\theta_{0}\right)
$$

where $A_{0}=R_{0} \cos \theta_{0}$ and $B_{0}=-R_{0} \sin \theta_{0}$. Note that either $A_{0}$ and $B_{0}$ or $R_{0} \cos \theta_{0}$ and $R_{0} \sin \theta_{0}$ are determined by initial conditions.

We need a particular solution to $(2.13 \mathrm{~b})$ to find the general solution. Since $W(\sin (t), \cos (t))=-1,(W$ represents Wronskian of two solutions of homogeneous part of (2.13b)), we have:

$$
y_{p}=u_{1} y_{1}^{1}+u_{2} y_{1}^{2},
$$

such that, $y_{1}^{1}=\sin t$ and $y_{1}^{2}=\cos t$ and

$$
\begin{aligned}
& u_{1}^{\prime}=\cos t\left(\left(R_{0}-\frac{R_{0}^{3}}{4}\right) \cos \left(t+\theta_{0}\right)-\frac{R_{0}^{3}}{12} \cos 3\left(t+\theta_{0}\right)\right), \\
& u_{2}^{\prime}=-\sin t\left(\left(R_{0}-\frac{R_{0}^{3}}{4}\right) \cos \left(t+\theta_{0}\right)-\frac{R_{0}^{3}}{12} \cos 3\left(t+\theta_{0}\right)\right) .
\end{aligned}
$$

Using trigonometric relations and integrating yield

$$
y_{p}=\frac{R_{0}}{2}\left(1-\frac{R_{0}^{2}}{4}\right)\left(t-t_{0}\right) \sin \left(t+\theta_{0}\right)+\frac{R_{0}^{3}}{96} \cos 3\left(t+\theta_{0}\right) .
$$

Thus

$$
\begin{aligned}
y(t)=R_{0} \sin \left(t+\theta_{0}\right)+ & \epsilon\left(A \sin \left(t+\theta_{0}\right)+B \cos \left(t+\theta_{0}\right)+\right. \\
& \left.\frac{R_{0}}{2}\left(1-\frac{R_{0}^{2}}{4}\right)\left(t-t_{0}\right) \sin \left(t+\theta_{0}\right)+\frac{R_{0}^{3}}{96} \cos 3\left(t+\theta_{0}\right)\right)+\mathcal{O}\left(\epsilon^{2}\right),
\end{aligned}
$$

where $A$ and $B$ are integral constants of $y_{1}$.

Now we add and subtract a free time scale, $\sigma$, in the factor $t-t_{0}$ and rewrite the equation

$$
\begin{aligned}
& y(t)=R_{0} \sin \left(t+\theta_{0}\right)+\epsilon\left(\left(A+\frac{R_{0}}{2}\left(1-\frac{R_{0}^{2}}{4}\right)\left(\sigma-t_{0}\right)\right) \sin \left(t+\theta_{0}\right)+\right. \\
& \left.B \cos \left(t+\theta_{0}\right)+\frac{R_{0}}{2}\left(1-\frac{R_{0}^{2}}{4}\right)(t-\sigma) \sin \left(t+\theta_{0}\right)+\frac{R_{0}^{3}}{96} \cos 3\left(t+\theta_{0}\right)\right)+\mathcal{O}\left(\epsilon^{2}\right) .
\end{aligned}
$$


Now the aim is to renormalize the term containing $\sigma-t_{0}$ as well as a general solution to the homogeneous part of (2.13b). First, let us define $a\left(\sigma, t_{0}\right), b\left(\sigma, t_{0}\right)$, $R(\sigma)$ and $\theta(\sigma)$

$$
\begin{aligned}
R_{0}\left(t_{0}\right) & =R(\sigma)+\epsilon a\left(\sigma, t_{0}\right) R(\sigma)+\mathcal{O}\left(\epsilon^{2}\right), \\
\theta\left(t_{0}\right) & =\theta(\sigma)+\epsilon b\left(\sigma, t_{0}\right)+\mathcal{O}\left(\epsilon^{2}\right) .
\end{aligned}
$$

Notice that $R_{0}$ and $\theta_{0}$ are not functions! If the initial time is arbitrary, the integral constants depend on the initial time. Since we have not imposed the initial conditions yet, we may assume that the initial time is arbitrary.

Then we substitute (2.15) into (2.14) keeping terms up to the order of $\epsilon\left(\mathcal{O}\left(\epsilon^{2}\right)\right.$ at the end of (2.14) absorbs the rest)

$$
\begin{gathered}
y(t)=(R+\epsilon a R) \sin (t+\theta+b \epsilon)+ \\
\epsilon\left(A+\frac{R}{2}\left(1-\frac{R^{2}}{4}\right)\left(\sigma-t_{0}\right)\right) \sin (t+\theta+b \epsilon)+\epsilon B \cos (t+\theta+b \epsilon)+ \\
\quad \frac{\epsilon R}{2}\left(1-\frac{R^{2}}{4}\right)(t-\sigma) \sin (t+\theta+b \epsilon)+\frac{\epsilon R^{3}}{96} \cos 3(t+\theta+b \epsilon)+\mathcal{O}\left(\epsilon^{2}\right) .
\end{gathered}
$$

where for simplicity $R=R(\sigma), \theta=\theta(\sigma), a=a\left(\sigma, t_{0}\right)$ and $b=b\left(\sigma, t_{0}\right)$.

Using Taylor series, $\sin (t+\theta+b \epsilon) \sim \sin (t+\theta)+b \epsilon \cos (t+\theta)$ and $\cos (t+\theta+b \epsilon) \sim$ $\cos (t+\theta)-b \epsilon \sin (t+\theta)$ we obtain

$$
\begin{gathered}
y(t)=R \sin (t+\theta)+b \epsilon R \cos (t+\theta)+\epsilon a R \sin (t+\theta+b \epsilon)+ \\
\epsilon\left(A+\frac{R}{2}\left(1-\frac{R^{2}}{4}\right)\right)\left(\sigma-t_{0}\right) \sin (t+\theta+b \epsilon)+\epsilon B \cos (t+\theta)+ \\
\frac{\epsilon R}{2}\left(1-\frac{R^{2}}{4}\right)(t-\sigma) \sin (t+\theta)+\frac{\epsilon R^{3}}{96} \cos 3(t+\theta)+\mathcal{O}\left(\epsilon^{2}\right) .
\end{gathered}
$$

Now set

$$
\begin{aligned}
& b=\frac{-B}{R}, \\
& a=\frac{-A}{R}-\frac{1}{2}\left(1-\frac{R^{2}}{2}\right)\left(\sigma-t_{0}\right) .
\end{aligned}
$$


Hence

$$
y(t)=R \sin (t+\theta)+\epsilon R\left(\frac{1}{2}\left(1-\frac{R^{2}}{4}\right)(t-\sigma) \sin (t+\theta)+\frac{R^{2}}{96} \cos 3(t+\theta)\right)+\mathcal{O}\left(\epsilon^{2}\right) .
$$

The original equation (2.12) did not contain $\sigma$ while (2.16) does. This implies that $\frac{\partial y}{\partial \sigma}=\mathcal{O}\left(\epsilon^{2}\right)$. Thus

$$
\begin{gathered}
\frac{\partial y}{\partial \sigma}=\frac{d R}{d \sigma} \sin (t+\theta)+\frac{d \theta}{d \sigma} R \cos (t+\theta)+\epsilon \frac{d R}{d \sigma}\left(\frac{1}{2}\left(1-\frac{R^{2}}{4}\right)(t-\sigma) \sin (t+\theta)-\right. \\
\left.\frac{R^{3}}{96} \cos 3(t+\theta)\right)+\epsilon R\left(\frac{-1}{4} \frac{d R}{d \sigma}(t-\sigma) \sin (t+\theta)-\frac{1}{2}\left(1-\frac{R^{2}}{4}\right) \sin (t+\theta)+\right. \\
\left.\frac{1}{2} \frac{d \theta}{d \sigma}\left(1-\frac{R^{2}}{4}\right) \cos (t+\theta)+\frac{R}{48} \frac{d R}{d \sigma} \cos 3(t+\theta)-\frac{R^{2}}{32} \frac{d \theta}{d \sigma} \sin 3(t+\theta)\right)=\mathcal{O}\left(\epsilon^{2}\right) .
\end{gathered}
$$

We observe that

$$
\frac{d R}{d \sigma} \sin (t+\theta)+\frac{d \theta}{d \sigma} R \cos (t+\theta)=\mathcal{O}(\epsilon)
$$

Since $\sin (t+\theta)$ and $\cos (t+\theta)$ are linearly independent we get

$$
\begin{aligned}
\frac{d R}{d \sigma} & =\mathcal{O}(\epsilon), \\
\frac{d \theta}{d \sigma} & =\mathcal{O}(\epsilon) .
\end{aligned}
$$

Inserting (2.18) into (2.17) gives

$$
\frac{d R}{d \sigma} \sin (t+\theta)+\frac{d \theta}{d \sigma} R \cos (t+\theta)-\frac{\epsilon R}{2}\left(1-\frac{R^{2}}{4}\right) \sin (t+\theta)=\mathcal{O}\left(\epsilon^{2}\right) .
$$

Note that $\mathcal{O}\left(\epsilon^{2}\right)$ has absorbed terms of order $\epsilon^{2}$.

Therefore

$$
\begin{aligned}
\frac{d R}{d \sigma} & =\frac{\epsilon R}{2}\left(1-\frac{R^{2}}{4}\right)+\mathcal{O}\left(\epsilon^{2}\right) \\
\frac{d \theta}{d \sigma} & =\mathcal{O}\left(\epsilon^{2}\right)
\end{aligned}
$$


(2.19) are first order differential equations that can be solved using elementary methods up to some error:

$$
\begin{aligned}
\tilde{R}(\sigma) & =\frac{\tilde{R}(0)}{\sqrt{e^{-\epsilon \sigma}+\frac{1}{4} \tilde{R}(0)^{2}\left(1-e^{-\epsilon \sigma}\right)}}, \\
\tilde{\theta}(\sigma) & =\tilde{\theta}(0) .
\end{aligned}
$$

To find the error of above approximation to the true solution of the RG equations (2.19), we can use the Gronwall lemma 6.27. Before applying the Gronwall lemma, notice that if $\sigma \in[a, b]$, for some real numbers $a$ and $b$, there are constants $c_{1}$ and $c_{2}$ such that

$$
\begin{aligned}
\frac{d R}{d \sigma}-\frac{\epsilon R}{2}\left(1-\frac{R^{2}}{4}\right) & \leq c_{1} \epsilon^{2}, \\
\frac{d \theta}{d \sigma} & \leq c_{2} \epsilon^{2} .
\end{aligned}
$$

Now let us define $w_{1}=R-\tilde{R}$. Hence, for some positive $c_{0}$, independent of $\epsilon$

$$
\frac{d w_{1}}{d \sigma} \leq c_{0} \epsilon w_{1}+c_{1} \epsilon^{2} .
$$

Now applying Gronwall's lemma yields

$$
w_{1} \leq e^{c_{0} \epsilon \sigma} \int_{0}^{\sigma} c_{1} \epsilon^{2} e^{-c_{0} \epsilon \tau} d \tau=\frac{c_{1}}{c_{0}} \epsilon\left(e^{c_{0} \epsilon \sigma}-1\right)
$$

Hence

$$
w_{1}=\mathcal{O}\left(\sigma \epsilon^{2}\right)
$$

Doing the same procedure for $w_{2}=\theta-\tilde{\theta}$, we have $w_{2}=\mathcal{O}\left(\sigma \epsilon^{2}\right)$. Therefore

$$
\begin{aligned}
& R(\sigma)=\frac{R(0)}{\sqrt{e^{-\epsilon \sigma}+\frac{1}{4} R(0)^{2}\left(1-e^{-\epsilon \sigma}\right)}}+\mathcal{O}\left(\sigma \epsilon^{2}\right), \\
& \theta(\sigma)=\theta(0)+\mathcal{O}\left(\sigma \epsilon^{2}\right) .
\end{aligned}
$$

Since the equation (2.12) does not depend on $\sigma$, we have a freedom to choose a relevant $\sigma$. Equation (2.16) suggests setting $\sigma=t$ in order to renormalize the secular term. Then we have:

$$
y(t)=R(t) \sin (t+\theta(t))+\frac{\epsilon R^{3}(t)}{96} \cos 3(t+\theta(t))+\mathcal{O}\left(t \epsilon^{2}\right) .
$$


where:

$$
\begin{aligned}
& R(t)=\frac{R(0)}{\sqrt{e^{-\epsilon t}+\frac{1}{4} R(0)^{2}\left(1-e^{-\epsilon t}\right)}}+\mathcal{O}\left(t \epsilon^{2}\right), \\
& \theta(t)=\theta(0)+\mathcal{O}\left(t \epsilon^{2}\right)
\end{aligned}
$$

Now we impose the initial conditions we had in the beginning of the section. Hence the zeroth order becomes

$$
y_{0}(t)=\frac{2 a \sin t}{\sqrt{e^{-\epsilon t}+a^{2}\left(1-e^{-\epsilon t}\right)}} .
$$

Comparing (2.23) to (2.24) implies that, in the case of the Rayleigh Oscillator, the result of the RG method and multiple scale analysis are identical (here up to the zeroth order). In his paper [21], Chiba proved that the multiple scale analysis and the RG method are equivalent or produce the identical results for the ordinary differential equations. A simple proof for the case of autonomous system of differential equations will be presented in the remark (3.6).

\subsection{The Mathieu Equation}

In this section we are going to apply the multiple scale analysis as well as the RG method to approximate the solutions to the Mathieu equation. The Mathieu equation is a linear second order initial value problem with a $2 \pi$ periodic coefficient:

$$
\ddot{y}+(a+2 \epsilon \cos t) y=0
$$

where $a$ is a positive parameter.

Notice that the Mathieu equation can be written as

$$
\left[\begin{array}{c}
\dot{x}_{1} \\
\dot{x}_{2}
\end{array}\right]=\left[\begin{array}{ll}
0 & 1 \\
a & 0
\end{array}\right]\left[\begin{array}{l}
x_{1} \\
x_{2}
\end{array}\right]+2 \epsilon \cos (t)\left[\begin{array}{l}
0 \\
1
\end{array}\right],
$$


where $x_{1}=y$ and $x_{2}=\dot{y}$.

The following theorem states a general form for the solutions to a differential equation whose coefficients are $T$-periodic, see the Appendix 6.6 for the proof.

Theorem 2.1. Floquet Theorem

Consider the linear system of equations

$$
\dot{x}(t)=A(t) x(t)
$$

where $A(t)$ is a T-periodic $n \times n$ matrix of coefficients and $x(t) \in \mathbb{C}^{n}$. Then there exists a solution to the system of the form $x(t)=e^{\mu t} \phi(t)$, for some complex number $\mu$ and some T-periodic vector function $\phi$.

According to this theorem, we expect the general solution to the Mathieu equation of the form $e^{\mu_{1} t} \phi_{1}(t)+e^{\mu_{2} t} \phi_{2}(t)$.

\subsubsection{Multiple Scale Analysis Applied to the Mathieu Equa- tion}

We are going to apply the multiple scale analysis to the Mathieu equation, see [3], chapter 11 .

To apply the multiple scale analysis, we need to find out whether any secular term(s) exists or not, and if it does, how we should define the new time scale(s). Similar to what we did in the case of the Rayleigh equation, we seek a naive perturbative expansion of the solution to (2.25):

$$
y(t)=y_{0}(t)+\epsilon y_{1}(t)+\mathcal{O}\left(\epsilon^{2}\right)
$$

By taking the derivative of (2.27) two times and then by substituting it into (2.25) we get

$$
\ddot{y}_{0}+a y+\epsilon\left(\ddot{y}_{1}+a y_{1}+2 y_{0} \cos t\right)=\mathcal{O}\left(\epsilon^{2}\right) .
$$


Hence

$$
\begin{aligned}
\ddot{y}_{0}(t)+a y_{0}(t) & =0, \\
\ddot{y}_{1}(t)+a y_{1}(t) & =-2 y_{0}(t) \cos t, \\
\vdots & \\
\ddot{y}_{n}(t)+a y_{n}(t) & =-2 y_{n-1}(t) \cos t, n>2 .
\end{aligned}
$$

To approximate up to and including $\mathcal{O}(\epsilon)$, we are only interested in the general solutions of (2.29a) and (2.29b). The general solution to (2.29a) is

$$
y_{0}=A_{0} e^{\sqrt{a} i t}+A_{0}^{*} e^{-\sqrt{a} i t} .
$$

Now, to find the general solution to $(2.29 \mathrm{~b})$, we first need to substitute (2.25) into $(2.29 \mathrm{~b})$ :

$$
\ddot{y}_{1}+a y_{1}(t)=-A_{0} e^{(\sqrt{a}+1) i t}-A_{0} e^{(\sqrt{a}-1) i t}+c . c,
$$

where $c . c$ is complex conjugate of $-A_{0} e^{(\sqrt{a}+1) i t}-A_{0} e^{(\sqrt{a}-1) i t}$.

Note that for some values of $a$ the right hand side of (2.31) may contain terms which are solutions to the homogeneous part. Such terms will lead to secular term(s). Consequently, there will exist secular term(s) if

$$
\sqrt{a} \pm 1= \pm \sqrt{a}
$$

Thus if $a=\frac{1}{4}$ a secular term will appear. However, the higher order corrections will result in more restrictions on $a$ if secular terms are to be avoided. Let's consider $n$th recursive nonhomogeneous differential equation for the naive expansion

$$
\ddot{y}_{n}(t)+a y_{n}(t)=-2 \cos (t) y_{n-1}(t)
$$

The right hand side of $(2.33)$ contains terms $e^{ \pm(\sqrt{a} \pm n) i t}$. This can be proven using induction on $n$. To use induction one needs the fact that if the right hand side of (2.33) contains $e^{ \pm(\sqrt{a} \pm m) i t}$, for some $m$ such that $a \neq \frac{m^{2}}{4}$, then a particular solution 
is $c e^{ \pm(\sqrt{a} \pm m) i t}$. Thus to have a valid approximation up to $\mathcal{O}\left(\epsilon^{n}\right)$, we must have the conditions $a \neq \frac{n^{2}}{4}, n \in \mathbb{N}$.

CASE I) $a \neq \frac{n^{2}}{4}$, for $n>0$.

In this case, for sufficiently small values of $\epsilon$, the naive perturbative expansion approximates well the solution (see the Figure (2.3)). Thus

$$
\begin{array}{r}
y(t)=\left(A_{0} e^{\sqrt{a} i t}+c . c\right)+\epsilon\left(e ^ { i \sqrt { a } t } \left(A_{1}+A_{0}\left(\frac{1}{1+2 \sqrt{a}} e^{i t}+\right.\right.\right. \\
\left.\left.\left.\frac{1}{1-2 \sqrt{a}} e^{-i t}\right)\right)+c . c\right)+\mathcal{O}\left(\epsilon^{2}\right),
\end{array}
$$

where $A_{1}=-A_{0}\left(\frac{1}{1+2 \sqrt{a}}+\frac{1}{1-2 \sqrt{a}}\right)=\frac{-2 A_{0}}{1-4 a}$. This solution can be formulated as

$$
y(t)=e^{\sqrt{a} i t} \phi(t)+e^{-\sqrt{a} i t} \phi^{*}(t)+\mathcal{O}\left(\epsilon^{2}\right)
$$

where

$$
\phi(t)=A_{0}+\epsilon\left(A_{1}+A_{0}\left(\frac{1}{1+2 \sqrt{a}} e^{i t}+\frac{1}{1-2 \sqrt{a}} e^{-i t}\right)\right) .
$$

Up to order of $\epsilon$, the solutions to the Mathieu equation are stable provided $a>0$ and $a \neq \frac{n^{2}}{4}, n>0$, since in this case the real part of the exponent of $e^{i \sqrt{a} t}$ is zero (see the remark 6.24).

The Floquet theorem and remark 6.23 ensure that if we continue the same procedure, we will get the following form for the solution of the Mathieu equation:

$$
y(t)=e^{\sqrt{a} i t} \phi(t)+e^{-\sqrt{a} i t} \phi^{*}(t),
$$

where $\phi(t)$ is a periodic function.

CASE II) $a$ in a small neighborhood of $\frac{1}{4}$.

In this case we must have at least one secular term in the first order correction. But here we will seek something more. Not only do we want to find the solutions to the Mathieu equation when $a=\frac{1}{4}$, but we want to find stability boundaries near $a=\frac{1}{4}$. To do so, we make the Ansatz

$$
a=\frac{1}{4}+a_{1} \epsilon+a_{2} \epsilon^{2}+\ldots
$$




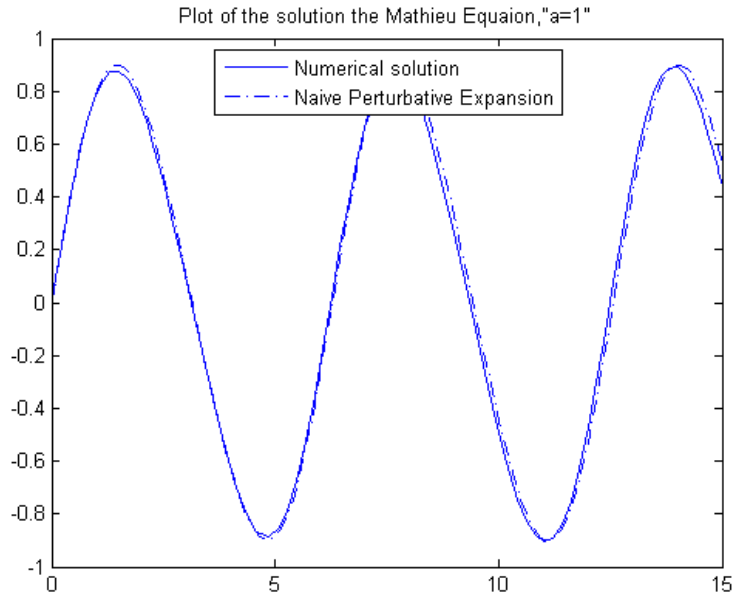

Figure 2.3: A plot of the solution to the Mathieu equation when $a=1$ and $\epsilon=.2$. Note that if we are looking for the second order approximation, $a$ cannot be 1 ; but because we are investigating the first order $\mathrm{RG}$, we only have the limitation $a \neq \frac{1}{4}$.

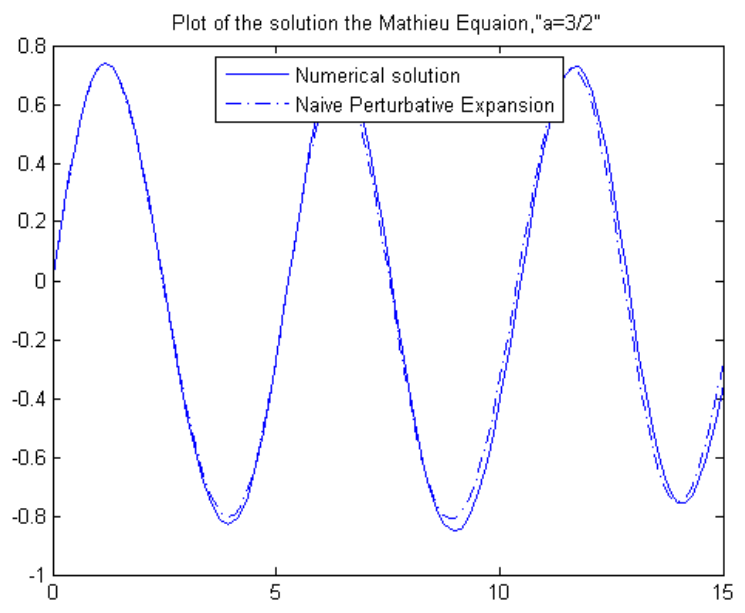

Figure 2.4: A plot of the solution to the Mathieu equation when $a=\frac{3}{2}$, and $\epsilon=.2$. Note that there does not exist any natural number $n$ such that $\frac{3}{2}=\frac{n^{2}}{4}$ 
Notice that if we let $a_{i}=0$, for $i \geq 1$, the solution formally reduces to the case $a=\frac{1}{4}$. Also note that whether a solution is stable or unstable will in general depend on $a_{i}$ s.

By the above assumption, the Mathieu equation reads

$$
\ddot{y}+\left(\frac{1}{4}+\left(2+a_{1}\right) \epsilon \cos t\right) y=0 .
$$

The naive expansion will end up with a secular term which contains $\epsilon\left(t-t_{0}\right)$. To renormalize the secular term by multiple scale analysis, we define $\tau:=\epsilon t$. Now suppose the new expansion

$$
y(t)=Y_{0}(t, \tau)+\epsilon Y_{1}(t, \tau)+\ldots .
$$

To renormalize the secular terms, we insert expansions (2.43) and (2.35) into (2.34). The result, up to and including $\mathcal{O}(\epsilon)$, is

$$
\begin{aligned}
& \frac{\partial^{2} Y_{0}}{\partial t^{2}}+\frac{Y_{0}}{4}=0 \\
& \frac{\partial^{2} Y_{1}}{\partial t^{2}}+\frac{Y_{1}}{4}=-\left(a_{1}+2 \cos t\right) Y_{0}-2 \frac{\partial^{2} Y_{0}}{\partial t \partial \tau} .
\end{aligned}
$$

The general solution to (2.36a) is

$$
Y_{0}=A(\tau) e^{\frac{i}{2} t}+A^{*}(\tau) e^{\frac{-i}{2} t} .
$$

Similar to the previous example, we try to absorb the secularity by restricting the integral constant $A$. To find the restriction, we substitute (2.37) into (2.36b)

$$
\frac{\partial^{2} Y_{1}}{\partial t^{2}}+\frac{Y_{1}}{4}=-a_{1} A e^{\frac{i}{2} t}-A e^{\frac{3 i}{2} t}-A^{*} e^{\frac{i}{2} t}-i A^{\prime} e^{\frac{i}{2} t}+c . c .,
$$

where "prime" stands for $\frac{d}{d \tau}$.

Since $e^{ \pm \frac{i}{2} t}$ are solutions to the homogeneous part, the first order correction will have secularities unless the coefficients of $e^{ \pm \frac{i}{2} t}$, on the right hand side of (2.38), are zero

$$
a_{1} A(\tau)+i A^{\prime}(\tau)+A^{*}(\tau)=0 .
$$


Note that we obtain two equations for $A$ and $A^{*}$ which are conjugate of one another. Then we need to solve one of them.

To solve (2.39) we let $A(\tau)=B(\tau)+i C(\tau)$. Then we find the system

$$
\left\{\begin{array}{l}
B^{\prime}=\left(1+a_{1}\right) C \\
C^{\prime}=\left(1-a_{1}\right) B
\end{array}\right.
$$

The solution to this system of equations reads

$$
\begin{aligned}
& B=d_{1} \sqrt{1-a_{1}} e^{\left.\sqrt{1-a_{1}^{2}} \epsilon\right) t}-d_{2} \sqrt{1-a_{1}} e^{-\sqrt{1-a_{1}^{2}} \epsilon t} \\
& C=d_{1} \sqrt{1+a_{1}} e^{\sqrt{1-a_{1}^{2}} \epsilon t}+d_{2} \sqrt{1+a_{1}} e^{-\sqrt{1-a_{1}^{2}} \epsilon t}
\end{aligned}
$$

for some constants $d_{1}$ and $d_{2}$. Therefore

$$
y(t)=\left(d_{1}\left(\sqrt{1-a_{1}}+i \sqrt{1+a_{1}}\right) e^{\sqrt{1-a_{1}^{2}} \epsilon t}+d_{2}\left(\sqrt{1-a_{1}}-i \sqrt{1+a_{1}}\right) e^{-\sqrt{1-a_{1}^{2}} \epsilon t}\right) e^{\frac{i}{2} t}+c . c .
$$

According to the remark 6.24 , solutions are unstable when $\left(1-a_{1}^{2}\right)$ is positive and stable otherwise. Therefore, instability occurs when $\left|a_{1}\right|<1$ and stability occurs when $\left|a_{1}\right|>1$. Hence, for small values of $\epsilon, a=\frac{1}{4} \pm \epsilon+\ldots$ are stability boundary lines.

\subsubsection{The RG Method Applied to the Mathieu Equation}

In this subsection, we apply the RG method to Mathieu's equation and show that the results are the same as the ones obtained from the multiple scale analysis, (see [12]). So, the results of this subsection will not be new, but will show that the multiple scale analysis and RG method are equivalent in the case of Mathieu's equation.

It is worth noting that, for the cases $a \neq \frac{n^{2}}{4}$, the naive perturbative expansion does not have any secular term(s). So the naive perturbative expansion works and does not need to be renormalized [see the figure (2.3) and (2.4) to observe how well the first order perturbative expansion approximates the solutions.] If $a=\frac{n^{2}}{4}, n>0$, 


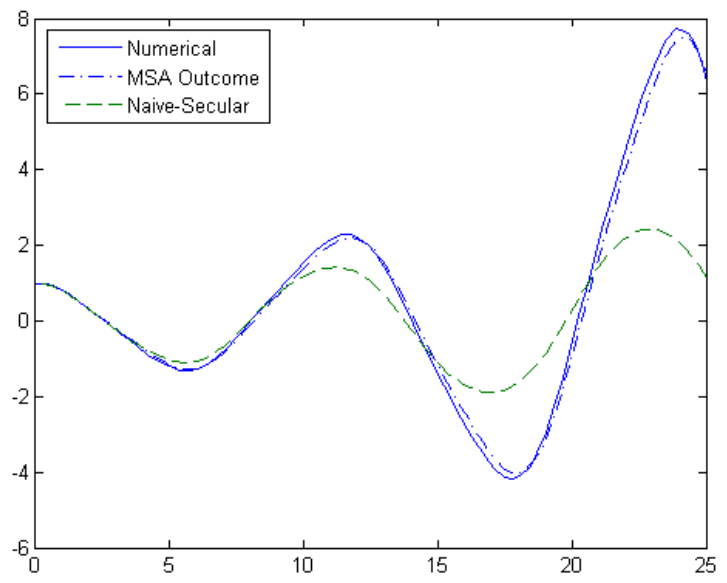

Figure 2.5: A plot of the multiple scale analysis (MSA) outcome applied to the Mathieu equation for the values $a=\frac{1}{4}$ and $\epsilon=.1$.

we need to renormalize the secular term(s) to have a valid approximation. This is the case to which RG can be applied to renormalize the secular term(s). As in the multiple scale analysis above, we let $a=\frac{1}{4}$.

Recall the Mathieu equation

$$
\ddot{y}+(a+2 \epsilon \cos t) y=0
$$

where $a$ is a positive parameter. We again try a naive perturbative expansion for the solution to (2.42):

$$
y(t)=y_{0}(t)+\epsilon y_{1}(t)+\mathcal{O}\left(\epsilon^{2}\right)
$$

Also to find stability boundaries, we postulate $a$ as a function of $\epsilon$ so that

$$
a=\frac{1}{4}+a_{1} \epsilon+\ldots
$$

Then we get again

$$
\begin{aligned}
& \ddot{y}_{0}(t)+\frac{1}{4} y_{0}(t)=0, \\
& \ddot{y}_{1}(t)+\frac{1}{4} y_{1}(t)=-\left(a_{1}+2 \cos t\right) y_{0}(t),
\end{aligned}
$$




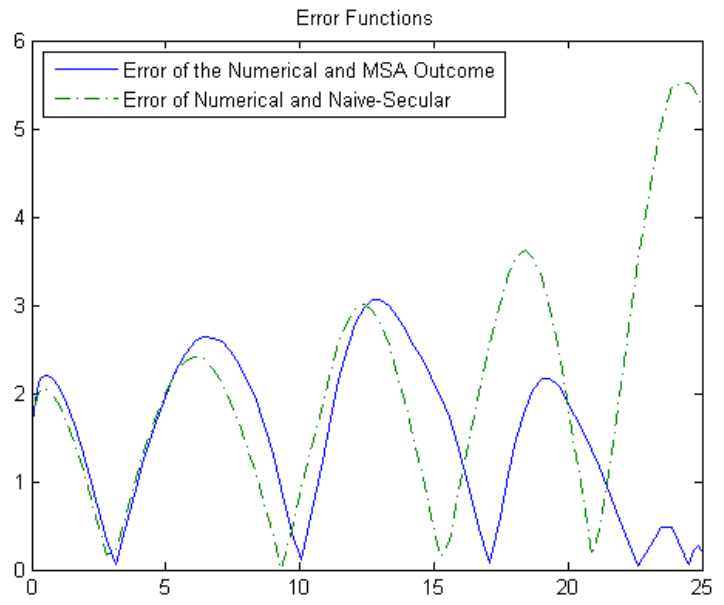

Figure 2.6: Plots of relative errors between the numerical result and the naive perturbative expansion which contains secular term and the multiple scale analysis (or equivalently RG) outcome which has absorbed the secularities. The values of parameters are $a=1$ and $\epsilon=.2$.

and so on.

By solving the equations (2.44), we obtain

$$
\begin{aligned}
y(t)=R_{0} \cos \left(\frac{t}{2}+\theta_{0}\right)+\epsilon R_{0} & \left(-\frac{1}{2} \cos \left(\frac{t}{2}+\theta_{0}\right)+\frac{1}{2} \cos \left(\frac{3 t}{2}+\theta_{0}\right)-\right. \\
& \left.a_{1}\left(t-t_{0}\right) \sin \left(\frac{t}{2}+\theta_{0}\right)-\left(t-t_{0}\right) \sin \left(\frac{t}{2}-\theta_{0}\right)\right)+\mathcal{O}\left(\epsilon^{2}\right),
\end{aligned}
$$

where $R_{0}$ and $\theta_{0}$ are integral constants that can be determined by initial conditions.

Now we introduce a new parameter, $\sigma$, between $t_{0}$ and $t$ and then split $\left(\sigma-t_{0}\right)+$ $(t-\sigma):$

$$
\begin{aligned}
y(t)= & R_{0} \cos \left(\frac{t}{2}+\theta_{0}\right)+\epsilon R_{0}\left(-\frac{1}{2} \cos \left(\frac{t}{2}+\theta_{0}\right)+\frac{1}{2} \cos \left(\frac{3 t}{2}+\theta_{0}\right)-\right. \\
a_{1}\left(\sigma-t_{0}\right) \sin \left(\frac{t}{2}+\theta_{0}\right)-\left(\sigma-t_{0}\right) \sin \left(\frac{t}{2}-\theta_{0}\right)- & a_{1}(t-\sigma) \sin \left(\frac{t}{2}+\theta_{0}\right)- \\
& \left.(t-\sigma) \sin \left(\frac{t}{2}-\theta_{0}\right)\right)+\mathcal{O}\left(\epsilon^{2}\right) .
\end{aligned}
$$


Hence

$$
\begin{gathered}
y(t)=R_{0} \cos \left(\frac{t}{2}+\theta_{0}\right)+\epsilon R_{0}\left(\left(-\frac{1}{2}+\left(\sigma-t_{0}\right) \sin 2 \theta_{0}\right) \cos \left(\frac{t}{2}+\theta_{0}\right)-\right. \\
\left(a_{1}+\cos 2 \theta_{0}\right)\left(\sigma-t_{0}\right) \sin \left(\frac{t}{2}+\theta_{0}\right)+\frac{1}{2} \cos \left(\frac{3 t}{2}+\theta_{0}\right)- \\
\left.\left(a_{1}+\cos 2 \theta_{0}\right)(t-\sigma) \sin \left(\frac{t}{2}+\theta_{0}\right)+(t-\sigma) \sin 2 \theta_{0} \cos \left(\frac{t}{2}+\theta_{0}\right)\right)+\mathcal{O}\left(\epsilon^{2}\right) .
\end{gathered}
$$

Now we use a multiplicative and an additive renormalization constants

$$
\begin{aligned}
R_{0}\left(t_{0}\right) & =R(\sigma)+\epsilon a\left(\sigma, t_{0}\right) R(\sigma)+\mathcal{O}\left(\epsilon^{2}\right), \\
\theta_{0}\left(t_{0}\right) & =\theta(\sigma)+\epsilon b\left(\sigma, t_{0}\right)+\mathcal{O}\left(\epsilon^{2}\right) .
\end{aligned}
$$

to absorb higher order integral constants as well as terms which contain the factor $\sigma-t_{0}$

Choosing

$$
\begin{aligned}
& a=\frac{1}{2}-\left(\sigma-t_{0}\right) \sin 2 \theta, \\
& b=-\left(\sigma-t_{0}\right)\left(a_{1}+\cos 2 \theta\right),
\end{aligned}
$$

gives

$$
\begin{aligned}
y(t)=R \cos \left(\frac{t}{2}+\theta\right)+\epsilon R\left((t-\sigma) \sin 2 \theta \cos \left(\frac{t}{2}+\theta\right)+\frac{1}{2} \cos \left(\frac{3 t}{2}+\theta\right)-\right. \\
\left.\left(a_{1}+\cos 2 \theta\right)(t-\sigma) \sin \left(\frac{t}{2}+\theta\right)\right)+\mathcal{O}\left(\epsilon^{2}\right),
\end{aligned}
$$

where $R=R(\sigma)$ and $\theta=\theta(\sigma)$.

By applying RG condition, $\frac{\partial y}{\partial \sigma}=\mathcal{O}\left(\epsilon^{2}\right)$, we find

$$
\begin{gathered}
\frac{d R}{d \sigma} \cos \left(\frac{t}{2}+\theta\right)-R \frac{d \theta}{d \sigma} \sin \left(\frac{t}{2}+\theta\right)+\epsilon \frac{d R}{d \sigma}\left((t-\sigma) \sin 2 \theta \cos \left(\frac{t}{2}+\theta\right)+\frac{1}{2} \cos \left(\frac{3 t}{2}+\theta\right)-\right. \\
\left.\left(a_{1}+\cos 2 \theta\right)(t-\sigma) \sin \left(\frac{t}{2}+\theta\right)\right)+\epsilon R\left(-\sin 2 \theta \cos \left(\frac{t}{2}+\theta\right)+2 \frac{d \theta}{d \sigma}(t-\sigma) \cos 2 \theta \cos \left(\frac{t}{2}+\theta\right)-\right. \\
(t-\sigma) \frac{d \theta}{d \sigma} \sin 2 \theta \cos \left(\frac{t}{2}+\theta\right)-\frac{1}{2} \frac{d \theta}{d \sigma} \sin \left(\frac{3 t}{2}+\theta\right)-2 \frac{d \theta}{d \sigma} \sin 2 \theta(t-\sigma) \sin \left(\frac{t}{2}+\theta\right)- \\
\left.\left(a_{1}+\cos 2 \theta\right) \sin \left(\frac{t}{2}+\theta\right)+\left(a_{1}+\cos 2 \theta\right) \frac{d \theta}{d \sigma} \cos \left(\frac{t}{2}+\theta\right)\right)=\mathcal{O}\left(\epsilon^{2}\right) .
\end{gathered}
$$


Thus $\frac{d R}{d \sigma} \cos \left(\frac{t}{2}+\theta\right)-R \frac{d \theta}{d \sigma} \sin \left(\frac{t}{2}+\theta\right)=\mathcal{O}(\epsilon)$. This implies

$$
\begin{aligned}
\frac{d R}{d \sigma} & =\mathcal{O}(\epsilon), \\
\frac{d \theta}{d \sigma} & =\mathcal{O}(\epsilon) .
\end{aligned}
$$

By substituting (2.46) into (2.45), $\mathcal{O}\left(\epsilon^{2}\right)$ absorbs terms of order of $\epsilon^{2}$. Hence

$$
\begin{aligned}
\frac{d R}{d \sigma} \cos \left(\frac{t}{2}+\theta\right)-R \frac{d \theta}{d \sigma} \sin \left(\frac{t}{2}+\theta\right)-\epsilon R\left(\sin 2 \theta \cos \left(\frac{t}{2}+\theta\right)+\right. & \left.\left(a_{1}+\cos 2 \theta\right) \sin \left(\frac{t}{2}+\theta\right)\right)=\mathcal{O}\left(\epsilon^{2}\right),
\end{aligned}
$$

Consequently, the amplitude and phase equations (RG equations) read as

$$
\begin{aligned}
& \frac{d R}{d \sigma}=\epsilon R \sin 2 \theta+\mathcal{O}\left(\epsilon^{2}\right) \\
& \frac{d \theta}{d \sigma}=\epsilon\left(a_{1}+\cos 2 \theta\right)+\mathcal{O}\left(\epsilon^{2}\right) .
\end{aligned}
$$

To solve (2.47), we use the cartesian coordinates: $R e^{i \theta}=B+i C$. Hence

$$
\begin{aligned}
& B^{\prime}(\sigma)=\frac{d R}{d \sigma} \cos \theta-\frac{d \theta}{d \sigma} R \sin \theta+\mathcal{O}\left(\epsilon^{2}\right)=\epsilon R \sin 2 \theta \cos \theta- \\
& \epsilon R\left(a_{1}+\cos 2 \theta\right) \sin \theta+\mathcal{O}\left(\epsilon^{2}\right)=\epsilon\left(1-a_{1}\right) C(\sigma)+\mathcal{O}\left(\epsilon^{2}\right) .
\end{aligned}
$$

Note that we have used $\sin \theta=\sin 2 \theta \cos \theta-\cos 2 \theta \sin \theta$. Similarly, we have $C^{\prime}(\sigma)=$ $\epsilon\left(1+a_{1}\right) B(\sigma)+\mathcal{O}\left(\epsilon^{2}\right)$.

Thus the RG equations (with respect to $B$ and $C$ ) can be written as the system

$$
\left[\begin{array}{c}
\dot{B} \\
\dot{C}
\end{array}\right]=\epsilon\left[\begin{array}{cc}
0 & 1-a_{1} \\
1+a_{1} & 0
\end{array}\right]\left[\begin{array}{l}
B \\
C
\end{array}\right]+\mathcal{O}\left(\epsilon^{2}\right) .
$$

This implies that there is some $c$ such that

$$
\left\|\left[\begin{array}{c}
\dot{B} \\
\dot{C}
\end{array}\right]\right\|-\epsilon\left\|\left[\begin{array}{cc}
0 & 1-a_{1} \\
1+a_{1} & 0
\end{array}\right]\left[\begin{array}{l}
B \\
C
\end{array}\right]\right\| \leq c \epsilon^{2} .
$$


The solution to the system (2.48), up to the first order, is

$$
\left[\begin{array}{c}
\tilde{B} \\
\tilde{C}
\end{array}\right]=\left[\begin{array}{l}
d_{1} \sqrt{1-a_{1}} e^{\sqrt{1-a_{1}^{2}} \epsilon \sigma}-d_{2} \sqrt{1-a_{1}} e^{-\sqrt{1-a_{1}^{2}} \epsilon \sigma} \\
d_{1} \sqrt{1+a_{1}} e^{\sqrt{1-a_{1}^{2}} \epsilon \sigma}+d_{2} \sqrt{1+a_{1}} e^{-\sqrt{1-a_{1}^{2}} \epsilon \sigma}
\end{array}\right] .
$$

To find the error of approximation, let us define $\left[\begin{array}{l}W_{1} \\ W_{2}\end{array}\right]=\left[\begin{array}{l}B \\ C\end{array}\right]-\left[\begin{array}{l}\tilde{B} \\ \tilde{C}\end{array}\right]$. Thus

$$
\left\|\left[\begin{array}{l}
\dot{W}_{1} \\
\dot{W}_{2}
\end{array}\right]\right\|-\epsilon\left\|\left[\begin{array}{cc}
0 & 1-a_{1} \\
1+a_{1} & 0
\end{array}\right]\left[\begin{array}{l}
W_{1} \\
W_{2}
\end{array}\right]\right\| \leq c \epsilon^{2} .
$$

Hence

$$
\left\|\left[\begin{array}{l}
W_{1} \\
W_{2}
\end{array}\right]\right\| \leq c \epsilon^{2}\left\|e^{\epsilon A \sigma} \int_{0}^{\sigma} e^{\epsilon A \tau} d \tau\right\|=-c \epsilon\left\|A^{-1}\left(1-e^{\epsilon A \sigma}\right)\right\|=c \sigma \epsilon^{2}+\mathcal{O}\left(\sigma^{2} \epsilon^{3}\right),
$$

where

$$
A=\left[\begin{array}{cc}
0 & 1-a_{1} \\
1+a_{1} & 0
\end{array}\right]
$$

Thus $\left\|\left[\begin{array}{l}W_{1} \\ W_{2}\end{array}\right]\right\|=\mathcal{O}\left(\sigma \epsilon^{2}\right)$. Therefore

$$
\begin{aligned}
& B=d_{1} \sqrt{1-a_{1}} e^{\sqrt{1-a_{1}^{2}} \epsilon \sigma}-d_{2} \sqrt{1-a_{1}} e^{-\sqrt{1-a_{1}^{2}} \epsilon \sigma}+\mathcal{O}\left(\sigma \epsilon^{2}\right), \\
& C=d_{1} \sqrt{1+a_{1}} e^{\sqrt{1-a_{1}^{2}} \epsilon \sigma}+d_{2} \sqrt{1+a_{1}} e^{-\sqrt{1-a_{1}^{2}} \epsilon \sigma}+\mathcal{O}\left(\sigma \epsilon^{2}\right),
\end{aligned}
$$

for some constants $d_{1}$ and $d_{2}$. Now setting $\sigma=t$ we find

$$
y(t)=\left(d_{1}\left(\sqrt{1-a_{1}}+i \sqrt{1+a_{1}}\right) e^{\sqrt{1-a_{1}^{2}} \epsilon t}+d_{2}\left(\sqrt{1-a_{1}}-i \sqrt{1+a_{1}}\right) e^{-\sqrt{1-a_{1}^{2}} \epsilon t}\right) e^{\frac{i}{2} t}+\text { c.c. }
$$

\subsection{A Simple Second Order ODE}

In this section we are going to find approximate solutions to a boundary value problem

$$
\epsilon y^{\prime \prime}(x)+y^{\prime}(x)+y(x)=0, \quad \epsilon \ll 1, y(0)=0, y(1)=1
$$


This equation is a second order linear ordinary differential equation with constant coefficients whose solution decays exponentially to an equilibrium without oscillations.

We first will find the exact solution using the elementary methods. Then we will apply the boundary layer theory and RG method to this equation to find the zerothorder approximations. At the end of this section we will compare the results of the boundary layer theory and RG method with the numerical results.

This is a very simple ODE which can be solved by substituting $y(x)=e^{r x}$. Thus the auxiliary equation reads $\epsilon r^{2}+r+1=0$ whose solutions are

$$
r_{1}, r_{2}=\frac{1}{2 \epsilon}(1 \pm \sqrt{1-4 \epsilon}) .
$$

Hence the solution to $(2.50)$ is

$$
y(x)=\frac{e^{\frac{1}{2 \epsilon}(1+\sqrt{1-4 \epsilon}) x}-e^{\frac{1}{2 \epsilon}(1-\sqrt{1-4 \epsilon}) x}}{e^{\frac{1}{2 \epsilon}(1+\sqrt{1-4 \epsilon})}-e^{\frac{1}{2 \epsilon}(1-\sqrt{1-4 \epsilon})}} .
$$

For $\epsilon$ small enough, the solution can be approximated by

$$
y(x) \sim \frac{e^{\frac{x}{\epsilon}}-1}{e^{\frac{1}{\epsilon}}-1} .
$$

We observe, on the one hand, that $y(x)$ approaches to 1 in the region $\epsilon \ll x \leq 1$, and on the other hand, it is close to 0 when $x \sim \epsilon^{\alpha}, \alpha \geq 1$. This causes an abrupt change in an $\epsilon$-neighborhood of the origin. Such region, in which the solution has an abrupt change, is called a boundary layer region.

Instead of this exact solution, we may approximate solutions to (2.50). To do this, we write the Taylor expansion representation of $(1-a)^{\frac{1}{2}}=1-\frac{1}{2} a+\mathcal{O}\left(a^{2}\right)$ to approximate values of $r_{1}$ and $r_{2}$. Hence an approximation for (2.50) is

$$
y(x)=\frac{e^{-x}-e^{x-\frac{x}{\epsilon}}}{e^{-1}-e^{1-\frac{1}{\epsilon}}}+\mathcal{O}\left(\epsilon^{2}\right) .
$$

\subsubsection{The Boundary Layer Theory}

Now we apply the Boundary Layer method to find an approximate solution to the boundary value problem (2.50). (For a more general case see [3] chapter 9.) 
The highest derivative term, in (2.50), is multiplied by a small parameter, the solution may have the boundary layer structure; because when we let $\epsilon=0$, the solution loses the highest derivative term's influence on the behavior of the leading term, which is expected to have the main contribution for the approximation. To find out whether this equation has a boundary layer structure or not, we try the naive perturbative expansion:

$$
y(x)=\sum_{n=0}^{\infty} y_{n}(x) \epsilon^{n} .
$$

If this naive expansion properly satisfies the boundary values, the equation is not singular. Consequently, it does not have any boundary layer structure. For more details about the boundary layer theory see the Appendix, definitions 6.10 to 6.11.

By substituting (2.51) into (2.50) we get

$$
\begin{gathered}
y_{0}^{\prime}(x)+y_{0}(x)=0, \quad y_{0}(0)=0, y_{0}(1)=1, \\
y_{1}^{\prime}(x)+y_{1}(x)=-y_{0}^{\prime \prime}(x), \quad y_{1}(0)=0, y_{1}(1)=0, \\
\vdots \\
y_{n}^{\prime}(x)+y_{n}(x)=-y_{n-1}^{\prime \prime}(x), \quad y_{n}(0)=0, y_{n}(1)=0,
\end{gathered}
$$

and so forth. To find the boundary layer region, if any, we only need to find the leading term (i.e. $\epsilon=0$ ). We have

$$
y_{0}(x)=c e^{-x}
$$

We observe that, only the second boundary condition is consistent with $y_{0}(x) \not \equiv 0$. Then $y_{0}(x)=e^{1-x}$.

Since $y_{0}(x)=e^{1-x}$ does not satisfy the boundary condition at $x=0$, it is not valid everywhere! Then there must be an abrupt change near $x=0$. Technically, the solution in this region is called the inner solution, and usually is represented by $y_{i n}$. The region in which the solution has a slowly varying behavior, outside of any boundary layer region, is called the outer region. The solution in this region is called 
the outer solution and represented by $y_{\text {out }}$. Note that $y_{0}(x)=e^{1-x}$ is the leading term of the outer solution.

Now to find the thickness of the boundary layer, say $\delta$, we let $\delta X=x$. By substituting this into (2.50) we get

$$
\frac{\epsilon}{\delta^{2}} y^{\prime \prime}+\frac{1}{\delta} y^{\prime}+y=0, \quad \epsilon \ll 1,
$$

where $y^{\prime}=\frac{d y}{d X}$.

We determine $\delta$ under the following assumptions:

- The highest derivative term provides the major contribution to $y_{i n}$.

- When $y_{\text {in }}$ has an abrupt change in the boundary layer region and, therefore, has other than constant or linear behavior in this region.

Let $\delta=\epsilon^{\alpha}, \alpha \geq 0$. Substituting this into (2.52) gives

$$
\epsilon^{1-2 \alpha} y^{\prime \prime}(x)+\epsilon^{-\alpha} y^{\prime}(x)+y(x)=0 .
$$

We have 3 possibilities:

- $1-2 \alpha=0$. In this case, not only do we lose the contribution of the highest derivative term, but the behavior $y_{\text {in }}$ is linear. Hence this case is inconsistent with our assumptions.

- $-\alpha=0$ which implies that the thickness of the inner region is 1 and the leading term of $y_{\text {in }}$ has a constant or linear behavior. Then this case is also inconsistent with our assumption.

- $1-2 \alpha=-\alpha$, which implies $\alpha=1$. This case is the only consistent case. Then the inner region's thickness is $\epsilon$.

Now to find the inner solution, we use the change of variable $X=\frac{x}{\epsilon}$. Because the thickness of the boundary layer is $\epsilon$, by this change of variable, indeed, we are 
"zooming in" the boundary layer region. More precisely, the independent variable no longer takes small values in the inner region.

Let $Y(X)=y(x)$. Thus

$$
Y^{\prime \prime}+Y^{\prime}+\epsilon Y=0,
$$

where ' stands for $\frac{d}{d X}$.

We postulate a naive perturbative expansion for the inner solution: $Y_{i n}=Y_{0}+$ $\epsilon Y_{1}+\ldots$. But because we are only interested in finding the leading term of $Y_{i n}$, we let $\epsilon=0$. Hence

$$
Y_{0}=c e^{-X}+d, Y_{0}(0)=0 .
$$

Therefore

$$
Y_{0}=c e^{-X}-c .
$$

The constant $c$ can be determined by matching $Y_{0}$ and $y_{0}$ in the overlap of the inner and outer regions. Such region is called the intermediate or overlap region. In this region $x \rightarrow 0, X=\frac{x}{\epsilon} \rightarrow \infty$ and $\epsilon \rightarrow 0$. By these, we have $y_{0}=e$ and $Y_{0}=-c$. Hence

$$
Y_{0}(X)=e-e^{1-X} .
$$

In the boundary layer theory, to find a uniform approximation which is valid throughout the region of integration, we use the formula

$$
y_{\text {unif }}=Y_{\text {in }}+y_{\text {out }}-y_{\text {match }},
$$

where $y_{\text {match }}$ is the solution in the overlap region.

Since here we are only interested in the leading order, then we use the following formula

$$
y_{\text {unif }, 0}=Y_{0}+y_{0}-y_{\text {match }, 0},
$$

which turns out

$$
y_{u n i f, 0}=e^{1-x}-e^{1-\frac{x}{\epsilon}} .
$$


See the figure (2.7) to observe how precisely (2.53) approximates well the exact solution.

Remark 2.2. Sometimes using boundary conditions may fail to indicate if there exists any boundary layer region. Then we need more analysis to find the location of the boundary layer. This is one of the impediments and disadvantages of using the boundary layer theory.

Remark 2.3. If the boundary layer is located at $x=a$, we have $|x-a| \gg \mathcal{O}(\delta)$ in the outer region and $|x-a| \leq \mathcal{O}(\delta)$ in the inner region, as $\epsilon \rightarrow 0$, where $\delta$ is the thickness of the boundary layer. In particular, when $a=0$, the outer region is $|x| \gg \mathcal{O}(\delta)$ and the inner region is $|x| \leq \mathcal{O}(\delta)$.

Remark 2.4. Although the Boundary Layer theory seems to be straightforward in this example, this method might be very complicated to be applied to a slightly different boundary value problem. (See [3] for more examples of boundary layer theory.)

\subsubsection{The RG Method Applied to a Simple Second Order ODE}

We are going to apply the RG method to the boundary value problem

$$
\epsilon y^{\prime \prime}(x)+y^{\prime}(x)+y(x)=0, \quad \epsilon \ll 1, y(0)=0, y(1)=1
$$

See $[12]$.

Using the change of variable $x=\epsilon t$, we obtain

$$
Y^{\prime \prime}(t)+Y^{\prime}(t)+\epsilon Y(t)=0, \quad \forall t \in\left[0, \frac{1}{\epsilon}\right], Y(t)=y(x) .
$$

Then the problem is converted to finding an approximation to the solution of (2.55) using the RG method. Let us assume

$$
Y(t)=Y_{0}(t)+\epsilon Y_{1}(t)+\mathcal{O}\left(\epsilon^{2}\right)
$$


Then by inserting (2.56) into (2.55), we get

$$
\begin{aligned}
& Y_{0}^{\prime \prime}(t)+Y_{0}^{\prime}(t)=0 \\
& Y_{1}^{\prime \prime}(t)+Y_{1}^{\prime}(t)=-Y_{0}(t)
\end{aligned}
$$

and so on. The general solutions to $(2.57 \mathrm{a})$ and $(2.57 \mathrm{~b})$ can be written:

$$
\begin{aligned}
& Y_{0}=A_{0} e^{-t}+B_{0}, \\
& Y_{1}=A_{0} A_{1} e^{-t}+A_{0}\left(t-t_{0}\right) e^{-t}-B_{0}\left(t-t_{0}\right)+B_{0} B_{1} .
\end{aligned}
$$

Inserting (2.58) into (2.56) gives

$$
Y(t)=A_{0} e^{-t}+B_{0}+\epsilon\left(A_{0} A_{1} e^{-t}+B_{0} B_{1}+A_{0}\left(t-t_{0}\right) e^{-t}-B_{0}\left(t-t_{0}\right)\right)+\mathcal{O}\left(\epsilon^{2}\right) .
$$

Here, one may say because we have a boundary value problem, the independent variable cannot grow and then the factor $\left(t-t_{0}\right)$ does not break down the convergence of the naive expansion. This is not true, because the independent variable in (2.56) is $t$ which can tend to infinity as $\epsilon$ tends to zero and $x \in[0,1]$. So, we introduce the new parameter, $\sigma$ :

$$
\begin{aligned}
Y(t)=A_{0} e^{-t}+B_{0}+\epsilon\left(A_{0} A_{1} e^{-t}+\right. & B_{0} B_{1}+A_{0}\left(\sigma-t_{0}\right) e^{-t}- \\
& \left.B_{0}\left(\sigma-t_{0}\right)+A_{0}(t-\sigma) e^{-t}-B_{0}(t-\sigma)\right)+\mathcal{O}\left(\epsilon^{2}\right)
\end{aligned}
$$

Now we define multiplicative renormalization constants:

$$
\begin{aligned}
& A_{0}=A(\sigma)+\epsilon a A(\sigma)+\mathcal{O}\left(\epsilon^{2}\right), \\
& B_{0}=B(\sigma)+\epsilon b B(\sigma)+\mathcal{O}\left(\epsilon^{2}\right) .
\end{aligned}
$$

We choose $a$ and $b$ so as to absorb the terms with the multiplier $\left(\sigma-t_{0}\right)$ as well as a solution to the homogeneous part of $(2.57 \mathrm{~b})$ (appearing in $Y_{1}$ ):

$$
\begin{aligned}
& a=-A_{1}-\left(\sigma-t_{0}\right), \\
& b=-B_{1}+\left(\sigma-t_{0}\right) .
\end{aligned}
$$


Therefore

$$
Y(t)=A e^{-t}+B+\epsilon\left(A(t-\sigma) e^{-t}-B(t-\sigma)\right)+\mathcal{O}\left(\epsilon^{2}\right)
$$

where $A=A(\sigma)$.

By applying RG condition $\frac{\partial Y}{\partial \sigma}=\mathcal{O}\left(\epsilon^{2}\right)$, we obtain

$$
\frac{d A}{d \sigma} e^{-t}+\frac{d B}{d \sigma}+\epsilon\left(\frac{d A}{d \sigma}(t-\sigma) e^{-t}-A e^{-t}-\frac{d B}{d \sigma}(t-\sigma)+B\right)=\mathcal{O}\left(\epsilon^{2}\right) .
$$

The independence of $e^{-t}$ and 1 implies that $\frac{d A}{d \sigma}=\mathcal{O}(\epsilon)$ and $\frac{d B}{d \sigma}=\mathcal{O}(\epsilon)$. Thus

$$
\frac{d A}{d \sigma} e^{-t}+\frac{d B}{d \sigma}-A \epsilon e^{-t}+\epsilon B=\mathcal{O}\left(\epsilon^{2}\right)
$$

Again, we use the independence of $e^{-t}$ and 1 to obtain $\mathrm{RG}$ equations

$$
\begin{aligned}
& \frac{d A}{d \sigma}-A \epsilon=\mathcal{O}\left(\epsilon^{2}\right), \\
& \frac{d B}{d \sigma}+B \epsilon=\mathcal{O}\left(\epsilon^{2}\right) .
\end{aligned}
$$

Hence by integration and applying Gronwall's lemma 6.27 (like what was done in the Rayleigh Oscillator and the Mathieu equation) we find

$$
\begin{aligned}
& A(\sigma)=c_{1} e^{\epsilon \sigma}+\mathcal{O}\left(\epsilon\left(e^{\epsilon t}-1\right)\right), \\
& B(\sigma)=c_{2} e^{-\epsilon \sigma}+\mathcal{O}\left(\epsilon\left(1-e^{-\epsilon t}\right)\right) .
\end{aligned}
$$

Setting $\sigma=t$ and substituting (2.60) into (2.59) gives the solution to (2.55)

$$
Y(t)=c_{1} e^{\epsilon t-t}+c_{2} e^{-\epsilon t}+\mathcal{O}\left(\epsilon e^{\epsilon t}\right)
$$

Going back to the original variable we obtain

$$
y(x)=c_{1} e^{x-\frac{x}{\epsilon}}+c_{2} e^{-x}+\mathcal{O}\left(\epsilon e^{x}\right)
$$

By applying the boundary values we get

$$
y(x)=\frac{1}{e^{-1}-e^{1-\frac{1}{\epsilon}}}\left(e^{-x}-e^{x-\frac{x}{\epsilon}}\right)+\mathcal{O}\left(\epsilon e^{x}\right) .
$$

To compare the results of RG method and the boundary layer theory to the exact solution, see the figure (2.7). 


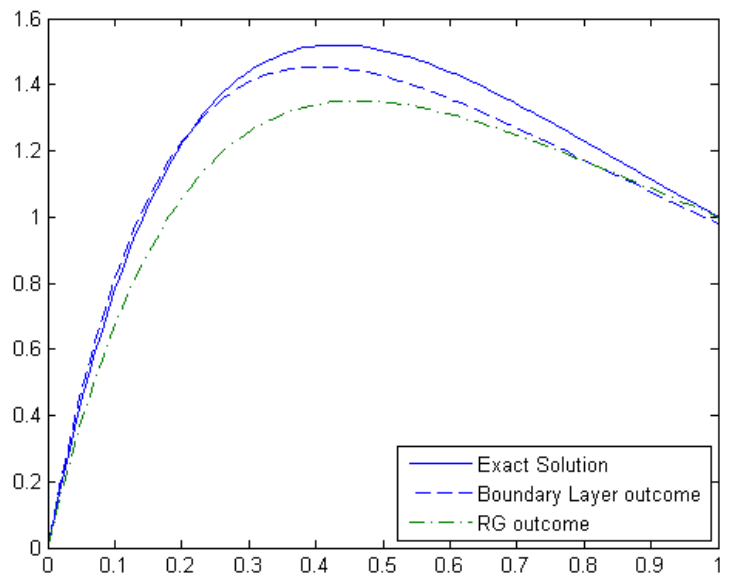

Figure 2.7: A plot of the solution to BVP (2.54), when $\epsilon=0.2$. We observe that the maximum error of the RG outcome is .2 which ensures us that the error we have gained in (2.61) is true.

\subsection{The Time-Independent Schrödinger Equation}

A second-order homogeneous linear differential equation is in Schrödinger form if $y^{\prime}$ term is absent. In this subsection we would like to approximate the solutions to the time-independent Schrödinger equation,

$$
\epsilon^{2} y^{\prime \prime}(x)=Q(x) y(x)
$$

The solutions to (2.62) can be studied in two different cases, whether $Q(x)$ has any root(s) or not. Then, to approximate the solutions, we have to separate the problem into two cases:

- $Q(x)$ is absolutely positive or negative throughout the real line.

- $Q(x)$ has roots.

For the second case, we consider the easiest case when $Q(x)$ has only one zero at $x=0$. Technically, the equation is said to have a turning point at $x=0$. 
We will approximate the solutions using Wentzel-Kramers-Brillouin (WKB [8, 9, 10]) theory and the RG method. We, also, will observe that the RG method and the WKB theory are equivalent in the case of no turning point, and the approximations are close to each other in the other case (see the figure (2.9) and (2.10).)

\subsubsection{WKB Approximation Applied to the Time-Independent Schrödinger Equation}

We are going to apply the WKB approximation to the time-independent Schrödinger equation, see [3] chapter 10.

\section{CASE I)}

$Q(x)$ does not have any root and, without loss of generality, is a positive function. In WKB theory we make the Ansatz $y=e^{\frac{1}{\delta} \sum_{n=0}^{\infty} \delta^{n} S_{n}}$; where $S_{n}$ 's are smooth functions and $\delta \ll 1$. In this example, we only use the WKB-physical approximation: $y=e^{\frac{1}{\delta} S_{0}+S_{1}}$. (See WKB Theory section in the Appendix (6.5).) In order that this approximation is valid, we must have

$$
\begin{gathered}
\frac{1}{\delta} S_{0} \gg S_{1}, \\
\delta S_{2} \ll 1 .
\end{gathered}
$$

Taking derivative with respect to $x$ and substituting the result into (2.62) gives

$$
\frac{\epsilon^{2}}{\delta^{2}}\left(S_{0}^{\prime}\right)^{2}+\epsilon^{2}\left(S_{1}^{\prime}\right)^{2}+\frac{2 \epsilon^{2}}{\delta} S_{0}^{\prime} S_{1}^{\prime}+\frac{\epsilon^{2}}{\delta} S_{0}^{\prime \prime}+\epsilon^{2} S_{1}^{\prime \prime}=Q(x)
$$

Since $\delta$ is a small parameter and $\frac{1}{\delta} S_{0} \gg S_{1}$, the following natural asymptotic relations hold

$$
\begin{aligned}
\epsilon^{2} S_{1}^{\prime \prime} & \ll \frac{\epsilon^{2}}{\delta} S_{0}^{\prime \prime}, \\
\epsilon^{2}\left(S_{1}^{\prime}\right)^{2} & \ll \frac{\epsilon^{2}}{\delta} S_{0}^{\prime} S_{1}^{\prime} .
\end{aligned}
$$

In addition, the largest term in the left hand side of $(2.63)$ is $\frac{\epsilon^{2}}{\delta^{2}}\left(S_{0}^{\prime}\right)^{2}$. Comparing its coefficient to the largest term in the right hand side, $Q(x)$, implies $\frac{\delta^{2}}{\epsilon^{2}} \sim 1$ or $\delta \sim \epsilon$. 
Then (2.63) reduces to

$$
\left(S_{0}^{\prime}\right)^{2}+\epsilon\left(S_{0}^{\prime \prime}+2 S_{0}^{\prime} S_{1}^{\prime}\right)=Q(x)
$$

Thus we obtain

$$
\begin{aligned}
& S_{0}(x)= \pm \int^{x} \sqrt{Q(t)} d t, \\
& S_{1}(x)=\frac{-1}{4} \ln Q(x)+c .
\end{aligned}
$$

Hence

$$
y(x)=c_{1} Q^{\frac{-1}{4}}(x) e^{\frac{1}{\epsilon} \int_{0}^{x} \sqrt{Q(t)} d t}+c_{2} Q^{\frac{-1}{4}}(x) e^{\frac{-1}{\epsilon} \int_{0}^{x} \sqrt{Q(t)} d t} .
$$

CASE II)

$Q(x)$ has one root at $x=0$ with the multiplicity 1 . Without loss of generality, let $Q(x)>0$ for $x>0$ and $Q(x)<0$ for $x<0$. Also suppose that

$$
|Q(x)| \gg x^{-2}, \quad|x| \rightarrow \infty
$$

and

$$
y(\infty)=0 .
$$

Since $Q$ is analytic near zero, by these assumptions, we have $Q(x) \sim a x$ for some $a>0$ and for $x$ in a small neighborhood of the origin.

The idea for approximating the solution is to divide the real axis into 3 distinct regions:

- Region I: where $x>0$ and $x \gg \epsilon^{\alpha}$ ( $\alpha$ is real). The solution in this region is represented by $y_{\mathrm{I}}$.

- Region II: where $|x| \ll 1$. The solution in this region is represented by $y_{\mathrm{II}}$.

- Region III: where $x<0$ and $(-x) \gg \epsilon^{\alpha}$. The solution in this region is represented by $y_{\mathrm{III}}$. 
We later will need to match $y_{\mathrm{I}}$ and $y_{\mathrm{II}}$ as well as $y_{\mathrm{II}}$ and $y_{\mathrm{III}}$ in overlap regions to have a valid global approximation.

In region I, $Q$ does not have any roots and we can use the formula we obtained in the CASE I:

$$
y_{\mathrm{I}}(x) \sim c_{1} Q^{\frac{-1}{4}}(x) e^{\frac{1}{\epsilon} \int_{0}^{x} \sqrt{Q(t)} d t}+c_{2} Q^{\frac{-1}{4}}(x) e^{\frac{-1}{\epsilon} \int_{0}^{x} \sqrt{Q(t)} d t}
$$

Based on the assumption (2.66) the boundary condition $y(\infty)=0$ is satisfied if $c_{1}=0$. Hence

$$
y_{\mathrm{I}}(x) \sim C Q^{\frac{-1}{4}}(x) e^{\frac{-1}{\epsilon} \int_{0}^{x} \sqrt{Q(t)} d t}
$$

We need to find the lower bound of the region in which (2.67) is a consistent approximation. Since $Q$ is analytic near the origin, we can write

$$
Q(x) \sim a x
$$

According to (6.31c) and (2.68), $S_{2}(x)= \pm \int^{x}\left(\frac{Q^{\prime \prime}}{8 Q^{\frac{3}{2}}}-\frac{5 Q^{\prime 2}}{32 Q^{\frac{5}{2}}}\right) \sim \pm \frac{5}{48} a^{\frac{-1}{2}} x^{\frac{-3}{2}}$. Now, according to $(6.32 \mathrm{~b})$, or , the lower bound for the validity of $y_{\mathrm{I}}$ is

$$
x \gg \epsilon^{\frac{2}{3}}
$$

Thus we can choose $\alpha=2 / 3$.

In the region II, we use a new variable, $t$, such that

$$
x=a^{-\frac{1}{3}} \epsilon^{\frac{2}{3}} t
$$

Notice that this change of variable is interpreted as a zooming in the narrow region near $x=0$. Thus $t \rightarrow \infty$ as $\epsilon \rightarrow 0 .^{1}$

Now we substitute (2.70) into (2.68) and (2.62) to obtain

$$
y_{\mathrm{II}}^{\prime \prime}=t y_{\mathrm{II}}
$$

\footnotetext{
${ }^{1}$ We used the same idea in the boundary layer problem.
} 
Equation (2.71) is a famous differential equation known as the Airy equation [3]. According to (6.2) in the Appendix, the general solution to the Airy equation is

$$
y_{\mathrm{II}}(x)=D \operatorname{Ai}(t)+E \operatorname{Bi}(t)=D \operatorname{Ai}\left(a^{\frac{1}{3}} \epsilon^{\frac{-2}{3}} x\right)+E \operatorname{Bi}\left(a^{\frac{1}{3}} \epsilon^{\frac{-2}{3}} x\right),
$$

where $\mathrm{Ai}$ and $\mathrm{Bi}$ are the Airy functions.

Since we assumed that $Q(x) \sim a x$, then the valid region is

$$
x \ll 1, \quad \epsilon \rightarrow 0+.
$$

To find the coefficients $D$ and $E$ we need to match $y_{\mathrm{I}}$ and $y_{\mathrm{II}}$ in the intermediate region, if any. $y_{\mathrm{I}}$ is valid in the region $x \gg \epsilon^{\frac{2}{3}}$ and $(2.72)$ is valid for $x \ll 1$. Since $Q(x) \sim a x$, for $\epsilon^{\frac{2}{3}} \ll x \ll 1$, therefore

$$
y_{\mathrm{I}}(x) \sim C a^{\frac{-1}{4}} x^{\frac{-1}{4}} e^{\frac{-2}{3 \epsilon} a^{\frac{1}{2}} x^{\frac{3}{2}}}, \quad x \rightarrow 0+.
$$

(2.73) may not be valid near $x=1$. Then we have to find an upper limit for the region in which (2.73) is valid. To do this, we let $Q(x) \sim a x+b x^{2}$. Hence

$$
\int_{0}^{x} \sqrt{a t+b t^{2}} d t \sim \int_{0}^{x} \sqrt{a t}\left(1+\frac{b t}{2 a}\right) d t=\frac{2}{3} a^{\frac{1}{2}} x^{\frac{3}{2}}+\frac{b}{5 \sqrt{a}} x^{\frac{5}{2}}, \quad x \rightarrow 0+.
$$

In order that (2.73) be satisfied, we must have

$$
e^{\frac{-2}{3 \epsilon} a^{\frac{1}{2}} x^{\frac{3}{2}}-\frac{1}{5 \epsilon} b a^{\frac{-1}{2}} x^{\frac{5}{2}}} \sim e^{\frac{-2}{3 \epsilon} a^{\frac{1}{2}} x^{\frac{3}{2}}}
$$

or

$$
\frac{1}{\epsilon} x^{5 / 2} \ll 1
$$

which implies $x \ll \epsilon^{\frac{2}{5}}$, as $\epsilon \rightarrow 0$.

Thus the overlap region in which (2.73) is valid is $\epsilon^{\frac{2}{3}} \ll x \ll \epsilon^{\frac{2}{5}}$.

On the other hand, in the intermediate region, where $t \rightarrow \infty$, we may use the following approximations for the Airy functions (see the Appendix: (6.22)):

$$
\begin{array}{ll}
\operatorname{Ai}(t) \sim \frac{1}{2 \sqrt{\pi}} t^{\frac{-1}{4}} e^{\frac{-2}{3} t^{\frac{3}{2}}}, & t \rightarrow+\infty, \\
\operatorname{Bi}(t) \sim \frac{1}{\sqrt{\pi}} t^{\frac{-1}{4}} e^{\frac{2}{3} t^{\frac{3}{2}}}, & t \rightarrow+\infty .
\end{array}
$$


By substituting these formula in (2.72), we get

$$
y_{\mathrm{II}} \sim \frac{1}{\sqrt{\pi}} a^{\frac{-1}{12}} \epsilon^{\frac{1}{6}} x^{\frac{-1}{4}}\left(\frac{1}{2} D e^{\frac{-2}{3 \epsilon} a^{\frac{1}{2}} x^{\frac{3}{2}}}+E e^{\frac{2}{3 \epsilon} a^{\frac{1}{2}} x^{\frac{3}{2}}}\right) .
$$

By using the change of variable in (2.70), the lower bound of $(2.74)$ is $x \gg \epsilon^{\frac{2}{3}}$. Then the valid region for $(2.74)$ is $\epsilon^{\frac{2}{3}} \ll x \ll 1$. Therefore $y_{\text {I }}$ and $y_{\text {II }}$ have an overlap region specified by

$$
\epsilon^{\frac{2}{3}} \ll x \ll \epsilon^{\frac{2}{5}}
$$

(See the remark 2.5 for more details.)

Now we equate (2.73) and (2.74) in the overlap region of $y_{\mathrm{I}}$ and $y_{\mathrm{II}}$. Hence

$$
\begin{aligned}
& D=2 C \sqrt{\pi}(a \epsilon)^{\frac{-1}{6}}, \\
& E=0 .
\end{aligned}
$$

In summary:

$$
y_{\mathrm{II}}(x) \sim 2 C \sqrt{\pi}(a \epsilon)^{\frac{-1}{6}} \operatorname{Ai}\left(a^{\frac{1}{3}} \epsilon^{\frac{-2}{3}} x\right), \quad x \rightarrow 0 .
$$

Now to find an approximation to $y_{\mathrm{III}}$, we use the CASE I. By the assumptions we made at the beginning of the CASE II, we know that $-Q(x)>0$ when $x<0$. Thus, for $\epsilon \ll 1$ and $x \rightarrow-\infty$, we obtain

$$
y_{\mathrm{III}}(x) \sim F(-Q(x))^{\frac{-1}{4}} e^{\frac{i}{\epsilon} \int_{x}^{0} \sqrt{-Q(t)} d t}+G(-Q(x))^{\frac{-1}{4}} e^{\frac{-i}{\epsilon} \int_{x}^{0} \sqrt{-Q(t)} d t}
$$

for some integral constants $F$ and $G$.

$$
\begin{aligned}
y_{\mathrm{III}}(x) \sim(-Q(x))^{\frac{-1}{4}}\left((F+G) \cos \left(\frac{1}{\epsilon} \int_{x}^{0} \sqrt{-Q(t)} d t\right)+\right. \\
\left.i(F-G) \sin \left(\frac{1}{\epsilon} \int_{x}^{0} \sqrt{-Q(t)} d t\right)\right),
\end{aligned}
$$

or equivalently,

$$
y_{\mathrm{III}}(x) \sim R(-Q(x))^{\frac{-1}{4}} \sin \left(\frac{1}{\epsilon} \int_{x}^{0} \sqrt{-Q(t)} d t+\theta\right),
$$


for some constants $R$ and $\theta$.

On the other side, for $x \rightarrow 0^{-}, \epsilon \rightarrow 0^{+}$and $t \rightarrow-\infty$, we have $\int_{x}^{0} \sqrt{-Q(t)} d t \sim$ $\frac{-2}{3} a^{\frac{1}{2}}(-x)^{\frac{3}{2}}$. Thus

$$
y_{\mathrm{III}}(x) \sim R a^{\frac{-1}{4}}(-x)^{\frac{1}{4}} \sin \left(\frac{-2}{3 \epsilon} a^{\frac{1}{2}}(-x)^{\frac{3}{2}}+\theta\right) .
$$

In addition, $S_{2}(x) \sim \pm \frac{5}{48} a^{\frac{-1}{2}}(-x)^{\frac{-3}{2}}$. Hence, (similar to (2.69)) the valid region of $y_{\mathrm{III}}$ is $(-x) \gg \epsilon^{\frac{2}{3}}$.

Referring to the remark (6.6), an approximation to the general solution of the Airy equation as $t \rightarrow-\infty$ is

$$
y_{\mathrm{II}}(t) \sim \pi^{-\frac{1}{2}}(-t)^{-\frac{1}{4}}\left(D \sin \left(\frac{2}{3}(-t)^{\frac{3}{2}}+\frac{1}{4} \pi\right)+E \cos \left(\frac{2}{3}(-t)^{\frac{3}{2}}+\frac{1}{4} \pi\right)\right),
$$

$t \rightarrow-\infty$.

or

$$
\begin{array}{r}
y_{\mathrm{II}}(x) \sim \pi^{-\frac{1}{2}} a^{\frac{-1}{12}} \epsilon^{\frac{1}{6}}(-x)^{-\frac{1}{4}}\left(D \sin \left(\frac{2}{3 \epsilon} a^{\frac{1}{2}}(-x)^{\frac{3}{2}}+\frac{1}{4} \pi\right)+E \cos \left(\frac{2}{3 \epsilon} a^{\frac{1}{2}}(-x)^{\frac{3}{2}}+\frac{1}{4} \pi\right)\right), \\
x \rightarrow-\infty
\end{array}
$$

By comparing (2.77) and (2.76) in the overlap region which is $\epsilon^{\frac{2}{3}} \ll(-x) \ll \epsilon^{\frac{2}{5}}$ and choosing $R=2 C, \theta=\frac{\pi}{4}, D=2 C \sqrt{\pi}(a \epsilon)^{\frac{-1}{6}}$ and $E=0, y_{\text {III }}$ and $y_{\text {II }}$ are successfully matched in the mentioned intermediate region.

In summary, we have found an approximation to $y(x)$ in 3 regions:

$$
\begin{aligned}
& y_{\mathrm{I}}(x)=C(Q(x))^{\frac{-1}{4}} e^{\frac{-1}{\epsilon} \int_{0}^{x} \sqrt{Q(t)} d t}, x \gg \epsilon^{\frac{2}{3}}, \\
& y_{\mathrm{II}}(x)=2 C \sqrt{\pi}(a \epsilon)^{\frac{-1}{6}} \operatorname{Ai}\left(\epsilon^{\frac{-2}{3}} a^{\frac{1}{3}} x\right),|x| \ll 1, \\
& y_{\mathrm{III}}(x)=2 C(-Q(x))^{\frac{-1}{4}} \sin \left(\frac{1}{\epsilon} \int_{x}^{0} \sqrt{-Q(t)} d t+\frac{\pi}{4}\right),(-x) \gg \epsilon^{\frac{2}{3}} .
\end{aligned}
$$

Having an additional boundary or initial condition can determine a specific solution to (2.62). 
Remark 2.5. We must check the intermediate region does not vanish as $\epsilon \rightarrow 0$. Indeed, the size of the overlap region, in terms of $t$, must tend to infinity as $\epsilon \rightarrow 0$. By substituting $t$ into (2.75), we find

$$
\epsilon^{\frac{2}{3}} \ll \epsilon^{\frac{2}{3}} t \ll \epsilon^{\frac{2}{5}}
$$

which implies

$$
1 \ll t \ll \epsilon^{\frac{-4}{15}}
$$

Then $t$ is free to become large as $\epsilon \rightarrow 0+$.

\subsubsection{The RG Method Applied to the Time-Independent Schrödinger Equation}

Now we are going to apply the RG method to approximate solutions of the Schrödinger equation in both cases, see [12].

CASE I). There is no turning point. Recall that the Schrödinger equation is

$$
\epsilon^{2} u^{\prime \prime}-Q(x) u=0, \quad \epsilon \ll 1,^{2}
$$

where $Q(x)$ is a smooth and slowly varying function. In equation (2.78), the highest derivative term is multiplied by a small parameter. As we mentioned in the boundary layer section, we need to use a change of variable which leads a new equation without a boundary layer structure. To do this, let $t=f(x)$. We find $t$ implicitly considering that the new equation converts to

$$
\frac{d^{2} u}{d t^{2}}-u=\epsilon(g(x(t)))
$$

for some function $g$.

\footnotetext{
${ }^{2}$ Imposing the change of variable $X=\frac{x}{\epsilon}$, the Schrödinger equation becomes dimensionless: $u^{\prime \prime}(X)-Q(\epsilon X) u(X)=0$
} 
By taking the derivative from (2.78) with respect to $t$ and by using the Chain Rule, we find

$$
\epsilon^{2} \frac{f^{\prime \prime}(x)}{Q(x)} \frac{d u}{d t}+\epsilon^{2} \frac{f^{\prime 2}(x)}{Q(x)} \frac{d^{2} u}{d t^{2}}-u=0
$$

To get(2.79), we set: $\epsilon^{2} f^{\prime 2}(x)=Q(x)$ and hence $f^{\prime}(x)=\frac{\sqrt{Q(x)}}{\epsilon}$ and $f^{\prime \prime}(x)=$ $\frac{1}{2 \epsilon} Q^{\prime} Q^{\frac{-1}{2}}$. Therefore

$$
t=f(x)=\frac{1}{\epsilon} \int_{0}^{x} \sqrt{Q(v)} d v
$$

Now by inserting the values of $f^{\prime}(x)$ and $f^{\prime \prime}(x)$ into (2.80), we obtain

$$
\frac{d^{2} u}{d t^{2}}-u=2 \epsilon S(x) \frac{d u}{d t}
$$

where $S(x(t))=-\frac{1}{4} Q^{-\frac{3}{2}}(x) Q^{\prime}(x)$.

Now we apply the RG method to (2.81). Let

$$
u(t)=u_{0}(t)+\epsilon u_{1}(t)+\mathcal{O}\left(\epsilon^{2}\right) .
$$

Substituting (2.82) into (2.81) implies

$$
\begin{aligned}
& \frac{d^{2} u_{0}}{d t^{2}}-u_{0}=0 \\
& \frac{d^{2} u_{1}}{d t^{2}}-u_{1}=2 S(x(t)) \frac{d u_{0}}{d t} .
\end{aligned}
$$

The solutions to (2.83) can be written

$$
u_{0}(t)=A_{0} e^{t}+B_{0} e^{-t},
$$

and

$$
\begin{array}{r}
u_{1}(t)=A_{0} A_{1} e^{t}+B_{0} B_{1} e^{-t}+A_{0} e^{t}\left(\int_{t_{0}}^{t} S(x(r)) d r-e^{-2 t} \int_{t_{0}}^{t} S(x(r)) e^{2 r} d r\right)+ \\
B_{0} e^{-t}\left(\int_{t_{0}}^{t} S(x(r)) d r-e^{2 t} \int_{t_{0}}^{t} S(x(r)) e^{-2 r} d r\right) .
\end{array}
$$


By substituting $u_{0}$ and $u_{1}$, the first order solution reads

$$
\begin{array}{r}
u(t)=A_{0} e^{t}+B_{0} e^{-t}+A_{0} \epsilon e^{t}\left(A_{1}+\int_{t_{0}}^{t} S(x(r)) d r-e^{-2 t} \int_{t_{0}}^{t} S(x(r)) e^{2 r} d r\right)+ \\
B_{0} \epsilon e^{-t}\left(B_{1}+\int_{t_{0}}^{t} S(x(r)) d r-e^{2 t} \int_{t_{0}}^{t} S(x(r)) e^{-2 r} d r\right)+\mathcal{O}\left(\epsilon^{2}\right) .
\end{array}
$$

By using integration by parts infinitely many times, we find

$$
\begin{gathered}
e^{-2 t} \int_{t_{0}}^{t} S(x(r)) e^{2 r} d r=e^{-2 t} C_{0}+\sum_{n=0}^{\infty} \frac{(-1)^{n}}{2^{n+1}}(S(x(t)))^{(n)}, \\
e^{2 t} \int_{t_{0}}^{t} S(x(r)) e^{-2 r} d r=e^{2 t} C_{1}-\sum_{n=0}^{\infty} \frac{1}{2^{n+1}}(S(x(t)))^{(n)},
\end{gathered}
$$

for some constants $C_{0}$ and $C_{1}$ and $S$ is a smoothly and slowly varying function. If the derivatives are bounded such that $\left\|S^{(n)}\right\|_{\infty}<c^{n}, c<2$, then the series are convergent.

For $t-t_{0} \rightarrow \infty$, terms $F(t)=-e^{-2 t} \int_{t_{0}}^{t} S(x(r)) e^{2 r} d r$ and $G(t)=-e^{2 t} \int_{t_{0}}^{t} S(x(r)) e^{-2 r} d r$ are smaller than $\int_{t_{0}}^{t} S(x(r)) d r$, which causes secular terms.

From now on, for ease of notation, we write

$$
\begin{aligned}
u(t)=A_{0} e^{t}+B_{0} e^{-t}+A_{0} \epsilon e^{t}\left(A_{1}+\right. & \left.\int_{t_{0}}^{t} S(x(r)) d r+F(t)\right)+ \\
& B_{0} \epsilon e^{-t}\left(B_{1}+\int_{t_{0}}^{t} S(x(r)) d r+G(t)\right)+\mathcal{O}\left(\epsilon^{2}\right) .
\end{aligned}
$$

To absorb secular terms, we introduce the new free parameter

$$
\begin{gathered}
u(t)=A_{0} e^{t}+B_{0} e^{-t}+A_{0} \epsilon e^{t}\left(A_{1}+\int_{t_{0}}^{\sigma} S(x(r)) d r+\right. \\
\left.\int_{\sigma}^{t} S(x(r)) d r+F(t)\right)+B_{0} \epsilon e^{-t}\left(B_{1}+\int_{t_{0}}^{\sigma} S(x(r)) d r+\right. \\
\left.\int_{\sigma}^{t} S(x(r)) d r+G(t)\right)+\mathcal{O}\left(\epsilon^{2}\right) .
\end{gathered}
$$

Now we introduce multiplicative renormalization constants

$$
\begin{aligned}
& A_{0}=A(\sigma)+a \epsilon A(\sigma)+\mathcal{O}\left(\epsilon^{2}\right), \\
& B_{0}=B(\sigma)+b \epsilon B(\sigma)+\mathcal{O}\left(\epsilon^{2}\right) .
\end{aligned}
$$


Similar to previous examples, we can substitute (2.85) into (2.84) and choose values for $a$ and $b$ as

$$
\begin{aligned}
& a=-\left(A_{1}+\int_{t_{0}}^{\sigma} S(x(r)) d r\right), \\
& b=-\left(B_{1}+\int_{t_{0}}^{\sigma} S(x(r)) d r\right) .
\end{aligned}
$$

to obtain

$$
\begin{aligned}
& u(t)=e^{t}\left(A+A \epsilon \int_{\sigma}^{t} S(x(r)) d r+A \epsilon F(t)\right)+e^{-t}(B+ \\
&\left.B \epsilon \int_{\sigma}^{t} S(x(r)) d r+B \epsilon G(t)\right)+\mathcal{O}\left(\epsilon^{2}\right) .
\end{aligned}
$$

Applying the RG condition, $\frac{\partial u}{\partial \sigma}=\mathcal{O}\left(\epsilon^{2}\right)$, gives

$$
\begin{aligned}
e^{t}\left(\frac{d A}{d \sigma}+\epsilon \frac{d A}{d \sigma} \int_{\sigma}^{t} S(x(r))\right. & \left.d r-\epsilon A S(x(\sigma))+\frac{d A}{d \sigma} \epsilon F(t)\right)+e^{-t}\left(\frac{d B}{d \sigma}+\right. \\
\left.\epsilon \frac{d B}{d \sigma} \int_{\sigma}^{t} S(x(r)) d r-\epsilon B S(x(\sigma))+\frac{d B}{d \sigma} \epsilon G(t)\right) & =\mathcal{O}\left(\epsilon^{2}\right) .
\end{aligned}
$$

Since $e^{t}$ and $e^{-t}$ are linearly independent functions, we come to realize that

$$
\begin{aligned}
& \frac{d A}{d \sigma}-\epsilon A S(x(\sigma))=\mathcal{O}\left(\epsilon^{2}\right), \\
& \frac{d B}{d \sigma}-\epsilon B S(x(\sigma))=\mathcal{O}\left(\epsilon^{2}\right) .
\end{aligned}
$$

$A$ and $B$ are solutions to an equivalent ODE. As a result, we have only to find $A$. By setting $\sigma=t$, and using the definition of $t$ and $S$ we obtain

$$
\frac{\epsilon}{Q^{\frac{1}{2}}} \frac{d A}{d x}=-\frac{\epsilon}{4} Q^{\prime} Q^{-\frac{3}{2}} A+\mathcal{O}\left(\epsilon^{2}\right) .
$$

Thus

$$
\frac{d A}{d x}=-\frac{1}{4} Q^{\prime} Q^{-1} A+\mathcal{O}\left(\epsilon^{2}\right)
$$

(2.86) is a linear first order differential equation. Hence 


$$
B(x)=A(x)=e^{-\frac{1}{4} \int Q^{\prime}(x) Q^{-1}(x) d x}+\mathcal{O}\left(x \epsilon^{2}\right)=Q^{-\frac{1}{4}}(x)+\mathcal{O}\left(x \epsilon^{2}\right) .
$$

Therefore

$$
u(x)=C_{1} Q^{-\frac{1}{4}}(x) e^{t}+C_{2} Q^{-\frac{1}{4}}(x) e^{-t}+\mathcal{O}\left(x \epsilon^{2}\right)
$$

or

$$
u(x)=Q^{-\frac{1}{4}}(x)\left(C_{1} e^{\frac{1}{\epsilon} \int^{x} \sqrt{Q(r)} d r}+C_{2} e^{-\frac{1}{\epsilon} \int^{x} \sqrt{Q(r)} d r}\right)+\mathcal{O}\left(x \epsilon^{2}\right) .
$$

This shows that the results of the WKB theory and RG method are identical in the case of no turning points (see (2.65).)

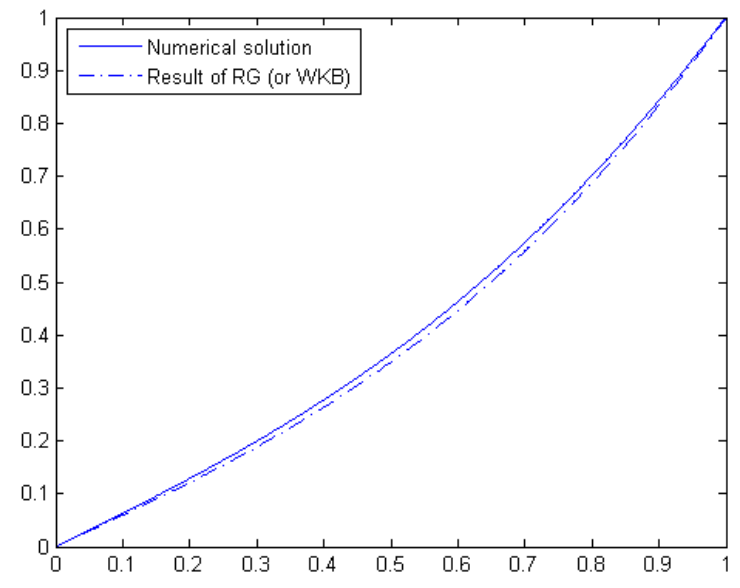

Figure 2.8: A comparison between plots of the numerical solution to the Schrödinger equation using the Shooting Method and RG outcome (which is equivalent to WKB's outcome), when $Q(x)=\frac{1}{\cosh ^{2}(x)}$ and $\epsilon=.5$.

CASE II. There exists one turning point. Recall the Schrödinger equation having a boundary condition at $\infty$ :

$$
\epsilon^{2} \frac{d^{2} u}{d x^{2}}=Q(x) u, \quad u(\infty)=0
$$


such that $Q$ is an analytic and has only one root at $x=0$. Then we can write $Q(x)=x^{\alpha} \phi(x)$, for some $\alpha$ and nowhere zero function $\phi$. Here we use a change of variable such that only $x^{\alpha}$ remains in the right hand side of (2.87); by this change of variable, the solutions will have the Airy functions type behavior. Let $t=f(x)$ be such that $t \sim x$ as $x \rightarrow 0$. Then we have

$$
\epsilon^{2}\left(f^{\prime \prime} \frac{d u}{d t}+\left(f^{\prime}\right)^{2} \frac{d^{2} u}{d t^{2}}\right)=Q(x) u(t)
$$

where' stands for $\frac{d}{d x}$.

To eliminate this singularity, we multiply both sides by the factor $t^{\alpha}$ to obtain

$$
\epsilon^{2} \frac{f^{\prime \prime} t^{\alpha}}{Q} \frac{d u}{d t}+\epsilon^{2} \frac{\left(f^{\prime}\right)^{2} t^{\alpha}}{Q} \frac{d^{2} u}{d t^{2}}=t^{\alpha} u(t)
$$

Since $\frac{t^{\alpha}}{x^{\alpha}} \sim 1$ as $x \rightarrow 0$, then the coefficients are no longer unbounded. We find $f$ by requiring that the coefficient of $\frac{d^{2} u}{d t^{2}}$ be 1 . Thus

$$
t(x)=\left(\frac{\alpha+2}{2 \epsilon} \int_{0}^{x} \sqrt{Q(r)} d r\right)^{\frac{2}{\alpha+2}} .
$$

In addition

$$
\frac{\epsilon^{2} f^{\prime \prime} t^{\alpha}}{Q}=\epsilon \frac{t^{\alpha}}{Q} \frac{d}{d x} \sqrt{\frac{Q}{t^{\alpha}}}=-\epsilon \frac{d}{d x} \sqrt{\frac{t^{\alpha}}{Q}} .
$$

If we name $S(t(x))=\frac{d}{d x} \sqrt{\frac{t^{\alpha}}{Q}}$, we get

$$
\frac{d^{2} u}{d t^{2}}=t^{\alpha} u(t)+\epsilon S(t(x)) \frac{d u}{d t} .
$$

In the last subsection we assumed that the solution has a simple root. Thus we let $\alpha=1$. Now we try a naive perturbation for the solution to $(2.88)$

$$
u(t)=u_{0}(t)+\epsilon u_{1}(t)+\mathcal{O}\left(\epsilon^{2}\right) .
$$

Inserting (2.89) into (2.88) gives

$$
\begin{aligned}
& u_{0}^{\prime \prime}-t u_{0}=0, \\
& u_{1}^{\prime \prime}-t u_{1}=S(t) u_{0}^{\prime},
\end{aligned}
$$


where $^{\prime}=\frac{d}{d t}$.

The general solutions to $(2.90)$ can be written as

$$
\begin{aligned}
& u_{0}=C_{0} \operatorname{Ai}(t)+D_{0} \operatorname{Bi}(t), \\
& u_{1}=C_{0} C_{1} \operatorname{Ai}(t)+D_{1} \operatorname{Bi}(t)+u_{p},
\end{aligned}
$$

where $u_{p}$ is a particular solution to the nonhomogeneous equation (2.90b). In addition, since $\operatorname{Bi}(t)$ is an unbounded function for large values of $t$, and since $u(\infty)=0$, we must have $D_{0}=D_{1}=0$.

Using the method of variation of parameters, we have $u_{p}=V_{1} \operatorname{Ai}(t)+V_{2} \operatorname{Bi}(t)$ where

$$
\begin{aligned}
V_{1}^{\prime} & =\frac{-\operatorname{Bi}(t) S(t) u_{0}^{\prime}}{w}, \\
V_{2}^{\prime} & =\frac{\operatorname{Ai}(t) S(t) u_{0}^{\prime}}{w},
\end{aligned}
$$

and $w$ is the Wronskian of the homogeneous solutions. Since

$$
w=\mathrm{AiBi}^{\prime}-\mathrm{BiAi}^{\prime}=\frac{1}{\pi},
$$

(see the remark (6.5)), hence

$$
\begin{aligned}
& V_{1}(t)=-\pi C_{0} \int_{t_{0}}^{t} \operatorname{Bi}(r) S(r) \mathrm{Ai}^{\prime} d r \\
& V_{2}(t)=\pi C_{0} \int_{t_{0}}^{t} \operatorname{Ai}(r) S(r) \mathrm{Ai}^{\prime} d r .
\end{aligned}
$$

Therefore

$$
\begin{aligned}
u(t)=C_{0} \operatorname{Ai}(t)+\pi C_{0} \epsilon\left(C_{1} \operatorname{Ai}(t)-\operatorname{Ai}(t) \int_{t_{0}}^{t} \operatorname{Bi}(r) S(r) \operatorname{Ai}^{\prime}(r) d r+\right. \\
\\
\left.\quad \operatorname{Bi}(t) \int_{t_{0}}^{t} \operatorname{Ai}(r) S(r) \operatorname{Ai}^{\prime}(r) d r\right)+\mathcal{O}\left(\epsilon^{2}\right) .
\end{aligned}
$$

According to the definition, $S(t)$ is an analytic function. Hence we have $S(t)=$ $\sum_{n=0}^{\infty} c_{n} t^{n}$. The term $\operatorname{Bi} \int_{t_{0}}^{t} \operatorname{Ai}(r) S(r) \mathrm{Ai}^{\prime} d r$ remains bounded and $\mathrm{Ai} \int_{t_{0}}^{t} \operatorname{Bi}(r) S(r) \mathrm{Ai}^{\prime} d r$ causes secular term(s) as $t \rightarrow \infty$. (See the remark 2.6) 
Thus $\mathrm{Bi} \int_{t_{0}}^{t} \operatorname{Ai}(r) S(r) \mathrm{Ai}^{\prime} d r$ does not result in secular term(s) while $\operatorname{Ai}(t) \int_{t_{0}}^{t} \operatorname{Bi}(r) S(r) \mathrm{Ai}^{\prime}(r) d r$ causes secular term(s) and then must be renormalized. Rewrite (2.92) as

$$
u(t)=C_{0} \operatorname{Ai}(t)+\pi C_{0} \epsilon\left(C_{1} \operatorname{Ai}(t)-\operatorname{Ai}(t) \int_{t_{0}}^{t} \operatorname{Bi}(r) S(r) \mathrm{Ai}^{\prime}(r) d r+F(t)\right)+\mathcal{O}\left(\epsilon^{2}\right),
$$

where $F(t)=\operatorname{Bi}(t) \int_{t_{0}}^{t} \operatorname{Ai}(r) S(r) \mathrm{Ai}^{\prime} d r$ is bounded in $t$.

We introduce the new parameter $\sigma$ and split the integrals

$$
\begin{aligned}
u(t)=C_{0} \operatorname{Ai}(t)+\pi C_{0} \epsilon\left(C_{1} \operatorname{Ai}(t)-\operatorname{Ai}(t) \int_{t_{0}}^{\sigma} \operatorname{Bi}(r) S(r) \mathrm{Ai}^{\prime}(r) d r-\right. \\
\left.\operatorname{Ai}(t) \int_{\sigma}^{t} \operatorname{Bi}(r) S(r) \mathrm{Ai}^{\prime}(r) d r+F(t)\right)+\mathcal{O}\left(\epsilon^{2}\right) .
\end{aligned}
$$

Let

$$
C_{0}=C(\sigma)+a \epsilon C(\sigma)+\mathcal{O}\left(\epsilon^{2}\right)
$$

If we choose $a=\pi\left(-C_{1}+\int_{t_{0}}^{\sigma} \operatorname{Bi}(r) S(r) \mathrm{Ai}^{\prime}(r) d r\right),(2.91)$ reduces to

$$
u(t)=C \operatorname{Ai}(t)-C \pi \epsilon\left(\operatorname{Ai}(t) \int_{\sigma}^{t} \operatorname{Bi}(r) S(r) \operatorname{Ai}^{\prime}(r) d r+F(t)\right)+\mathcal{O}\left(\epsilon^{2}\right)
$$

By applying the RG condition and observing that $F(t)$ does not contain $\sigma$, we obtain

$$
\frac{d C(\sigma)}{d \sigma}+\pi \epsilon S(\sigma) \operatorname{Ai}^{\prime}(\sigma) \operatorname{Bi}(\sigma) C(\sigma)=\mathcal{O}\left(\epsilon^{2}\right)
$$

Hence

$$
C(t)=c e^{-\pi \epsilon \int_{0}^{t} S(r) \operatorname{Ai}^{\prime}(r) \operatorname{Bi}(r) d r}+\mathcal{O}\left(t \epsilon^{2}\right)
$$

To substitute the value of $S$ in the above formula, we would write $S$ as a derivative of some function with respect to $t$ rather than $x$. If $v=\left(\frac{t}{Q}\right)^{1 / 2}$, then we have $S(x(t))=\frac{d}{d x}\left(\frac{t}{Q}\right)^{\frac{1}{2}}=\frac{d v}{d x}=\frac{d v}{d t} \frac{d t}{d x}=\frac{1}{\epsilon} \frac{1}{v} \frac{d v}{d t}=\frac{1}{\epsilon} \frac{d}{d t} \ln v$.

Thus

$$
C(t)=C(0) e^{-\pi \int_{0}^{t} \operatorname{Ai}^{\prime}(r) \operatorname{Bi}(r) \frac{d}{d r} \ln \left(\frac{r}{Q}\right)^{1 / 2} d r}+\mathcal{O}\left(t \epsilon^{2}\right)
$$


Therefore the zeroth order solution to $(2.87)$ is

$$
u(x)=C(0) \operatorname{Ai}(t) e^{-\pi \int_{0}^{t} \operatorname{Ai}(r) \operatorname{Bi}(r) \frac{d}{d r} \ln \left(\frac{r}{Q}\right)^{1 / 2} d r}+\mathcal{O}\left(t \epsilon^{2}\right),
$$

where $t=\left(\frac{3}{2 \epsilon} \int_{0}^{x} \sqrt{Q(r)} d r\right)^{\frac{2}{3}}$ and $C(0)$ can be determined by an additional boundary condition. See the figures (2.9) and (2.10) to observe that the outcomes of WKB and $\mathrm{RG}$ are close to each other for all times.

Remark 2.6. We substitute the Taylor expansion of $S$ into the integrals. Thus we obtain

$$
\operatorname{Bi}(t) \int_{t_{0}}^{t} \sum_{n=0}^{\infty} c_{n} r^{n} \operatorname{Ai}(r) \operatorname{Ai}^{\prime}(r) d r=\operatorname{Bi}(t) \sum_{n=0}^{\infty} c_{n} \int_{t_{0}}^{t} r^{n} \operatorname{Ai}(r) \operatorname{Ai}^{\prime}(r) d r
$$

Since $\operatorname{Ai}(r) \operatorname{Ai}^{\prime}(r)$ decays exponentially (see the Appendix (6.22)), for every $n \geq 0$, we have

$$
\operatorname{Bi}(t) \int_{t_{0}}^{t} r^{n} \operatorname{Ai}(r) \operatorname{Ai}^{\prime}(r) d r \sim \operatorname{Bi}(t) \int_{t_{0}}^{t} \operatorname{Ai}(r) \operatorname{Ai}^{\prime}(r) d r=\frac{1}{2} \operatorname{Bi}(t)(\operatorname{Ai}(t))^{2} .
$$

For the other integral, since $\operatorname{Bi}(r) \mathrm{Ai}^{\prime}(r)=\frac{-1}{4} r^{-\frac{3}{2}}-1$ (see (6.22)), it turns out

$$
\begin{aligned}
\operatorname{Ai}(t) \int_{t_{0}}^{t} r^{n} \operatorname{Bi}(r) \operatorname{Ai}^{\prime}(r) d r \sim \operatorname{Ai}(t) \int_{t_{0}}^{t}\left(\frac{-1}{4} r^{-\frac{3}{2}}-1\right) r^{n} d r= \\
\quad\left(\frac{-1}{2 n-2} t^{n-\frac{1}{2}}-\frac{1}{n+1} t^{n+1}\right) \operatorname{Ai}(t),
\end{aligned}
$$

which is a secular term. Thus

$$
\begin{aligned}
u(t) \sim \operatorname{Ai}(t)\left(C_{0}+\pi C_{0} \epsilon\left(C_{1}-\sum_{n=0}^{\infty} \frac{c_{n}}{2} \operatorname{Bi}(t)(\operatorname{Ai}(t))^{2}+\right.\right. & \\
& \left.\left.\sum_{n=0}^{\infty} \frac{c_{n}}{n+1} t^{n+1}\right)\right)+\mathcal{O}\left(\epsilon^{2}\right) .
\end{aligned}
$$

\subsection{Nonlinear Carrier Equation}

In this section we will show how RG can be applied to a nonlinear differential equation to which WKB cannot be applied. Consider the Carrier equation 


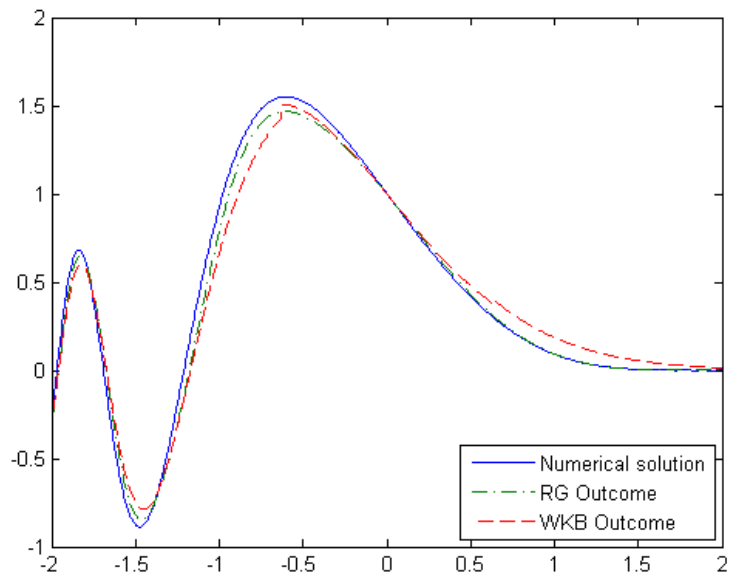

Figure 2.9: A comparison of the RG method with the WKB approximation, applied to the Schrödinger equation when the potential $Q(x)=\sinh (x) \cosh ^{2}(x)$, $y(+\infty)=0, y(0)=1$ and $\epsilon=.2$.

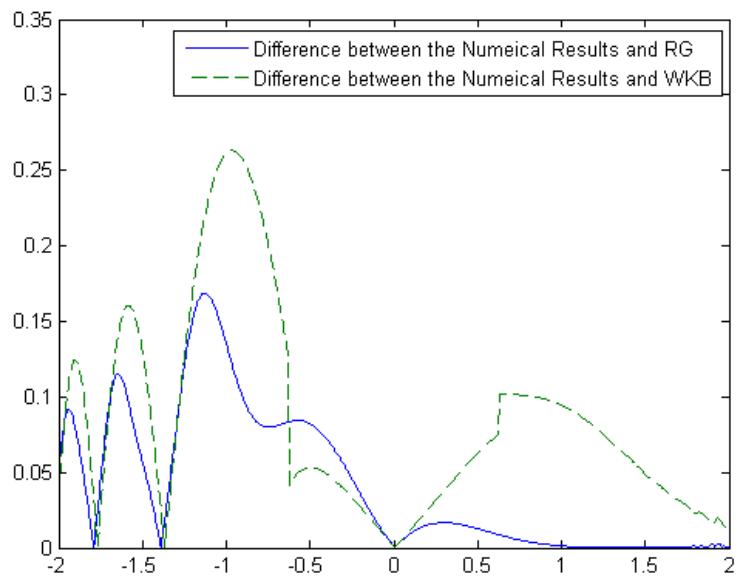

Figure 2.10: The difference between the RG method's and WKB theory's outcomes. 


$$
(x+\epsilon y) y^{\prime}+y=1, \quad y(1)=2, \quad 0 \leq x \leq 1
$$

The naive perturbative expansion will fail due to a secular term. Then we need a perturbation method which renormalizes the secularity. One may suggest to apply the multiple scale analysis! To apply the multiple scale analysis, we need to define a new independent variable (time scale) $X=\epsilon x$ and then to define an expansion with respect to $x$ as well as $X$. The other option is to apply the RG method to this equation.

However, in the first subsection, we solve the equation by elementary techniques. In the second subsection we will apply the RG method which reproduces the exact solution of the nonlinear Carrier equation.

\subsubsection{The Exact Solution}

One way to solve the Carrier differential equation (2.93) is to use the equality: $(x+$ $\epsilon y)^{\prime}=1+\epsilon y^{\prime}$. Hence $y^{\prime}=\frac{1}{\epsilon}\left((x+\epsilon y)^{\prime}-1\right)$. Now we insert this into (2.94)

$$
\begin{aligned}
(x+\epsilon y) y^{\prime}+y=\frac{1}{\epsilon}\left((x+\epsilon y)^{\prime}-1\right)(x+\epsilon y)+y & =1, \\
& \Rightarrow(x+\epsilon y)^{\prime}(x+\epsilon y)=\epsilon+x .
\end{aligned}
$$

Integrating with respect to $x$ gives

$$
y=\frac{1}{\epsilon}\left(\sqrt{2 \epsilon x+x^{2}+2 c}-x\right) .
$$

Using the boundary condition, we find the specific solution as follow

$$
y=-\frac{x}{\epsilon}+\sqrt{\frac{x^{2}}{\epsilon^{2}}+\frac{2(x+1)}{\epsilon}+4} .
$$




\subsubsection{The RG method Applied to the Carrier Equation}

In this subsection, we are going to apply the RG method to deduce (2.95), see [12].

To begin with, we make the two change of variables: $X=\eta x$ and $Y=\delta y$. We insert them into (2.93) and try to simplify the equation using the method of dominant balance

$$
\left(\frac{X}{\eta}+\epsilon \frac{Y}{\delta}\right) \frac{\eta}{\delta} Y^{\prime}+\frac{Y}{\delta}=1 \Rightarrow\left(X+\frac{\eta \epsilon}{\delta} Y\right) Y^{\prime}+Y=\delta .
$$

By having $\frac{\eta \epsilon}{\delta} \ll 1$ we loose the nonlinear term's efficacy and by having $\frac{\eta \epsilon}{\delta} \gg 1$ we find the outer solution. Then the only consistent choice is $\frac{\eta \epsilon}{\delta} \sim 1$. We can pick up $\delta=\epsilon$ and $\eta=1$; hence

$$
(x+Y) Y^{\prime}+Y=\epsilon, \quad Y(1)=2 \epsilon .
$$

Now we write the solution to (2.96) as a naive perturbative expansion

$$
Y(x)=Y_{0}(x)+\epsilon Y_{1}(x)+\mathcal{O}\left(\epsilon^{2}\right) .
$$

Thus we obtain

$$
\begin{aligned}
\left(x+Y_{0}\right) Y_{0}^{\prime}+Y_{0} & =0 \\
\left(Y_{0}+x\right) Y_{1}^{\prime}+\left(Y_{0}^{\prime}+1\right) Y_{1} & =1 .
\end{aligned}
$$

The solutions to (2.97) are

$$
\begin{aligned}
& Y_{0}=-x+\sqrt{A_{0}+x^{2}}, \\
& Y_{1}=\frac{A_{0} A_{1}}{\sqrt{A_{0}+x^{2}}}+\frac{x-x_{0}}{\sqrt{A_{0}+x^{2}}},
\end{aligned}
$$

where $x_{0}$ is an arbitrary initial point and $A_{0}=A_{0}\left(x_{0}\right)$ and $A_{1}=A_{1}\left(x_{0}\right)$ are the integral constants depending on the initial point. 
Hence

$$
Y(x)=-x+\sqrt{A_{0}+x^{2}}+\epsilon\left(\frac{A_{0} A_{1}}{\sqrt{A_{0}+x^{2}}}+\frac{x-x_{0}}{\sqrt{A_{0}+x^{2}}}\right)+\mathcal{O}\left(\epsilon^{2}\right) .
$$

Since the range of integration is bounded, it seems that the term $\frac{x-x_{0}}{\sqrt{A_{0}+x^{2}}}$ is not a secular term. But by doing some analysis we come to realize that there is a secularity near $x=1$ : Since $Y(1)=2 \epsilon$, thus $Y_{0}(1)=\mathcal{O}\left(\epsilon^{2}\right)$ and $Y_{1}(1)=2+\mathcal{O}(\epsilon)$ which imply that $Y_{1}$ is much greater than $Y_{0}$ near $x=1$.

Now, to absorb the secularity, we introduce the new parameter

$$
Y(x)=-x+\sqrt{A_{0}+x^{2}}+\epsilon\left(\frac{A_{0} A_{1}}{\sqrt{A_{0}+x^{2}}}+\frac{\sigma-x_{0}}{\sqrt{A_{0}+x^{2}}}+\frac{x-\sigma}{\sqrt{A_{0}+x^{2}}}\right)+\mathcal{O}\left(\epsilon^{2}\right) .
$$

Now we define multiplicative renormalization constant

$$
A_{0}=A(\sigma)\left(1+2 \epsilon a+\mathcal{O}\left(\epsilon^{2}\right)\right)
$$

We substitute (2.99) into (2.98) and use the approximation $(1+z)^{\frac{1}{2}}=1+\frac{1}{2} z+\mathcal{O}\left(z^{2}\right)$ to get

$$
Y(x)=-x+\sqrt{A+x^{2}}+\frac{\epsilon a A}{\sqrt{A+x^{2}}}+\epsilon\left(\frac{A_{0} A_{1}}{\sqrt{A_{0}+x^{2}}}+\frac{\sigma-x_{0}}{\sqrt{A+x^{2}}}+\frac{x-\sigma}{\sqrt{A+x^{2}}}\right)+\mathcal{O}\left(\epsilon^{2}\right) .
$$

Hence defining $a=-\frac{\sigma-x_{0}}{A}-A_{1}$ turns out

$$
Y(x)=-x+\sqrt{A+x^{2}}+\epsilon \frac{x-\sigma}{\sqrt{A+x^{2}}}+\mathcal{O}\left(\epsilon^{2}\right) .
$$

Thus applying the RG condition gives

$$
\frac{d A}{d \sigma}=2 \epsilon+\mathcal{O}\left(\epsilon^{2}\right) \Rightarrow A(\sigma)=2 \epsilon \sigma+c+\mathcal{O}\left(\epsilon^{2} \sigma\right)
$$

By setting $\sigma=x$ and $y=\frac{Y}{\epsilon}$ and by using the boundary condition, we surprisingly obtain

$$
y=-\frac{x}{\epsilon}+\sqrt{\frac{x^{2}}{\epsilon^{2}}+\frac{2(x+1)}{\epsilon}+4}+\mathcal{O}\left(\epsilon^{2} \sigma\right) .
$$

Comparing this result to (2.95) implies that the second order RG must vanish. This result will be confirmed in chapter 3. (See (4.33)) 


\section{Chapter 3}

\section{A Rigorous Discussion of the RG Method}

In this chapter we will be discussing the RG method applied to a perturbed autonomous system of differential equations (i.e. contains a small parameter) in a rigorous fashion, see [15].

Most of examples in the previous chapter (and examples in $[11,12]$ ) can be written as the following system

$$
\left\{\begin{array}{l}
\frac{d u}{d \tau}+\frac{1}{\epsilon} A u=F(u), \quad \epsilon \ll 1 \\
u\left(\frac{\tau_{0}}{\epsilon}\right)=u_{0},
\end{array}\right.
$$

such that $A$ is diagonal matrix, say $A=\operatorname{diag}(\Lambda)$, where $\Lambda=\left(\lambda_{1}, \lambda_{2}, \ldots, \lambda_{n}\right), u(t)=$ $\left(u_{1}(t) u_{2}(t) \ldots u_{n}(t)\right)^{T} \in \mathbb{R}^{n}$ and $F(u)$ is a multivariate polynomial with vector coefficients

$$
F(u)=\sum_{\alpha} C_{\alpha} u^{\alpha},
$$

where $\alpha=\left(\alpha_{1}, \alpha_{2}, \ldots, \alpha_{n}\right), C_{\alpha}=\left(C_{\alpha, 1} C_{\alpha, 2} \ldots C_{\alpha, n}\right)^{T}$ and $u^{\alpha}=u_{1}^{\alpha_{1}} u_{2}^{\alpha_{2}} \ldots u_{n}^{\alpha_{n}}$. Also the set $\left\{\alpha \in \mathbb{N}^{n} ; C_{\alpha} \neq 0\right\}$, the set of powers of the polynomial $F$, is finite.

\subsection{The Procedure of the RG Method}

To begin with, we use the change variable $t=\frac{\tau}{\epsilon}$. Hence 


$$
\left\{\begin{array}{l}
\frac{d u}{d t}+A u=\epsilon F(u), \quad \epsilon \ll 1 \\
u\left(t_{0}\right)=u_{0} .
\end{array}\right.
$$

This change of variable is vital to maintain the effect of $\frac{d u}{d t}$ in the approximation.

We now apply the RG procedure discussed in the previous chapter to this system of equations. We proceed in five steps:

\section{- First Step}

First of all, we postulate a naive expansion for the solution of the system (3.2)

$$
u(t)=u^{(0)}(t)+\epsilon u^{(1)}(t)+\epsilon^{2} u^{(2)}(t)+\mathcal{O}\left(\epsilon^{3}\right) .
$$

We substitute (3.3) into (3.2) and then equate the coefficients of $\epsilon$ 's order by order. Now if $D F_{u^{(j)}}$ is the matrix of partial derivatives with respect to $u^{(j)}$, for $j \geq 0$, we obtain

$$
\begin{aligned}
& \frac{d u^{(0)}}{d t}+A u^{(0)}=0, \\
& \frac{d u^{(1)}}{d t}+A u^{(1)}=F\left(u^{(0)}\right), \\
& \frac{d u^{(2)}}{d t}+A u^{(2)}=\left(D F_{u^{(0)}}\right)\left(u^{(1)}\right),
\end{aligned}
$$

and so on. Note that since we are interested only in the first order RG method, we only solve (3.4a) and (3.4b). The general solutions to (3.4a) and (3.4b) are

$$
\begin{aligned}
& u^{(0)}=e^{-A\left(t-t_{0}\right)} v_{0}, \\
& u^{(1)}=e^{-A\left(t-t_{0}\right)} v_{1}+e^{-A\left(t-t_{0}\right)} \int_{t_{0}}^{t} e^{A\left(s-t_{0}\right)} F\left(e^{-A\left(s-t_{0}\right)} v_{0}\right) d s,
\end{aligned}
$$

where $v_{0}, v_{1} \in \mathbb{R}^{n}$ are integral constants. 
Hence

$$
\begin{aligned}
u(t)=u^{(0)}(t)+\epsilon & u^{(1)}(t)+\mathcal{O}\left(\epsilon^{2}\right) \\
& =e^{-A\left(t-t_{0}\right)}\left(v_{0}+\epsilon v_{1}+\epsilon \int_{t_{0}}^{t} e^{A\left(s-t_{0}\right)} F\left(e^{-A\left(s-t_{0}\right)} v_{0}\right) d s\right)+\mathcal{O}\left(\epsilon^{2}\right) .
\end{aligned}
$$

Before introducing the next steps of RG method, we make a few simplifications.

Note that we can ignore the integral constant of $u^{(1)}$; since by defining the vector $\tilde{v}_{0}:=v_{0}+\epsilon v_{1}$, we find

$$
\begin{gathered}
\tilde{v}_{0}+\epsilon \int_{t_{0}}^{t} e^{A\left(t-t_{0}\right)} F\left(e^{-A\left(s-t_{0}\right)} \tilde{v}_{0}-\epsilon e^{-A\left(t-t_{0}\right)} v_{1}\right) d s=\tilde{v}_{0}+ \\
\epsilon \int_{t_{0}}^{t} e^{A\left(s-t_{0}\right)} F\left(e^{-A\left(s-t_{0}\right)} \tilde{v}_{0}\right) d s+\mathcal{O}\left(\epsilon^{2}\right) .
\end{gathered}
$$

Here we have used properties of multivariate polynomial $F$.

For ease of notation, we write

$$
u(t)=e^{-A\left(t-t_{0}\right)}\left(v_{0}+\epsilon \int_{t_{0}}^{t} e^{A\left(s-t_{0}\right)} F\left(e^{-A\left(s-t_{0}\right)} v_{0}\right) d s\right)+\mathcal{O}\left(\epsilon^{2}\right) .
$$

In addition, we can use the decomposition $F\left(v_{0}\right)=R\left(v_{0}\right)+Q\left(v_{0}\right)$, where

$$
\begin{aligned}
& R\left(v_{0}\right)=\sum_{i=1}^{n}\left(\sum_{\Lambda_{\alpha, i}=0} C_{\alpha, i} v_{0}^{\alpha}\right) e_{i} \\
& Q\left(v_{0}\right)=\sum_{i=1}^{n}\left(\sum_{\Lambda_{\alpha, i} \neq 0} C_{\alpha, i} v_{0}^{\alpha}\right) e_{i}
\end{aligned}
$$

such that $\left\{e_{i}\right\}_{i=1}^{n}$ is the standard basis of $\mathbb{R}^{n}$ and $\Lambda_{\alpha, i}=\lambda_{i}-\sum_{j=1}^{n} \lambda_{j} \alpha_{j}$.

We say there is a resonance if $\Lambda_{\alpha, i}=0$ for some $\alpha$ and $i \in\{1,2, \ldots, n\}$ such that $C_{\alpha, i} \neq 0$. If such $\alpha$ and $i$ exist, $C_{\alpha, i} v_{0}^{\alpha}$ is called a resonant term, otherwise it is called a non-resonant term.

We claim that

$$
e^{\left(s-t_{0}\right) A} F\left(e^{-\left(s-t_{0}\right) A} v_{0}\right)=R\left(v_{0}\right)+Q\left(s, v_{0}\right)
$$


where

$$
Q\left(s, v_{0}\right)=\sum_{i=1}^{n}\left(\sum_{\Lambda_{\alpha, i} \neq 0} C_{\alpha, i} e^{\left(s-t_{0}\right) \Lambda_{\alpha, i}} v_{0}^{\alpha}\right) e_{i} .
$$

According to the relation $\left(e^{t A} x\right)^{\alpha}=e^{<\Lambda, \alpha>t} x^{\alpha}$, for every $t \in \mathbb{R}$ and for any vector $x$, we have

$$
\begin{aligned}
R\left(e^{-\left(s-t_{0}\right) A} v_{0}\right) & =\sum_{i=1}^{n}\left(\sum_{\Lambda_{\alpha, i}=0} C_{\alpha, i}\left(e^{-\left(s-t_{0}\right) A} v_{0}\right)^{\alpha}\right) e_{i}=\sum_{i=1}^{n}\left(\sum_{\Lambda_{\alpha, i}=0} C_{\alpha, i} e^{-\left(s-t_{0}\right)<\Lambda, \alpha>} v_{0}^{\alpha}\right) e_{i}= \\
& \sum_{i=1}^{n}\left(\sum_{\Lambda_{\alpha, i}=0} C_{\alpha, i} e^{-\lambda_{i}\left(s-t_{0}\right)} v_{0}^{\alpha}\right) e_{i}=\sum_{i=1}^{n}\left(\sum_{\Lambda_{\alpha, i}=0} C_{\alpha, i} v_{0}^{\alpha}\right) e^{-\lambda_{i}\left(s-t_{0}\right)} e_{i} .
\end{aligned}
$$

Hence

$$
\begin{aligned}
& e^{\left(s-t_{0}\right) A} R\left(e^{-\left(s-t_{0}\right) A} v_{0}\right)=e^{\left(s-t_{0}\right) A} \sum_{i=1}^{n}\left(\sum_{\Lambda_{\alpha, i}=0} C_{\alpha, i} v_{0}^{\alpha}\right) e^{-\lambda_{i}\left(s-t_{0}\right)} e_{i}= \\
& \sum_{i=1}^{n}\left(\sum_{\Lambda_{\alpha, i}=0} C_{\alpha, i} v_{0}^{\alpha}\right) e^{-\lambda_{i}\left(s-t_{0}\right)} e^{\left(s-t_{0}\right) A} e_{i}=\sum_{i=1}^{n}\left(\sum_{\Lambda_{\alpha, i}=0} C_{\alpha, i} v_{0}^{\alpha}\right) e^{-\lambda_{i}\left(s-t_{0}\right)} e^{\lambda_{i}\left(s-t_{0}\right)} e_{i}=R\left(v_{0}\right) .
\end{aligned}
$$

For the other part,

$$
Q\left(e^{-\left(s-t_{0}\right) A} v_{0}\right)=\sum_{i=1}^{n}\left(\sum_{\Lambda_{\alpha, i} \neq 0} C_{\alpha, i}\left(e^{-\left(s-t_{0}\right) A} v_{0}\right)^{\alpha}\right) e_{i}=\sum_{i=1}^{n}\left(\sum_{\Lambda_{\alpha, i} \neq 0} C_{\alpha, i} e^{-\left(s-t_{0}\right)<\Lambda, \alpha>} v_{0}^{\alpha}\right) e_{i}
$$

Hence

$$
\begin{gathered}
e^{\left(s-t_{0}\right) A} Q\left(e^{-\left(s-t_{0}\right) A} v_{0}\right)=\sum_{i=1}^{n}\left(\sum_{\Lambda_{\alpha, i} \neq 0} C_{\alpha, i} e^{-<\Lambda, \alpha>\left(s-t_{0}\right)} v_{0}^{\alpha}\right) e^{\left(s-t_{0}\right) A} e_{i}= \\
\sum_{i=1}^{n}\left(\sum_{\Lambda_{\alpha, i} \neq 0} C_{\alpha, i} e^{-\left(s-t_{0}\right)<\Lambda, \alpha>} v_{0}^{\alpha}\right) e^{\left(s-t_{0}\right) \lambda_{i}} e_{i}=\sum_{i=1}^{n}\left(\sum_{\Lambda_{\alpha, i} \neq 0} C_{\alpha, i} e^{\left(s-t_{0}\right) \Lambda_{\alpha, i}} v_{0}^{\alpha}\right) e_{i} \neq Q\left(v_{0}\right) .
\end{gathered}
$$

According to the definition of a resonant term, we name $R\left(v_{0}\right)$ the resonant part of $F$ and $Q\left(s, v_{0}\right)$ the non-resonant part of $F$.

Therefore

$$
u(t)=e^{-A\left(t-t_{0}\right)}\left(v_{0}+\epsilon \int_{t_{0}}^{t} R\left(v_{0}\right) d s+\epsilon \int_{t_{0}}^{t} Q\left(s, v_{0}\right) d s\right)+\mathcal{O}\left(\epsilon^{2}\right) .
$$


Using (3.7) and integrating term by term imply

$$
\begin{aligned}
& u(t)=e^{-A\left(t-t_{0}\right)}\left(v_{0}+\epsilon\left(t-t_{0}\right) R\left(v_{0}\right)+\right. \\
& \left.\epsilon \sum_{i=1}^{n}\left(\sum_{\Lambda_{\alpha, i} \neq 0} \frac{C_{\alpha, i}}{\Lambda_{\alpha, i}}\left(e^{\left(t-t_{0}\right) \Lambda_{\alpha, i}}-1\right) v_{0}^{\alpha}\right) e_{i}\right)+\mathcal{O}\left(\epsilon^{2}\right) .
\end{aligned}
$$

- Second Step

The second step is to make the change of variable

$$
W_{0}=e^{t_{0} A}\left(v_{0}-\epsilon \sum_{i=1}^{n}\left(\sum_{\Lambda_{\alpha, i} \neq 0} \frac{C_{\alpha, i}}{\Lambda_{\alpha, i}} v_{0}^{\alpha}\right) e_{i}\right)
$$

to get

$$
u(t)=e^{-t A}\left(W_{0}+\epsilon\left(t-t_{0}\right) R\left(W_{0}\right)+\epsilon \sum_{i=1}^{n}\left(\sum_{\Lambda_{\alpha, i} \neq 0} \frac{C_{\alpha, i}}{\Lambda_{\alpha, i}} e^{t \Lambda_{\alpha, i}} W_{0}^{\alpha}\right) e_{i}\right)+\mathcal{O}\left(\epsilon^{2}\right)
$$

- Third Step

$\epsilon\left(t-t_{0}\right) R\left(W_{0}\right)$ is a secular term since it causes the expansion to break down for $t \sim \frac{1}{\epsilon}$. This immediately turns out that the resonant part of the multivariate polynomial $F$ results in the secularities. As the third step, we add and subtract a new parameter, say $\sigma$, between $t$ and $t_{0}$ and we split the secular term to obtain

$$
\begin{aligned}
& u(t)=e^{-t A}\left(W_{0}+\epsilon\left(\sigma-t_{0}\right) R\left(W_{0}\right)\right.+\epsilon(t-\sigma) R\left(W_{0}\right)+ \\
&\left.\epsilon \sum_{i=1}^{n}\left(\sum_{\Lambda_{\alpha, i} \neq 0} \frac{C_{\alpha, i}}{\Lambda_{\alpha, i}} e^{t \Lambda_{\alpha, i}} W_{0}^{\alpha}\right) e_{i}\right)+\mathcal{O}\left(\epsilon^{2}\right) .
\end{aligned}
$$

- Fourth Step

Let us define

$$
V(\sigma)=W_{0}+\epsilon\left(\sigma-t_{0}\right) R\left(W_{0}\right)+\mathcal{O}\left(\epsilon^{2}\right)
$$


Inserting this into (3.10) gives

$$
\begin{aligned}
& u(t)=e^{-t A}(V(\sigma)+\epsilon(t-\sigma) R(V(\sigma))+ \\
& \left.\quad \epsilon \sum_{i=1}^{n}\left(\sum_{\Lambda_{\alpha, i} \neq 0} \frac{C_{\alpha, i}}{\Lambda_{\alpha, i}} e^{t \Lambda_{\alpha, i}} V^{\alpha}(\sigma)\right) e_{i}\right)+\mathcal{O}\left(\epsilon^{2}\right) .
\end{aligned}
$$

Note that changes of variables in (4.5), (3.11) and (3.6) together are equivalent to defining a renormalization constant in the previous chapter.

\section{- Fifth Step}

In the previous chapter, we used the fact that the naive perturbative expansion to the equation must be independent of the artificially added parameter, or $\frac{\partial u}{\partial \sigma}=\mathcal{O}\left(\epsilon^{2}\right)$. We call this equality the RG condition. Nevertheless, there are other ways of deriving the $R G$ equation which are presented in the remarks 3.4 and 3.5. Now by taking the derivative from both sides of (3.12) with respect to $\sigma$ we find

$$
\begin{aligned}
\frac{\partial u}{\partial \sigma}=e^{-t A}\left(\frac{d V(\sigma)}{d \sigma}-\epsilon R(V(\sigma))+\epsilon\right. & (t-\sigma)\left(D_{V}\right)(R) \frac{d V(\sigma)}{d \sigma}+ \\
& \left.\quad \sum_{i=1}^{n}\left(\sum_{\Lambda_{\alpha, i} \neq 0} \frac{C_{\alpha, i}}{\Lambda_{\alpha, i}} e^{t \Lambda_{\alpha, i}} \frac{d V^{\alpha}(\sigma)}{d \sigma}\right) e_{i}\right)+\mathcal{O}\left(\epsilon^{2}\right),
\end{aligned}
$$

where $D_{V}$ is the matrix of partial derivatives with respect to $V$.

Note that

$$
\begin{aligned}
\frac{d V^{\alpha}}{d \sigma}=\alpha_{1} \frac{d V_{1}}{d \sigma} V_{1}^{\alpha_{1}-1} V_{2}^{\alpha_{2}} \ldots V_{n}^{\alpha_{n}}+\alpha_{2} V_{1}^{\alpha_{1}} \frac{d V_{2}}{d \sigma} V_{2}^{\alpha_{2}-1} \ldots V_{n}^{\alpha_{n}}+\ldots+ \\
\alpha_{n} V_{1}^{\alpha_{1}} V_{2}^{\alpha_{2}} \ldots \frac{d V_{n}}{d \sigma} V_{n}^{\alpha_{n}-1}=\sum_{i=1}^{n} \alpha_{i} \frac{d V_{i}}{d \sigma} \frac{V^{\alpha}}{V_{i}},
\end{aligned}
$$

where $V=V(\sigma)$.

On the other hand, $\frac{d V(\sigma)}{d \sigma}=\mathcal{O}(\epsilon)$ (and then $\frac{d V_{i}(\sigma)}{d \sigma}=\mathcal{O}(\epsilon)$ ). Hence

$$
e^{-t A}\left(\frac{d V(\sigma)}{d \sigma}-\epsilon R(V(\sigma))\right)=\mathcal{O}\left(\epsilon^{2}\right)
$$


Therefore, by setting $\sigma=t$, the $\mathrm{RG}$ equation reads

$$
\left\{\begin{array}{l}
\frac{d V}{d t}(t)=\epsilon R(V(t))+\mathcal{O}\left(\epsilon^{2}\right), \\
V\left(t_{0}\right)=W_{0}
\end{array}\right.
$$

where

$$
W_{0}=e^{t_{0} A}\left(u_{0}-\epsilon \sum_{i=1}^{n}\left(\sum_{\Lambda_{\alpha, i} \neq 0} \frac{C_{\alpha, i}}{\Lambda_{\alpha, i}} u_{0}^{\alpha}\right) e_{i}\right) .
$$

Now since $t=\frac{\tau}{\epsilon}$, the solution to the system (3.1) is

$$
u(\tau)=e^{-\frac{\tau}{\epsilon} A} V\left(\frac{\tau}{\epsilon}\right)+\epsilon \sum_{i=1}^{n}\left(\sum_{\Lambda_{\alpha, i} \neq 0} \frac{C_{\alpha, i}}{\Lambda_{\alpha, i}} e^{-\frac{\tau}{\epsilon}<\Lambda, \alpha>} V^{\alpha}\left(\frac{\tau}{\epsilon}\right)\right) e_{i}+\mathcal{O}\left(\epsilon^{2}\right),
$$

where

$$
\left\{\begin{array}{l}
\frac{d V}{d \tau}\left(\frac{\tau}{\epsilon}\right)=R\left(V\left(\frac{\tau}{\epsilon}\right)\right)+\mathcal{O}\left(\epsilon^{2}\right), \\
V\left(\frac{\tau_{0}}{\epsilon}\right)=W_{0}
\end{array}\right.
$$

and

$$
W_{0}=e^{\frac{\tau_{0}}{\epsilon} A}\left(u_{0}-\epsilon \sum_{i=1}^{n}\left(\sum_{\Lambda_{\alpha, i} \neq 0} \frac{C_{\alpha, i}}{\Lambda_{\alpha, i}} u_{0}^{\alpha}\right) e_{i}\right)
$$

\subsection{Estimate the Error of RG Approximation}

In this section we are going to prove that the $\mathrm{RG}$ approximation converges to the solution of the system for small values of $\epsilon$, see [15]. To do this, we define $w(t)=$ $\tilde{u}(t)-u(t)$, where $u(t)$ satisfies the system of differential equations (3.1) and

$$
\tilde{u}(t):=e^{-t A}\left(V(t)+\epsilon \sum_{i=1}^{n}\left(\sum_{\Lambda_{\alpha, i} \neq 0} \frac{C_{\alpha, i}}{\Lambda_{\alpha, i}} e^{t \Lambda_{\alpha, i}} V^{\alpha}(t)\right) e_{i}\right),
$$

is the first order RG approximation and $V(t)$ is the solution to the $\mathrm{RG}$ equation (system).

To show that the RG method's outcome approaches the exact solution, it is sufficient to show that $\|w(t)\| \leq C \epsilon$, for some constant $C$ independent of $\epsilon$. Here we only 
consider two cases for eigenvalues of the diagonalizable matrix $A$ and the solution to the RG equation:

CASE I. There exist $T>0$ and $K>0$ such that

$$
\begin{aligned}
\left\|e^{t A}\right\| & \leq 1 \quad \forall t \in \mathbb{R} \\
\|V(t)\| & \leq K, \quad \forall t-t_{0} \in\left(-\frac{T}{\epsilon}, \frac{T}{\epsilon}\right) .
\end{aligned}
$$

Since $\left\|e^{t A}\right\|=\max _{i \in\{1,2, \ldots, n\}}\left\{e^{t\left(R e \lambda_{i}\right)}\right\}$ and the inequality is satisfied for every $t \in$ $\mathbb{R}$, then the real parts of all eigenvalues have to be zero. Hence $\lambda_{j}$ is zero. In this case, the solution to the system of equations (3.1) has an oscillatory behavior. The Rayleigh Oscillator equation (2.1) is of this type.

CASE II. There exist two constants, $K>0$ and $k>0$ such that:

$$
\begin{array}{r}
\operatorname{Re} \lambda_{i} \geq k, i=1,2, \ldots, n \\
\left\|e^{-t A} V(t)\right\| \leq K, \forall t \geq t_{0} .
\end{array}
$$

In this case, the solution to (3.1), has dissipation behavior with exponential factor(s), when $\epsilon \rightarrow 0+$.

In Theorem 1 and Theorem 2, we will study the autonomous system of differential equations (3.1) in CASE I and CASE II, respectively.

For ease of notation, we set

$$
\begin{aligned}
M(t) & =\sum_{i=1}^{n}\left(\sum_{\Lambda_{\alpha, i} \neq 0} \frac{C_{\alpha, i}}{\Lambda_{\alpha, i}} e^{-t<\Lambda, \alpha>} V^{\alpha}(t)\right) e_{i}, \\
N(t) & =\sum_{i=1}^{n}\left(\sum_{\Lambda_{\alpha, i} \neq 0} \frac{C_{\alpha, i}}{\Lambda_{\alpha, i}} e^{-t<\Lambda, \alpha>} \frac{d}{d t} V^{\alpha}(t)\right) e_{i} .
\end{aligned}
$$

Thus

$$
\tilde{u}(t)=e^{-t A} V(t)+\epsilon M(t) .
$$

Hence, by a straightforward calculations, we find

$$
\frac{d \tilde{u}}{d t}+A \tilde{u}=\epsilon F\left(e^{-t A} V(t)\right)+\epsilon N(t) .
$$


Let $w(t)=u(t)-\tilde{u}(t)$, where $u(t)$ is the actual solution to the system (3.1). Thus

$$
\frac{d w}{d t}+A w=\epsilon\left(F(u)-F\left(e^{-t A} V(t)\right)\right)-\epsilon N(t) .
$$

Hence

$$
\begin{array}{r}
w(t)=e^{-\left(t-t_{0}\right) A} w_{0}+\epsilon e^{-t A} \int_{t_{0}}^{t} e^{s A}\left(F(u(s))-F\left(e^{-s A} V(s)\right)\right) d s- \\
\epsilon e^{-t A} \int_{t_{0}}^{t} e^{s A} N(s) d s .
\end{array}
$$

We will use this formula several times in the next subsections. Also notice that since

$$
V\left(t_{0}\right)=e^{t_{0} A}\left(u_{0}-\epsilon \sum_{i=1}^{n}\left(\sum_{\Lambda_{\alpha, i} \neq 0} \frac{C_{\alpha, i}}{\Lambda_{\alpha, i}} u_{0}^{\alpha}\right) e_{i}\right),
$$

we have

$$
w_{0}=w\left(t_{0}\right)=u\left(t_{0}\right)-\tilde{u}\left(t_{0}\right)=u_{0}-e^{-t_{0} A} V\left(t_{0}\right)-\epsilon M\left(t_{0}\right)=\mathcal{O}(\epsilon)
$$

\subsubsection{Estimate the RG approximation' Error (Oscillatory Case)}

Consider the system

$$
\left\{\begin{array}{l}
\frac{d u}{d t}+A u=\epsilon F(u), \quad \epsilon \ll 1, \\
u\left(t_{0}\right)=u_{0},
\end{array}\right.
$$

where $A$ is a diagonalizable matrix and $F$ is a multivariate polynomial of the form

$$
F(u)=\sum_{\alpha} C_{\alpha} u^{\alpha}
$$

where $\alpha=\left(\alpha_{1}, \alpha_{2}, \ldots, \alpha_{n}\right), \Lambda=\left(\lambda_{1}, \lambda_{2}, \ldots, \lambda_{n}\right), C_{\alpha}=\left(C_{\alpha, 1} C_{\alpha, 2} \ldots C_{\alpha, n}\right)^{T}$ and $u^{\alpha}=$ $u_{1}^{\alpha_{1}} u_{2}^{\alpha_{2}} \ldots u_{n}^{\alpha_{n}}$. Also, for $v$ arbitrary, consider the decomposition $F(v)=R(v)+Q(v)$, where

$$
\begin{aligned}
& R(v)=\sum_{i=1}^{n}\left(\sum_{\Lambda_{\alpha, i}=0} C_{\alpha, i} v^{\alpha}\right) e_{i}, \\
& Q(v)=\sum_{i=1}^{n}\left(\sum_{\Lambda_{\alpha, i} \neq 0} C_{\alpha, i} v^{\alpha}\right) e_{i}
\end{aligned}
$$


where $\Lambda_{\alpha, i}=\lambda_{i}-\sum_{j=1}^{n} \lambda_{j} \alpha_{j}$.

Consider the first order RG approximation

$$
\tilde{u}(t)=e^{-t A} V(t)+\epsilon M(t)
$$

where

$$
M(t)=\sum_{i=1}^{n}\left(\sum_{\Lambda_{\alpha, i} \neq 0} \frac{C_{\alpha, i}}{\Lambda_{\alpha, i}} e^{-t<\Lambda, \alpha>} V^{\alpha}(t)\right) e_{i},
$$

and $V(t)$ is the solution to the RG equation or the system

$$
\left\{\begin{array}{l}
\frac{d V}{d t}=\epsilon R(V(t)) \\
V\left(t_{0}\right)=W_{0}
\end{array}\right.
$$

and

$$
W_{0}=e^{t_{0} A}\left(u_{0}-\epsilon \sum_{i=1}^{n}\left(\sum_{\Lambda_{\alpha, i} \neq 0} \frac{C_{\alpha, i}}{\Lambda_{\alpha, i}} u_{0}^{\alpha}\right) e_{i}\right) .
$$

Then we have the following theorems:

Theorem 3.1. For the system of differential equations

$$
\left\{\begin{array}{l}
\frac{d u}{d t}+A u=\epsilon F(u), \quad \epsilon \ll 1 \\
u\left(t_{0}\right)=u_{0}
\end{array}\right.
$$

suppose

$$
\begin{gathered}
\left\|e^{t A}\right\| \leq 1, \quad \forall t \in \mathbb{R} \\
\|V(t)\| \leq K, \quad \forall t-t_{0} \in\left(-\frac{T}{\epsilon}, \frac{T}{\epsilon}\right),
\end{gathered}
$$

where $V$ is the solution of the system (3.28).

$$
\text { If } w(t)=u(t)-\tilde{u}(t) \text {, where } u(t) \text { is the exact solution to the system (3.29) and } \tilde{u}
$$
is defined in (3.26), then there exists a constant $C$ independent of $\epsilon$ such that

$$
\sup _{-\frac{T}{\epsilon} \leq t \leq \frac{T}{\epsilon}}\|w(t)\| \leq C \epsilon
$$


Proof. Using (3.21), (3.30a) and triangle inequality and some elementary properties of norm of vectors, we get

$$
\|w(t)\| \leq\left\|w_{0}\right\|+\epsilon \int_{t_{0}}^{t}\|G(s)\| d s+\epsilon \int_{t_{0}}^{t}\|N(s)\| d s,
$$

where

$$
\begin{aligned}
& G(s)=F(u)-F\left(e^{-s A} V(s)\right)=F(\tilde{u}+w)-F\left(e^{-s A} V(s)\right), \\
& N(t)=\sum_{i=1}^{n}\left(\sum_{\Lambda_{\alpha, i} \neq 0} \frac{C_{\alpha, i}}{\Lambda_{\alpha, i}} e^{-t<\Lambda, \alpha>} \frac{d}{d t} V^{\alpha}(t)\right) e_{i} .
\end{aligned}
$$

Now by (3.30b), for every $t-t_{0} \in\left(-\frac{T}{\epsilon}, \frac{T}{\epsilon}\right)$ we have

$$
\left|\sum_{\Lambda_{\alpha, i}=0} C_{\alpha, i} V^{\alpha}(t)\right| \leq \sum_{\Lambda_{\alpha, i}=0} c\left|V^{\alpha}(t)\right| \leq m c K^{\alpha_{0}}
$$

such that $m=\operatorname{Card}\left(N_{i}\right)$, where $N_{i}=\left\{\alpha: \Lambda_{\alpha, i}=0, C_{\alpha, i} \neq 0\right\}, \alpha_{0}=\sum_{i=1}^{n} \alpha_{i}$ and $c=\max \left\{\left|C_{\alpha, i}\right| ; \Lambda_{\alpha, i}=0\right\}$.

Notice that since $\|V(t)\|=\sqrt{V_{1}^{2}+\ldots+V_{n}^{2}} \leq K$, then $\left|V_{i}\right| \leq K$ and thus

$$
\left|V^{\alpha}\right|=\left|V_{1}^{\alpha_{1}}\right|\left|V_{2}^{\alpha_{2}}\right| \cdots\left|V_{n}^{\alpha_{n}}\right| \leq K^{\alpha_{0}}
$$

Hence

$$
\|R(V(t))\| \leq c_{0}, \quad \text { uniformly in } t-t_{0} \in\left(-\frac{T}{\epsilon}, \frac{T}{\epsilon}\right),
$$

where $c_{0}=n m c K^{\alpha_{0}}$.

On the other hand, by (3.13), we have

$$
\frac{d V^{\alpha}}{d t}=\sum_{i=1}^{n} \alpha_{i} \frac{d V_{i}}{d t} \frac{V^{\alpha}}{V_{i}}
$$

Also (3.35) and (3.28) imply

$$
\left|\frac{d V_{i}}{d t}\right| \leq\left\|\frac{d V}{d t}\right\|=\epsilon\|R(V(t))\| \leq c_{0} \epsilon
$$

Combining (3.34) and (3.36), for every $t-t_{0} \in\left(-\frac{T}{\epsilon}, \frac{T}{\epsilon}\right)$ we have

$$
\left|\frac{d V^{\alpha}}{d t}\right| \leq \sum_{i=1}^{n}\left|\alpha_{i}\right| \cdot\left|\frac{d V_{i}}{d t}\right| \cdot\left|\frac{V^{\alpha}}{V_{i}}\right| \leq \alpha_{0} c_{0} \epsilon k^{\alpha_{0}-1} .
$$


Therefore

$$
\left|\frac{d V^{\alpha}}{d t}\right| \leq c_{1} \epsilon, \quad \text { uniformly in } t-t_{0} \in\left(-\frac{T}{\epsilon}, \frac{T}{\epsilon}\right)
$$

where $c_{1}=c_{0} \alpha_{0} K^{\alpha_{0}-1}$.

In addition,

$$
\|N(t)\| \leq c_{2} \epsilon, \quad \text { uniformly in } t-t_{0} \in\left(-\frac{T}{\epsilon}, \frac{T}{\epsilon}\right)
$$

such that $c_{2}=\frac{m^{\prime} n c^{\prime} c_{1}}{\lambda}$, where $c^{\prime}=\max _{\Lambda_{\alpha, i} \neq 0}\left\{\left|C_{\alpha, i}\right|\right\}, m^{\prime}=\operatorname{Card}\left(N_{i}^{c}\right),\left(N_{i}^{c}\right.$ is the complement of $N_{i}$ ) and $\lambda=\min _{\alpha \in N_{i}^{c}}\left\{\left|\Lambda_{\alpha, i}\right|\right\}$. To see why, let $t-t_{0} \in\left(-\frac{T}{\epsilon}, \frac{T}{\epsilon}\right)$ be arbitrary. Now by (3.30a) we have

$$
\left\|\sum_{\Lambda_{\alpha, i} \neq 0} \frac{C_{\alpha, i}}{\Lambda_{\alpha, i}} e^{-t<\Lambda, \alpha>} \frac{d}{d t} V^{\alpha}(t)\right\| \leq \sum_{\Lambda_{\alpha, i} \neq 0} \frac{c^{\prime} c_{1} \epsilon}{\lambda}=\frac{m^{\prime} c^{\prime} c_{1} \epsilon}{\lambda}
$$

Also note that by (3.30a) we have

$$
\left|e^{-t<\Lambda, \alpha>}\right|=\left|e^{-t \lambda_{1} \alpha_{1}}\right| \ldots\left|e^{-t \lambda_{n} \alpha_{n}}\right| \leq 1
$$

Similarly, there is the constant $c_{3}$ independent of $\epsilon$ such that

$$
\|M(t)\| \leq c_{3}, \quad \text { uniformly in } t-t_{0} \in\left(-\frac{T}{\epsilon}, \frac{T}{\epsilon}\right) .
$$

The definition of $\tilde{u}$, the triangle inequality, assumption (3.30b) and the inequality (3.38) turn out that there exists a constant $c_{4}$ independent of $\epsilon$ such that

$$
\begin{aligned}
\|\tilde{u}(t)\| \leq\left\|e^{-t A} V(t)\right\|+\|M(t)\| \leq K+c_{3}= & c_{4}, \\
& \text { uniformly in } t-t_{0} \in\left(-\frac{T}{\epsilon}, \frac{T}{\epsilon}\right) .
\end{aligned}
$$

Using triangle inequality, ( see Mean Value Theorem 6.25, in the Appendix) and 
(3.38), for every $s-t_{0} \in\left(-\frac{T}{\epsilon}, \frac{T}{\epsilon}\right)$, we have

$$
\begin{aligned}
\|G(s)\| & =\left\|F(\tilde{u}(s)+w(s))-F\left(e^{-s A} V(s)\right)\right\| \\
& \leq\|F(\tilde{u}(s)+w(s))-F(\tilde{u}(s))\|+\left\|F(\tilde{u}(s))-F\left(e^{-s A} V(s)\right)\right\| \\
& \leq \sup _{\beta \in[0,1]}\|D F(\tilde{u}(s)+\beta w(s))\| \cdot\|w(s)\| \\
& +\epsilon \sup _{\beta \in[0,1]}\|D F(\tilde{u}(s)+\beta M(s))\| \cdot\|M(s)\| \\
& \leq \sup _{\beta \in[0,1]}\|D F(\tilde{u}(s)+\beta w(s))\| .\|w(s)\|+\epsilon m_{0} c_{3} .
\end{aligned}
$$

Notice that we used the inequality $\frac{\|F(x)-F(y)\|}{\|x-y\|} \leq \sup _{\xi \in[0,1]}\|D F(x+\xi y)\|$ (see the Appendix, Theorem 6.25). Also the existence of $m_{0}>0$ is guaranteed due to the boundedness of the vector function $\tilde{u}(s)+\beta M(s)$ (see the Theorem 6.26).

In summary

$$
\|G(s)\| \leq Z(s)\|w(s)\|+\epsilon c_{5}, \quad s-t_{0} \in\left(-\frac{T}{\epsilon}, \frac{T}{\epsilon}\right),
$$

where

$$
Z(s)=\sup _{\beta \in[0,1]}\|D F(\tilde{u}(s)+\beta w(s))\| .
$$

According to (3.22), there exists $k_{0}>0$ such that $\left\|w_{0}\right\| \leq \epsilon k_{0}$. Thus, by (3.37) and (3.41), we conclude

$$
\begin{array}{r}
\|w(t)\| \leq k_{0} \epsilon+\epsilon \int_{t_{0}}^{t} Z(s)\|w(s)\|+\epsilon c_{5} d s+\epsilon \int_{t_{0}}^{t} c_{2} \epsilon d s= \\
\epsilon \int_{t_{0}}^{t} Z(s)\|w(s)\| d s+\epsilon k_{0}+\left(c_{2}+c_{5}\right) \epsilon^{2} t \leq \\
\epsilon \int_{t_{0}}^{t} Z(s)\|w(s)\| d s+\epsilon k_{0}+\left(c_{2}+c_{5}\right) \epsilon T
\end{array}
$$

for every $t-t_{0} \in\left(-\frac{T}{\epsilon}, \frac{T}{\epsilon}\right)$.

Then

$$
\|w(t)\| \leq c_{6} \epsilon+\epsilon \int_{t_{0}}^{t} Z(s)\|w(s)\| d s
$$


where $c_{6}=k_{0}+\left(c_{2}+c_{5}\right) T$. Hence Gronwall's lemma 6.27 implies

$$
\|w(t)\| \leq c_{6} \epsilon e^{\epsilon \int_{t_{0}}^{t} Z(s) d s}
$$

It follows from the definition of $Z(t)$ (3.42) that its boundedness depends on the boundedness of $w(t)$. We claim that

$$
\|w(t)\| \leq 1, \quad \text { uniformly in } t-t_{0} \in\left(-\frac{T}{\epsilon}, \frac{T}{\epsilon}\right) .
$$

Note that $\{t:\|w(t)\| \leq 1\} \neq \emptyset$. Now we prove the following claim: Let

$$
\|w(t)\| \leq 1, \quad \text { uniformly in } t-t_{0} \in\left(T_{1}, T_{2}\right)
$$

where $\left(T_{1}, T_{2}\right) \varsubsetneqq\left(-\frac{T}{\epsilon}, \frac{T}{\epsilon}\right)$ be the maximal interval in which we have such property.

By using (3.39), (3.47) and the Theorem 6.26 we have

$$
Z(s) \leq m_{1}, \quad \text { uniformly in } s-t_{0} \in\left(T_{1}, T_{2}\right)
$$

Thus 3.45 implies

$$
\|w(t)\| \leq c_{6} \epsilon e^{\epsilon m_{1} t} \leq c_{6} \epsilon e^{m_{1} T}=C \epsilon<1, \quad \text { uniformly in } t-t_{0} \in\left(T_{1}, T_{2}\right) .
$$

where $C=c_{6} e^{m_{1} T}$ (which is independent of $\epsilon$ ).

Since $w(t)$ is a continuous function, by choosing $\epsilon$ to be small enough, we can extend the inequality (3.2.1) for a bigger interval which contains $\left(T_{1}, T_{2}\right)$. This contradicts with the maximality of the interval $\left(T_{1}, T_{2}\right)$ for which $(3.2 .1)$ holds. Thus

$$
\|w(t)\| \leq 1, \quad \text { uniformly in } t-t_{0} \in\left(-\frac{T}{\epsilon}, \frac{T}{\epsilon}\right)
$$

Again, by applying (3.39), (3.47) and (6.26) we have

$$
Z(s) \leq m_{1}, \quad \text { uniformly in } t-t_{0} \in\left(-\frac{T}{\epsilon}, \frac{T}{\epsilon}\right) .
$$

Therefore

$$
\|w(t)\| \leq C \epsilon, \quad \text { uniformly in } t-t_{0} \in\left(-\frac{T}{\epsilon}, \frac{T}{\epsilon}\right)
$$


which implies (3.31).

Remark 3.2. In this case, we have proved that the RG method's approximation to a system whose initial time is $t_{0}$ is valid in the interval $\left[t_{0}, t_{0}+\frac{T}{\epsilon}\right]$, for some $T$. In this remark we want to extend the region of validity of the RG method. Following the proof, we realize that there is a constant $c>1$ independent of $\epsilon$ such that

$$
\|w(t)\|^{2} \leq c\left(\epsilon^{2}+\left\|w\left(t_{0}\right)\right\|^{2}\right),
$$

where $t \in\left[t_{0}, t_{0}+\frac{1}{\epsilon}\right]$. Let $t_{i}=t_{0}+\frac{i}{\epsilon}$. Hence the inequality,

$$
\|w(t)\|^{2} \leq c\left(\epsilon^{2}+\left\|w\left(t_{i-1}\right)\right\|^{2}\right),
$$

holds for $t \in\left[t_{i-1}, t_{i}\right]$. Since $i$ is arbitrary, the inequality is true for the intervals $\left[t_{i-2}, t_{i-1}\right],\left[t_{i-3}, t_{i-2}\right]$ and so forth. Thus for $t \in\left[t_{0}, t_{i}\right]=\left[t_{0}, t_{1}\right] \bigcup\left[t_{1}, t_{2}\right] \bigcup \ldots \bigcup\left[t_{i-1}, t_{i}\right]$,

$$
\begin{gathered}
\|w(t)\|^{2} \leq c\left(\epsilon^{2}+\left\|w\left(t_{i-1}\right)\right\|^{2}\right) \leq c\left(\epsilon^{2}+\left(c\left(\epsilon^{2}+\left\|w\left(t_{i-2}\right)\right\|^{2}\right)\right)\right)= \\
\epsilon^{2}\left(\sum_{j=1}^{2} c^{j}\right)+c^{2}\left\|w\left(t_{i-2}\right)\right\|^{2} \leq \epsilon^{2}\left(\sum_{j=1}^{3} c^{j}\right)+c^{3}\left\|w\left(t_{i-3}\right)\right\|^{2} \leq \ldots \leq \\
\epsilon^{2}\left(\sum_{j=1}^{i} c^{j}\right)+c^{i}\left\|w\left(t_{0}\right)\right\|^{2} \leq\left(\frac{c\left(c^{i}-1\right)}{c-1}\right) \epsilon^{2}+c^{i+2} \epsilon^{2} \leq c^{i+3} \epsilon^{2}
\end{gathered}
$$

If $i$ is a natural number such that $c^{i+3} \leq \epsilon^{-\delta}$, where $0<\delta<1$. Hence $i \leq \frac{\delta|\ln (\epsilon)|}{\ln (c)}-3$. By rounding the left hand side down, we find an appropriate $i$. Hence for some positive constants $C_{1}$ and $C_{2}$ independent of $\epsilon$ we find

$$
\sup _{t \in\left[t_{0}, t_{0}+C_{1} \frac{\delta \mid \ln (\epsilon) !}{\epsilon}\right]}\|w(t)\| \leq C_{2} \epsilon^{1-\frac{\delta}{2}}
$$

\subsubsection{Estimate the RG approximation' Error (Dissipation Case)}

Theorem 3.3. Let $u(t)$ be the solution to the following system of equations

$$
\left\{\begin{array}{l}
\frac{d u}{d t}+A u=\epsilon F(u), \quad \epsilon \ll 1 \\
u\left(t_{0}\right)=u_{0} .
\end{array}\right.
$$


and assume that there exist $K>0$ and $l>0$, such that

$$
\begin{aligned}
& R e \lambda_{i} \geq l, \quad \forall i=1,2, \ldots, n \\
&\left\|e^{-t A} V(t)\right\| \leq K, \quad \forall t \geq t_{0}
\end{aligned}
$$

where $V(t)$ is the solution of the $R G$ equation. Then there exists an $\epsilon$-independent constant $C$ such that

$$
\sup _{t \geq 0}\|w(t)\| \leq C \epsilon
$$

where $w(t)=u(t)-\tilde{u}(t)$ such that $u$ is the exact solution to the system and $\tilde{u}$ is the first order $R G$ approximation in (3.26).

Proof. To begin with, we prove several statements that help us to prove the argument. First of all, referring to (3.8) and (3.9), for every $t \geq t_{0}$ we have

$$
e^{-t A} R(V(t))=R\left(e^{-t A} V(t)\right)=\sum_{i=1}^{n}\left(\sum_{\Lambda_{\alpha, i}=0} C_{\alpha, i}\left(e^{-t A} V(t)\right)^{\alpha}\right) e_{i}
$$

Now (3.54b) implies that

$$
\sup _{t \geq 0}\left\|e^{-t A} R(V(t))\right\| \leq m c K^{\alpha_{0}-1}
$$

where $c=\max \left\{\left|C_{\alpha, i}\right| ; \Lambda_{\alpha, i}=0\right\}, \alpha_{0}=\sum_{i=1}^{n} \alpha_{i}$ and $m=\operatorname{Card}\left(N_{i}\right)$, where $N_{i}=\{\alpha$ : $\left.C_{\alpha, i} \neq 0, \Lambda_{\alpha, i}=0\right\}$. Hence the RG equation (3.28) $\left\|e^{-t A} \frac{d V}{d t}\right\| \leq \tilde{c}_{0} \epsilon$. This inequality and $(3.54 \mathrm{~b})$ give

$$
\begin{gathered}
\left|e^{-\lambda_{i} t} \frac{d V_{i}}{d t}\right| \leq \tilde{c}_{0} \epsilon \\
\left|e^{-\lambda_{i} t} V_{i}\right| \leq K
\end{gathered}
$$




\section{Hence}

$$
\begin{gathered}
\left|e^{-t<\Lambda, \alpha>} \frac{d}{d t} V^{\alpha}\right|=\mid e^{-t \lambda_{1} \alpha_{1}} e^{-t \lambda_{2} \alpha_{2}} \ldots e^{-t \lambda_{n} \alpha_{n}}\left(\alpha_{1} V_{1}^{\prime} V_{1}^{\alpha_{1}-1} V_{2}^{\alpha_{2}} \ldots V_{n}^{\alpha_{n}}+\right. \\
\left.\ldots+\alpha_{n} V_{n}^{\prime} V_{1}^{\alpha_{1}} V_{2}^{\alpha_{2}} \ldots V_{n}^{\alpha_{n}-1}\right)|=| \alpha_{1}\left(e^{-\lambda_{1} t} V_{1}^{\prime}\right)\left(e^{-t \lambda_{1}\left(\alpha_{1}-1\right)} V_{1}^{\alpha_{1}-1}\right)\left(e^{-t \lambda_{2} \alpha_{2}} V_{2}^{\alpha_{2}}\right) \times \\
\ldots \times\left(e^{-t \lambda_{n} \alpha_{n}} V_{n}^{\alpha_{n}}\right)+\ldots+\alpha_{n}\left(e^{-\lambda_{n} t} V_{n}^{\prime}\right)\left(e^{-t \lambda_{1} \alpha_{1}} V_{1}^{\alpha_{1}}\right)\left(e^{-t \lambda_{2} \alpha_{2}} V_{2}^{\alpha_{2}}\right) \ldots\left(e^{-t \lambda_{n}\left(\alpha_{n}-1\right)} V_{n}^{\alpha_{n}-1}\right) \mid \\
=\left|\sum_{i=1}^{n} \alpha_{i}\left(e^{-\lambda_{i} t} V_{i}^{\prime}\right)\left(e^{-\lambda_{1} t} V_{1}\right)^{\alpha_{1}} \ldots\left(e^{-\lambda_{i} t} V_{i}\right)^{\alpha_{i}-1} \ldots\left(e^{-\lambda_{n} t} V_{n}\right)^{\alpha_{n}}\right| \leq \alpha_{0} \tilde{c} K^{\alpha_{0}-1} \epsilon .
\end{gathered}
$$

Thus for every $t \geq t_{0}$, there is a constant $c_{1}$, independent of $\epsilon$ such that

$$
\sup _{t \geq t_{0}}\|N(t)\|=\left\|\sum_{i=1}^{n}\left(\sum_{\Lambda_{\alpha, i} \neq 0} \frac{C_{\alpha, i}}{\Lambda_{\alpha, i}} e^{-t<\Lambda, \alpha>} \frac{d}{d t} V^{\alpha}(t)\right) e_{i}\right\| \leq c_{1} \epsilon .
$$

Also for every $t \geq t_{0}$, we have

$$
\sup _{t \geq t_{0}}\|M(t)\|=\left\|\sum_{i=1}^{n}\left(\sum_{\Lambda_{\alpha, i} \neq 0} C_{\alpha, i}\left(e^{-t A} V(t)\right)^{\alpha}\right) e_{i}\right\| \leq c_{2} \epsilon,
$$

where $c_{2}=m^{\prime} c^{\prime} K^{\alpha_{0}}$, independent of $\epsilon$.

The RG equation (or system) (3.28), (3.58) and using the triangle inequality turn out that there is a constant $c_{3}$, independent of $\epsilon$ such that

$$
\sup _{t \geq t_{0}}\|\tilde{u}(t)\|=\left\|e^{-t A} V(t)+\epsilon M(t)\right\| \leq\left\|e^{-t A} V(t)\right\|+\|\epsilon M(t)\| \leq K+c_{2}=c_{3} .
$$

On the other hand, if

$$
G(s)=F(u)-F\left(e^{-s A} V(s)\right)=F(\tilde{u}+w)-F\left(e^{-s A} V(s)\right),
$$

then by using (3.21) and (3.57), we have

$$
\begin{gathered}
\|w(t)\| \leq\left\|e^{-t A} w_{0}\right\|+\epsilon \int_{t_{0}}^{t}\left\|e^{(s-t) A} G(s)\right\| d s+\epsilon \int_{t_{0}}^{t}\left\|e^{(s-t) A} N(s)\right\| d s \\
\leq e^{-l t}\left\|w_{0}\right\|+\epsilon \int_{t_{0}}^{t} e^{(s-t) l}\|G(s)\| d s+\epsilon \int_{t_{0}}^{t} e^{(s-t) l} c_{1} \epsilon d s \\
\leq\left\|w_{0}\right\|+\epsilon \int_{t_{0}}^{t} e^{(s-t) l}\|G(s)\| d s+\frac{c_{1}}{l} \epsilon^{2} .
\end{gathered}
$$


By doing the same procedure as in (3.40), we conclude that there exists some constant $c_{5}$ such that for every $t \geq t_{0}$,

$$
\|G(t)\| \leq Z(t)\|w(t)\|+c_{5} \epsilon
$$

where

$$
Z(t)=\sup _{\beta \in[0,1]}\|D F(\tilde{u}(t)+\beta w(t))\| .
$$

According to (3.22), there exists a constant $k_{0}$, independent of $\epsilon$, such that $\left\|w_{0}\right\| \leq$ $k_{0} \epsilon$. Hence $(3.60)$ yields

$$
\|w(t)\| \leq c_{6} \epsilon+\epsilon \int_{t_{0}}^{t} e^{(s-t) l} Z(s)\|w(s)\| d s,
$$

where $c_{6}=k_{0}+\frac{1}{l}\left(c_{1}+c_{5}\right)$.

By Gronwall's lemma 6.27, we have

$$
\|w(t)\| \leq c_{6} \epsilon e^{\epsilon e^{-l t} \int_{t_{0}}^{t} e^{l r} Z(r) d r} .
$$

Now let $\left(T_{1}, T_{2}\right) \subset\left[t_{0}, \infty\right)$ be the maximal interval such that for every $t \in\left(T_{1}, T_{2}\right)$, we have

$$
\|w(t)\| \leq 1
$$

By using (3.19), (3.59) and (6.26), there is a positive number $m$, such that

$$
Z(r) \leq m, \quad r \in\left(T_{1}, T_{2}\right)
$$

Thus

$$
\|w(t)\| \leq c_{6} \epsilon e^{\epsilon e^{-l t}\left(\frac{m}{l}\right)\left(e^{l t}-e^{l t_{0}}\right)} \leq c_{6} \epsilon e^{\frac{\epsilon m}{l}}=c_{7} \epsilon<1 \quad t \in\left(T_{1}, T_{2}\right)
$$

where $c_{7}=c_{6} e^{\frac{\epsilon m}{l}}$ is a constant independent of $\epsilon$.

Since $w(t)$ is a continuous function, by choosing $\epsilon$ to be small enough, (3.61) holds for a bigger interval than $\left(T_{1}, T_{2}\right)$. This contradicts with the existence of such maximal interval. Thus

$$
Z(r) \leq m, \quad r \geq t_{0}
$$


By following the same, we find out that there exists a constant $C$ independent of $\epsilon$ such that

$$
\|w(t)\| \leq C \epsilon, \quad t \geq t_{0} .
$$

This proves (3.55).

\subsection{Reproducing the RG Equation}

Remark 3.4. Recall the RG approximation before applying the RG condition:

$$
\begin{aligned}
& u(t)=e^{-t A}(V(\sigma)+\epsilon(t-\sigma) R(V(\sigma))+ \\
& \left.\quad \epsilon \sum_{i=1}^{n}\left(\sum_{\Lambda_{\alpha, i} \neq 0} \frac{C_{\alpha, i}}{\Lambda_{\alpha, i}} e^{t \Lambda_{\alpha, i}} V^{\alpha}(\sigma)\right) e_{i}\right)+\mathcal{O}\left(\epsilon^{2}\right) .
\end{aligned}
$$

The aim is to reproduce the $R G$ equation using an equivalent idea used in the fifth step in section 1. It is enough to observe that $V(\sigma)+\epsilon R(V(\sigma))(t-\sigma)$ resembles two first terms of the Taylor expansion of some function around the point $t=\sigma$. Hence

$$
\left\{\begin{array}{l}
\frac{d V}{d t}(t)=\epsilon R(V(t)), \\
V\left(t_{0}\right)=W_{0}
\end{array}\right.
$$

where

$$
W_{0}=e^{t_{0} A}\left(u_{0}-\epsilon \sum_{i=1}^{n}\left(\sum_{\Lambda_{\alpha, i} \neq 0} \frac{C_{\alpha, i}}{\Lambda_{\alpha, i}} u_{0}^{\alpha}\right) e_{i}\right) .
$$

This is the same as the RG equation obtained in (3.14). This interpretation of the RG condition was proposed by Ziane [15].

Remark 3.5. The RG equation derived in (3.14) can be derived using the fact that the multiplicative renormalization constant, used in the previous chapter, must be free of the artificially added parameter. Recall the naive expansion solution which 
contains a secular term:

$$
\begin{aligned}
u(t)=e^{-t A}\left(W_{0}+\epsilon\left(\sigma-t_{0}\right) R\left(W_{0}\right)\right. & +\epsilon(t-\sigma) R\left(W_{0}\right)+ \\
& \left.\epsilon \sum_{i=1}^{n}\left(\sum_{\Lambda_{\alpha, i} \neq 0} \frac{C_{\alpha, i}}{\Lambda_{\alpha, i}} e^{t \Lambda_{\alpha, i}} W_{0}^{\alpha}\right) e_{i}\right)+\mathcal{O}\left(\epsilon^{2}\right) .
\end{aligned}
$$

Now we define the multiplicative renormalization constant

$$
W_{0}=V(\sigma)+a \epsilon V(\sigma)+\mathcal{O}\left(\epsilon^{2}\right) .
$$

We find $a$ such that the secular term that contains $\left(\sigma-t_{0}\right)$ to be cancelled out. Therefore

$$
W_{0}=V(\sigma)-\epsilon\left(\sigma-t_{0}\right) R(V(\sigma))+\mathcal{O}\left(\epsilon^{2}\right)
$$

By taking derivative with respect to $\sigma$, we obtain

$$
0=\frac{V(\sigma)}{d \sigma}-\epsilon R(V(\sigma))-\epsilon\left(\sigma-t_{0}\right)\left(D_{V(\sigma)}\right)(R) \frac{V(\sigma)}{d \sigma}+\mathcal{O}\left(\epsilon^{2}\right)
$$

where $D_{V}$ is the matrix of partial derivatives with respect to $V$.

Since $\frac{V(\sigma)}{d \sigma}=\mathcal{O}(\epsilon)$, we have

$$
\frac{V(\sigma)}{d \sigma}-\epsilon R(V(\sigma))=\mathcal{O}\left(\epsilon^{2}\right)
$$

Therefore, by setting $\sigma=t$ we find

$$
\left\{\begin{aligned}
\frac{d V}{d t}(t) & =\epsilon R(V(t))+\mathcal{O}\left(\epsilon^{2}\right), \\
V\left(t_{0}\right) & =W_{0}
\end{aligned}\right.
$$

where

$$
W_{0}=e^{t_{0} A}\left(u_{0}-\epsilon \sum_{i=1}^{n}\left(\sum_{\Lambda_{\alpha, i} \neq 0} \frac{C_{\alpha, i}}{\Lambda_{\alpha, i}} u_{0}^{\alpha}\right) e_{i}\right) .
$$

This way of finding the RG equation was proposed by Oono and Nozaki [18]. 
Remark 3.6. Relationship between the RG and Multiple Scale Analysis

In chapter two, we applied the RG method and the multiple scale analysis to two equations, the Rayleigh oscillator and the Mathieu equation. By comparing the results of multiple scale analysis with results of $R G$, we guess that the multiple scale analysis and RG method are equivalent (see [21] for more information about the equivalence of these two methods for ODEs). In this remark, we want to show that, in the case of autonomous system of differential equations, the RG method and the multiple scale analysis produce the identical results. To see this, suppose that the autonomous differential equation

$$
\left\{\begin{array}{l}
\frac{d u}{d t}+A u=\epsilon F(u), \quad \epsilon \ll 1, \\
u\left(t_{0}\right)=u_{0},
\end{array}\right.
$$

is given. As we observed, by postulating the naive perturbative expansion for the solution of this system we find

$$
u(t)=e^{-A\left(t-t_{0}\right)}\left(v_{0}+\epsilon\left(t-t_{0}\right) R\left(v_{0}\right) d s+\epsilon \int_{t_{0}}^{t} Q\left(s, v_{0}\right) d s\right)+\mathcal{O}\left(\epsilon^{2}\right) .
$$

Due to existence of $\left(t-t_{0}\right)$, the naive expansion breaks down. The multiple scale analysis suggests to define the parameter $\sigma=\epsilon\left(t-t_{0}\right)$ and to postulate the following expansion:

$$
u(t)=u^{(0)}(t, \sigma)+\epsilon u^{(1)}(t, \sigma)+\mathcal{O}\left(\epsilon^{2}\right) .
$$

By using the Chain rule we get

$$
\frac{d u}{d t}=\frac{\partial u^{(0)}}{\partial t}+\epsilon \frac{\partial u^{(0)}}{\partial \sigma}+\epsilon \frac{\partial u^{(1)}}{\partial t}+\mathcal{O}\left(\epsilon^{2}\right) .
$$

By substituting this into the system (3.71) we obtain

$$
\frac{\partial u^{(0)}}{\partial t}+\epsilon\left(\frac{\partial u^{(0)}}{\partial \sigma}+\frac{\partial u^{(1)}}{\partial t}\right)+A\left(u^{(0)}+\epsilon u^{(1)}\right)=\epsilon\left(R\left(u^{(0)}\right)+Q\left(u^{(0)}\right)\right)+\mathcal{O}\left(\epsilon^{2}\right),
$$

where $R(u)$ and $Q(u)$ are, respectively, the resonant and non-resonant parts of the multivariate polynomial $F(u)$ defined in (3.24). 
Hence

$$
\begin{aligned}
& \frac{\partial u^{(0)}(t, \sigma)}{\partial t}+A u^{(0)}(t, \sigma)=0 \\
& \frac{\partial u^{(1)}(t, \sigma)}{\partial t}+A u^{(1)}(t, \sigma)=-\frac{\partial u^{(0)}}{\partial \sigma}+R\left(u^{(0)}\right)+Q\left(u^{(0)}\right) .
\end{aligned}
$$

The solutions to (3.72) read

$$
\begin{aligned}
u^{(0)}(t, \sigma) & =e^{-\left(t-t_{0}\right) A} v_{0}(\sigma), \\
u^{(1)}(t, \sigma) & =e^{-\left(t-t_{0}\right) A} v_{1}(\sigma)+e^{-\left(t-t_{0}\right) A} \int_{t_{0}}^{t} e^{\left(s-t_{0}\right) A}\left(-\frac{\partial u^{(0)}(s, \sigma)}{\partial \sigma}+\right. \\
& \left.R\left(u^{(0)}(s, \sigma)\right)+Q\left(u^{(0)}(s, \sigma)\right)\right) d s .
\end{aligned}
$$

Now since $\frac{\partial u^{(0)}(s, \sigma)}{\partial \sigma}=e^{-\left(s-t_{0}\right) A} \frac{d v_{0}(\sigma)}{d \sigma}$, we have

$$
\begin{aligned}
& u(t)=e^{-\left(t-t_{0}\right) A}\left(v_{0}(\sigma)+\epsilon v_{1}(\sigma)+\epsilon \int_{t_{0}}^{t}\left(-\frac{d v_{0}(\sigma)}{d \sigma}+\right.\right. \\
&\left.\left.R\left(v_{0}(\sigma)\right)+e^{\left(s-t_{0}\right) A} Q\left(e^{-\left(s-t_{0}\right) A} v_{0}(\sigma)\right)\right) d s\right)+\mathcal{O}\left(\epsilon^{2}\right) .
\end{aligned}
$$

Since $-\frac{d v_{0}(\sigma)}{d \sigma}+R\left(v_{0}(\sigma)\right)$ does not depend on the integral variable, it can be taken out and causes secularity. Hence it must be removed:

$$
-\frac{d v_{0}(\sigma)}{d \sigma}+R\left(v_{0}(\sigma)\right)=0
$$

Hence by setting $\sigma=\epsilon\left(t-t_{0}\right)$ we find

$$
\frac{d v_{0}(t)}{d t}=\epsilon R\left(v_{0}(t)\right)
$$

In addition, the initial value is $\left.v_{0}(t)\right|_{t=t_{0}}=u\left(t_{0}\right)=u_{0}$. Therefore, for an autonomous system of ordinary differential equations (3.71), the RG method and the multiple scale analysis produce identical results. 


\section{Chapter 4}

\section{Simplified RG Method And The SECOND ORDER RG}

In the previous chapter we learned how we can apply RG method to an autonomous system of ordinary differential equations and why we are certain that RG outcome converges to the solution of the original perturbed differential equation. In this section, we introduce a simplified $R G$ which is in 3 steps [16]. By using this reduced method we then investigate the second order RG approximation.

\subsection{The Simplified Procedure}

Consider the following autonomous system of equations:

$$
\left\{\begin{array}{l}
\dot{u}+A u=\epsilon \sum_{\alpha, i} C_{\alpha, i} u^{\alpha} e_{i}, \\
u\left(t_{0}\right)=u_{0}
\end{array}\right.
$$

where $A$ is a diagonal matrix, $u(t)=\left(u_{1}(t) \ldots u_{n}(t)\right)^{T} \in \mathbb{R}^{n}$ is the solution to the system (4.1), $\alpha=\left(\alpha_{1}, \alpha_{2}, \ldots, \alpha_{n}\right)$ and $u^{\alpha}=u_{1}^{\alpha_{1}} u_{2}^{\alpha_{2}} \ldots u_{n}^{\alpha_{n}}$.

We are going to apply the CGO-RG method, as described in the second chapter, to the system (4.1). First, we postulate the naive perturbative expansion for the solution of the system

$$
u(t)=u^{(0)}(t)+\epsilon u^{(1)}(t)+\mathcal{O}\left(\epsilon^{2}\right) .
$$


Hence we find

$$
\begin{aligned}
& u^{(0)}(t)=e^{-A\left(t-t_{0}\right)} w_{0}, \\
& u^{(1)}(t)=e^{-A\left(t-t_{0}\right)}\left(\left(t-t_{0}\right) \sum_{\Lambda_{\alpha, i}=0} C_{\alpha, i} w_{0}^{\alpha} e_{i}+\sum_{\Lambda_{\alpha, i} \neq 0} \frac{C_{\alpha, i}}{\Lambda_{\alpha, i}}\left(e^{\left(t-t_{0}\right) \Lambda_{\alpha, i}}-1\right) w_{0}^{\alpha} e_{i}\right),
\end{aligned}
$$

where $w_{0}$ is an integral vector constant and $\Lambda_{\alpha, i}=\lambda_{i}-\sum_{j=1}^{n} \lambda_{j} \alpha_{j}$. Note that $\Lambda_{\alpha, i}=0$ if $\sum_{j=1}^{n} \lambda_{j} \alpha_{j}=\lambda_{i}$, where $\lambda_{i}$ is the $i$ th eigenvalues of the matrix coefficients of the linear part of the system.

Thus the solution up to and including the first order is

$$
\begin{aligned}
u(t)=e^{-A\left(t-t_{0}\right)}\left(w_{0}+\epsilon\left(t-t_{0}\right)\right. & \sum_{\Lambda_{\alpha, i}=0} C_{\alpha, i} w_{0}^{\alpha} e_{i}+ \\
& \left.\epsilon \sum_{\Lambda_{\alpha, i} \neq 0} \frac{C_{\alpha, i}}{\Lambda_{\alpha, i}}\left(e^{\left(t-t_{0}\right) \Lambda_{\alpha, i}}-1\right) w_{0}^{\alpha} e_{i}\right)+\mathcal{O}\left(\epsilon^{2}\right) .
\end{aligned}
$$

We use the change of variable

$$
v_{0}=e^{t_{0} A}\left(w_{0}-\epsilon \sum_{i=1}^{n}\left(\sum_{\Lambda_{\alpha, i} \neq 0} \frac{C_{\alpha, i}}{\Lambda_{\alpha, i}} w_{0}^{\alpha}\right) e_{i}\right),
$$

to get

$$
u(t)=e^{-A t}\left(v_{0}+\epsilon\left(t-t_{0}\right) \sum_{\Lambda_{\alpha, i}=0} C_{\alpha, i} v_{0}^{\alpha} e_{i}+\epsilon \sum_{\Lambda_{\alpha, i} \neq 0} \frac{C_{\alpha, i}}{\Lambda_{\alpha, i}} e^{t \Lambda_{\alpha, i}} v_{0}^{\alpha} e_{i}\right)+\mathcal{O}\left(\epsilon^{2}\right) .
$$

Now we define the new parameter and split the equation (4.6)

$$
\begin{aligned}
& u(t)=e^{-A t}\left(v_{0}+\epsilon\left(\sigma-t_{0}\right) \sum_{\Lambda_{\alpha, i}=0} C_{\alpha, i} v_{0}^{\alpha} e_{i}+\right. \\
& \left.\quad \epsilon(t-\sigma) \sum_{\Lambda_{\alpha, i}=0} C_{\alpha, i} v_{0}^{\alpha} e_{i}+\epsilon \sum_{\Lambda_{\alpha, i} \neq 0} \frac{C_{\alpha, i}}{\Lambda_{\alpha, i}} e^{t \Lambda_{\alpha, i}} v_{0}^{\alpha} e_{i}\right)+\mathcal{O}\left(\epsilon^{2}\right) .
\end{aligned}
$$

To absorb the terms containing $\left(\sigma-t_{0}\right)$, we consider the following renormalization constant

$$
V(\sigma)=v_{0}+\epsilon\left(\sigma-t_{0}\right) \sum_{\Lambda_{\alpha, i}=0} C_{\alpha, i} v_{0}^{\alpha} e_{i}+\mathcal{O}\left(\epsilon^{2}\right)
$$


Hence

$$
\begin{gathered}
u(t)=e^{-A t}\left(V(\sigma)+\epsilon(t-\sigma) \sum_{\Lambda_{\alpha, i}=0} C_{\alpha, i} V(\sigma)^{\alpha} e_{i}+\right. \\
\left.\epsilon \sum_{\Lambda_{\alpha, i} \neq 0} \frac{C_{\alpha, i}}{\Lambda_{\alpha, i}} e^{t \Lambda_{\alpha, i}} V(\sigma)^{\alpha} e_{i}\right)+\mathcal{O}\left(\epsilon^{2}\right) .
\end{gathered}
$$

What are we observing here? We are seeing that (4.6) and (4.7) are identical except for the initial value which is constant in (4.6) but depends on $\sigma$ in (4.7). This variable, $\sigma$, has a freedom which allows us to have the RG condition. In the simplified $\mathrm{RG}$ method, the idea is to have this freedom from the beginning. Let the initial time of the given system be $T_{0}$. By replacing $T_{0}$ by an arbitrary initial time, say $t_{0}$ where $T_{0} \leq t_{0} \leq t$, and by postulating the naive perturbative expansion for the solution of the new system (the system having the initial time $t_{0}$ ), we find

$$
\begin{aligned}
u(t)=e^{-A\left(t-t_{0}\right)}\left(w\left(t_{0}\right)+\epsilon\left(t-t_{0}\right)\right. & \sum_{\Lambda_{\alpha, i}=0} C_{\alpha, i} w\left(t_{0}\right)^{\alpha} e_{i}+ \\
& \left.\epsilon \sum_{\Lambda_{\alpha, i} \neq 0} \frac{C_{\alpha, i}}{\Lambda_{\alpha, i}}\left(e^{\left(t-t_{0}\right) \Lambda_{\alpha, i}}-1\right) w\left(t_{0}\right)^{\alpha} e_{i}\right)+\mathcal{O}\left(\epsilon^{2}\right) .
\end{aligned}
$$

Since $t_{0}$ is arbitrary between $T_{0}$ and $t$, we are allowed to differentiate with respect to $t_{0}$. Hence in the simplified RG method, the RG condition is $\left.\frac{\partial u}{\partial t_{0}}\right|_{t_{0}=t}=\mathcal{O}\left(\epsilon^{2}\right)$.

Before applying the RG condition, we make the following change of variable to absorb homogeneous solution of $u^{(1)}$ :

$$
w\left(t_{0}\right)=W\left(t_{0}\right)+\epsilon \sum_{\Lambda_{\alpha, i} \neq 0} \frac{C_{\alpha, i}}{\Lambda_{\alpha, i}} W\left(t_{0}\right)^{\alpha} e_{i} \cdot{ }^{1}
$$

\footnotetext{
${ }^{1}$ Note that if $W$ is bounded, this change of variable is a near-identity change of variable.
} 
Hence we find

$$
\begin{aligned}
u(t)=e^{-A\left(t-t_{0}\right)} W+\epsilon\left(t-t_{0}\right) e^{-A\left(t-t_{0}\right)} & \sum_{\Lambda_{\alpha, i}=0} C_{\alpha, i} W^{\alpha} e_{i}+ \\
\epsilon e^{-A\left(t-t_{0}\right)} & \sum_{\Lambda_{\alpha, i} \neq 0} \frac{C_{\alpha, i}}{\Lambda_{\alpha, i}} e^{\left(t-t_{0}\right) \Lambda_{\alpha, i}} W^{\alpha} e_{i}+\mathcal{O}\left(\epsilon^{2}\right),
\end{aligned}
$$

where, for convenience, we used $W$ instead of $W\left(t_{0}\right)$.

Now we apply the RG condition $\left.\frac{\partial u}{\partial t_{0}}\right|_{t_{0}=t}=\mathcal{O}\left(\epsilon^{2}\right)$ :

$$
\begin{gathered}
\frac{d u}{d t_{0}}=A e^{-A\left(t-t_{0}\right)} W+e^{-A\left(t-t_{0}\right)} \frac{d W}{d t_{0}}-\epsilon e^{-A\left(t-t_{0}\right)} \sum_{\Lambda_{\alpha, i}=0} C_{\alpha, i} W^{\alpha} e_{i} \\
+\epsilon\left(t-t_{0}\right) A e^{-A\left(t-t_{0}\right)} \sum_{\Lambda_{\alpha, i}=0} C_{\alpha, i} W^{\alpha} e_{i}+\epsilon\left(t-t_{0}\right) e^{-A\left(t-t_{0}\right)} \sum_{\Lambda_{\alpha, i}=0} C_{\alpha, i}\left(\sum_{j} \alpha_{j} \frac{W^{\alpha}}{W_{j}} \frac{d W_{j}}{d t_{0}}\right) e_{i} \\
+\epsilon A e^{-A\left(t-t_{0}\right)} \sum_{\Lambda_{\alpha, i} \neq 0} \frac{C_{\alpha, i}}{\Lambda_{\alpha, i}} e^{\left(t-t_{0}\right) \Lambda_{\alpha, i}} W^{\alpha} e_{i}-\epsilon e^{-A\left(t-t_{0}\right)} \sum_{\Lambda_{\alpha, i} \neq 0} \Lambda_{\alpha, i} \frac{C_{\alpha, i}}{\Lambda_{\alpha, i}} e^{\left(t-t_{0}\right) \Lambda_{\alpha, i}} W^{\alpha} e_{i} \\
+\epsilon e^{-A\left(t-t_{0}\right)} \sum_{\Lambda_{\alpha, i} \neq 0} \frac{C_{\alpha, i}}{\Lambda_{\alpha, i}} e^{\left(t-t_{0}\right) \Lambda_{\alpha, i}}\left(\sum_{j} \alpha_{j} \frac{W^{\alpha}}{W_{j}} \frac{d W_{j}}{d t_{0}}\right) e_{i}+\mathcal{O}\left(\epsilon^{2}\right)=\mathcal{O}\left(\epsilon^{2}\right) .
\end{gathered}
$$

It can be seen that

$$
A e^{-A\left(t-t_{0}\right)} W+e^{-A\left(t-t_{0}\right)} \frac{d W}{d t_{0}}=\mathcal{O}(\epsilon)
$$

and hence

$$
-A W=\frac{d W}{d t_{0}}+\mathcal{O}(\epsilon)
$$

Since $-A$ is diagonal, then $-\lambda_{j} W_{j}=\frac{d W_{j}}{d t_{0}}+\mathcal{O}(\epsilon)$. Therefore $\sum_{j} \alpha_{j} \frac{W^{\alpha}}{W_{j}} \frac{d W_{j}}{d t_{0}}=$ $W^{\alpha} \sum_{j} \alpha_{j} \lambda_{j}$. Also in the forth and sixth terms of (4.9), we can use the fact that $A e_{i}=\lambda_{i} e_{i}$. Hence (4.9) can be reduced to

$$
\begin{aligned}
A e^{-A\left(t-t_{0}\right)} W & +e^{-A\left(t-t_{0}\right)} \frac{d W}{d t_{0}}- \\
\epsilon e^{-A\left(t-t_{0}\right)} & \sum_{\Lambda_{\alpha, i}=0} C_{\alpha, i} W^{\alpha} e_{i}+\epsilon e^{-A\left(t-t_{0}\right)} \sum_{\Lambda_{\alpha, i}=0} C_{\alpha, i}\left(-\sum_{j} \alpha_{j} \lambda_{j}+\lambda_{i}\right) W^{\alpha} e_{i}+ \\
\epsilon e^{-A\left(t-t_{0}\right)} & \sum_{\Lambda_{\alpha, i} \neq 0} \frac{C_{\alpha, i}}{\Lambda_{\alpha, i}} e^{\left(t-t_{0}\right) \Lambda_{\alpha, i}}\left(-\sum_{j} \alpha_{j} \lambda_{j}-\Lambda_{\alpha, i}+\lambda_{i}\right) W^{\alpha} e_{i}=\mathcal{O}\left(\epsilon^{2}\right) .
\end{aligned}
$$


According to the definition of $\Lambda_{\alpha, i}$, the forth and fifth terms disappear. Hence

$$
\frac{d W}{d t_{0}}=-A W+\epsilon \sum_{\Lambda_{\alpha, i}=0} C_{\alpha, i} W^{\alpha} e_{i}+\mathcal{O}\left(\epsilon^{2}\right),
$$

where the initial value for this system, $W\left(T_{0}\right)$, can implicitly be found from

$$
w\left(T_{0}\right)=u_{0}=W\left(T_{0}\right)+\epsilon \sum_{\Lambda_{\alpha, i} \neq 0} \frac{C_{\alpha, i}}{\Lambda_{\alpha, i}} W\left(T_{0}\right)^{\alpha} e_{i}
$$

(4.10) is called the RG equation. By setting $t_{0}=t$, and substituting (4.10) back into (4.8) we find

$$
u(t)=W(t)+\epsilon \sum_{\Lambda_{\alpha, i} \neq 0} \frac{C_{\alpha, i}}{\Lambda_{\alpha, i}} W(t)^{\alpha} e_{i}+\mathcal{O}\left(\epsilon^{2}\right),
$$

which is the RG approximation to the system (4.1).

Therefore we have found an approximation to the system in 3 steps without introducing a new parameter.

Now let us recall the RG equation derived in chapter 2

$$
\begin{aligned}
\frac{d V}{d \sigma} & =\epsilon \sum_{\Lambda_{\alpha, i}=0} C_{\alpha, i} V(\sigma)^{\alpha} e_{i}+\mathcal{O}\left(\epsilon^{2}\right), \\
V\left(T_{0}\right) & =W_{0} .
\end{aligned}
$$

where

$$
W_{0}=e^{t_{0} A}\left(u_{0}-\epsilon \sum_{i=1}^{n}\left(\sum_{\Lambda_{\alpha, i} \neq 0} \frac{C_{\alpha, i}}{\Lambda_{\alpha, i}} u_{0}^{\alpha}\right) e_{i}\right) .
$$

The RG equations (4.12) and (4.10) are equivalent, indeed, $V(t)=e^{t A} W(t)$.

\subsection{Second Order RG Method}

In this section, we want to apply the second order RG method to three examples using the simplified method. 
Example 4.1. Let

$$
\ddot{y}+\epsilon \dot{y}+y=0, \quad y(0)=1, \dot{y}(0)=0, \epsilon \ll 1,
$$

be an initial value problem. In practice, the parameter $\epsilon$ is known but here it is an unknown parameter. Since this equation is a constant coefficient equation, the exact solution can be found:

$$
y(t) \sim e^{\frac{-\epsilon}{2} t}\left(d_{1} \sin \left(t-\frac{\epsilon^{2}}{8} t\right)+d_{2} \cos \left(t-\frac{\epsilon^{2}}{8} t\right)\right),
$$

for some constants $d_{1}, d_{2}$.

We are going to apply RG to this example. Let

$$
y(t)=y_{0}(t)+\epsilon y_{1}(t)+\epsilon^{2} y_{2}(t)+\mathcal{O}\left(\epsilon^{3}\right) .
$$

Thus

$$
\begin{aligned}
& y(t)=R \sin (t+\theta)-\frac{R}{2} \epsilon\left(\left(t-t_{0}\right) \sin (t+\theta)+\frac{1}{2} \cos (t+\theta)\right)+ \\
& \frac{R}{8} \epsilon^{2}\left(t-t_{0}\right)^{2} \sin (t+\theta)+\mathcal{O}\left(\epsilon^{3}\right) .
\end{aligned}
$$

We use the simplified RG method mentioned in the beginning of this chapter to find the amplitude equation. The RG condition is $\frac{d y}{d t_{0}}=\mathcal{O}\left(\epsilon^{3}\right)$. For ease of notation let $\frac{d R}{d t_{0}}=R^{\prime}$ and $\frac{d \theta}{d t_{0}}=\theta^{\prime}$.

$$
\begin{gathered}
\frac{\partial y}{\partial t_{0}}=R^{\prime} \sin (t+\theta)+R \theta^{\prime} \cos (t+\theta)-\frac{R^{\prime}}{2} \epsilon\left(\left(t-t_{0}\right) \sin (t+\theta)+\frac{1}{2} \cos (t+\theta)\right)- \\
\frac{R}{2} \epsilon\left(-\sin (t+\theta)+\left(t-t_{0}\right) \theta^{\prime} \cos (t+\theta)-\frac{\theta^{\prime}}{2} \sin (t+\theta)\right)+\frac{R^{\prime}}{8} \epsilon^{2}\left(t-t_{0}\right)^{2} \sin (t+\theta)+ \\
\frac{R^{\prime}}{8} \epsilon^{2}\left(-2\left(t-t_{0}\right) \sin (t+\theta)+\theta^{\prime}\left(t-t_{0}\right)^{2} \cos (t+\theta)\right)=\mathcal{O}\left(\epsilon^{3}\right) .
\end{gathered}
$$

Linear independence of sin and cos implies that $R^{\prime}=\mathcal{O}(\epsilon)$ and $\theta^{\prime}=\mathcal{O}(\epsilon)$. Hence by setting $t_{0}=t$ we find

$$
\begin{aligned}
R^{\prime} \sin (t+\theta)+R \theta^{\prime} \cos (t+\theta)-\frac{R^{\prime}}{2} \epsilon\left(\frac{1}{2} \cos (t+\theta)\right)- & \\
& \frac{R}{2} \epsilon\left(-\sin (t+\theta)-\frac{\theta^{\prime}}{2} \sin (t+\theta)\right)=\mathcal{O}\left(\epsilon^{3}\right) .
\end{aligned}
$$


This gives the following equations for the amplitude and phase:

$$
\begin{aligned}
R^{\prime}+\epsilon \frac{R}{2}-\frac{R \theta^{\prime}}{2} & =\mathcal{O}\left(\epsilon^{3}\right), \\
R \theta^{\prime}-\frac{\epsilon R^{\prime}}{4} & =\mathcal{O}\left(\epsilon^{3}\right) .
\end{aligned}
$$

Since $R^{\prime}=\mathcal{O}(\epsilon)$, by (4.14b) we find $R \theta^{\prime}=\mathcal{O}\left(\epsilon^{2}\right)$. If, for a moment, we want only the first order correction for $R,(4.14 \mathrm{a})$ gives

$$
R^{\prime}+\epsilon \frac{R}{2}=\mathcal{O}\left(\epsilon^{2}\right)
$$

Hence

$$
R(t)=R(0) e^{-\frac{\epsilon}{2} t}+\mathcal{O}\left(\epsilon^{2} t\right)
$$

Inserting (4.15) and its derivative into (4.14b) imply

$$
\theta^{\prime}=-\frac{\epsilon^{2}}{8}
$$

To find higher order corrections for $R$, we reinsert (4.16) into (4.14a). Thus

$$
\begin{aligned}
R(t) & =R(0) e^{-\left(\frac{\epsilon}{2}+\frac{\epsilon^{2}}{16}\right) t}+\mathcal{O}\left(\epsilon^{3} t\right), \\
\theta(t) & =-\frac{\epsilon^{2}}{8} t+\theta(0)+\mathcal{O}\left(\epsilon^{3} t\right) .
\end{aligned}
$$

Hence the final solution reads

$$
y(t)=R(0) e^{-\left(\frac{\epsilon}{2}+\frac{\epsilon^{2}}{16}\right) t}\left(\sin \left(t-\frac{\epsilon^{2}}{8} t+\theta(0)\right)-\frac{\epsilon}{4} \cos \left(t-\frac{\epsilon^{2}}{8} t+\theta(0)\right)\right)+\mathcal{O}\left(\epsilon^{3} t\right) .
$$

This is the outcome of the second order RG method, while

$$
y(t)=R(0) e^{-\frac{\epsilon}{2} t}\left(\sin (t+\theta(0))-\frac{\epsilon}{4} \cos (t+\theta(0))\right)
$$

is the first order RG. See the figures (4.1), (4.2), (4.3) and (4.4) to see how first and second order RG approximations estimate solutions to (4.13). 


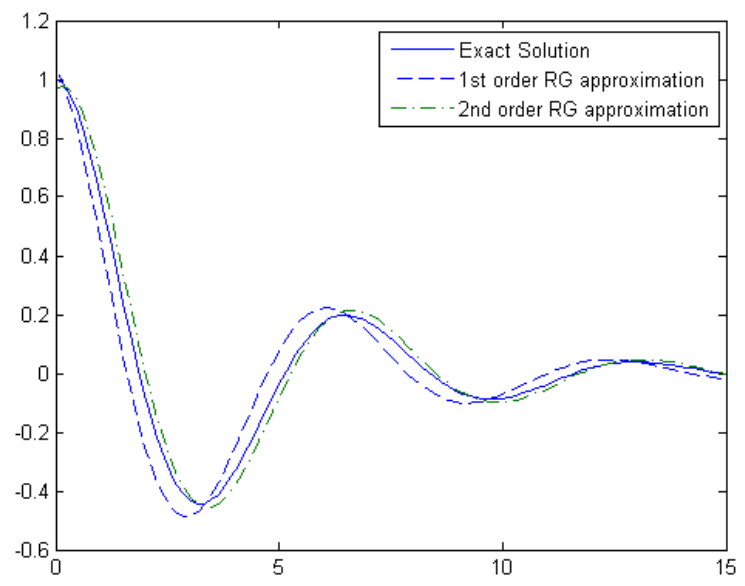

Figure 4.1: This figure shows how precise the first and second order RG outcomes approximate the solutions to the initial value problem (4.13) for small values of $t$. For all these figures, $\epsilon=.5$.

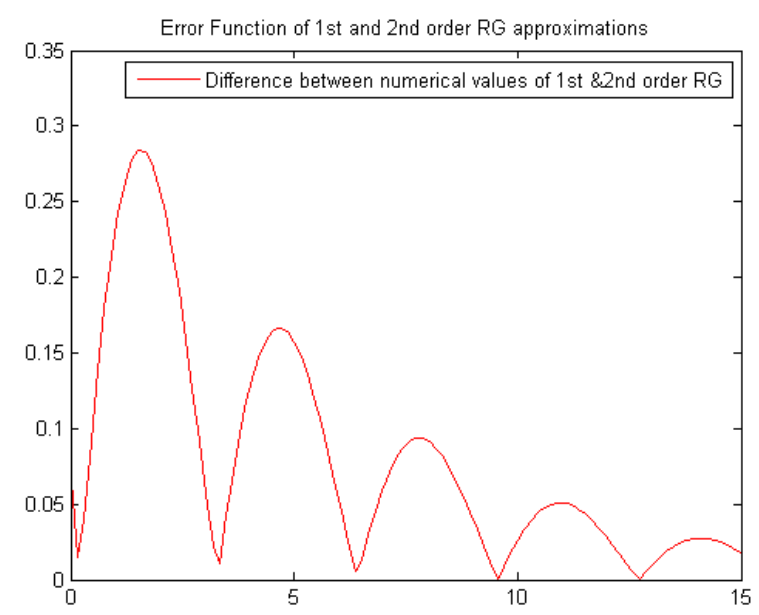

Figure 4.2: This figure shows the difference between the 1st and 2nd order $\mathrm{RG}$ outcomes from small to large values of $t$. This illustrates that the difference between the 1st and 2nd order RG grows in $t \cos t$ up to a maximum and then converges to zero. 


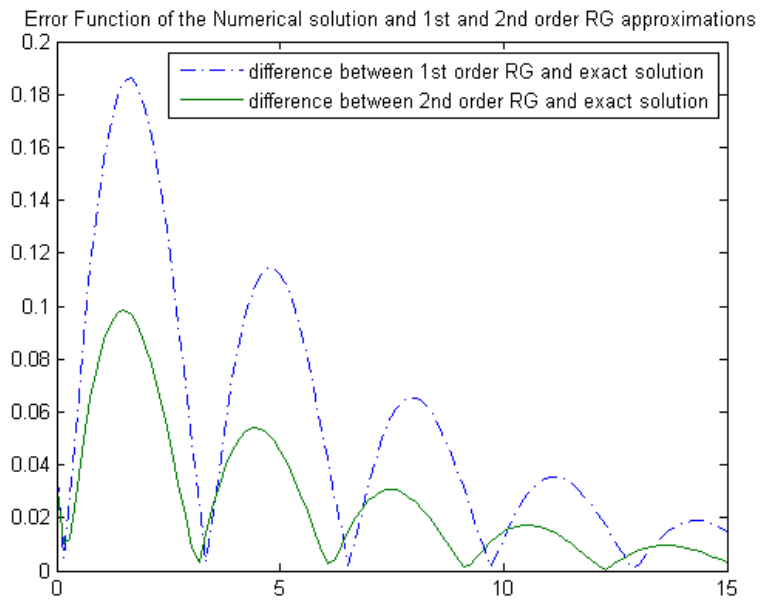

Figure 4.3: This figure shows the difference between the 1st order RG and the exact solution as well as 2nd order RG and the exact solution for small values of $t$.

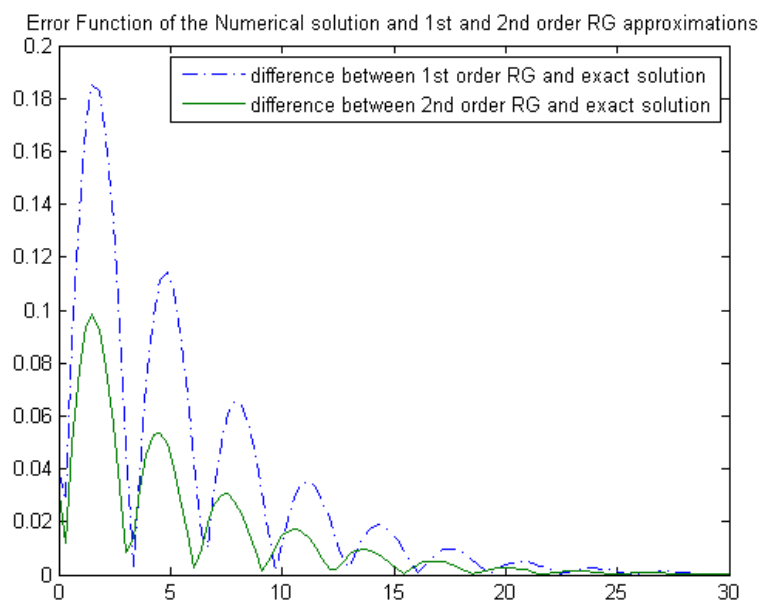

Figure 4.4: This figure shows the difference between the 1st order RG and the exact solution as well as 2nd order RG and the exact solution for large values of $t$. The error becomes maximum around $t=3$ and then it declines. 
Example 4.2. In this example we want to find the second order RG equation for the Rayleigh oscillator,

$$
\ddot{y}+y=\epsilon\left(\dot{y}-\frac{1}{3}(\dot{y})^{3}\right) .
$$

By assuming a naive expansion representation for the solution up to and including $\mathcal{O}\left(\epsilon^{2}\right)$, we find

$$
\begin{aligned}
& \ddot{y}_{0}+y_{0}=0, \\
& \ddot{y}_{1}+y_{1}=\dot{y}_{0}-\frac{1}{3}\left(\dot{y}_{0}\right)^{3}, \\
& \ddot{y}_{2}+y_{2}=\dot{y}_{1}\left(1-\left(\dot{y}_{0}\right)^{2}\right) .
\end{aligned}
$$

Doing straightforward calculations give the solution to (4.17a) and (4.17b)

$$
\begin{aligned}
& y_{0}(t)=A e^{i\left(t-t_{0}\right)}+c . c, \\
& y_{1}(t)=\frac{i}{24} A^{3} e^{i\left(t-t_{0}\right)}+\frac{1}{2} A\left(1-A A^{*}\right)\left(t-t_{0}\right) e^{i\left(t-t_{0}\right)}-\frac{i}{24} A^{3} e^{3 i\left(t-t_{0}\right)}+\text { c.c. }
\end{aligned}
$$

If we have the initial condition $y(0)=a$ such that $a$ is independent of $\epsilon$, we should have $y_{0}(0)=a$ and $y_{i}(0)=0$ for all $i>0$. This is why we have chosen the integral constant of $y_{1}$ so that $y_{1}(0)=0$. We will impose such condition on $y_{2}$ as well.

The nonhomogeneous part is :

$$
\begin{array}{r}
\dot{y}_{1}\left(1-\left(\dot{y}_{0}\right)^{2}\right)=\left(\left(-\frac{A^{3}}{24}+\frac{1}{2} A\left(1-A A^{*}\right)\right) e^{i\left(t-t_{0}\right)}+\frac{i}{2} A\left(1-A A^{*}\right)\left(t-t_{0}\right) e^{i\left(t-t_{0}\right)}+\right. \\
\frac{A^{3}}{8} e^{3 i\left(t-t_{0}\right)}+\left(\frac{-A^{* 3}}{24}+\frac{1}{2} A^{*}\left(1-A A^{*}\right)\right) e^{-i\left(t-t_{0}\right)}-\frac{i}{2} A^{*}\left(1-A A^{*}\right)\left(t-t_{0}\right) e^{-i\left(t-t_{0}\right)}+ \\
\left.\frac{A^{* 3}}{8} e^{-3 i\left(t-t_{0}\right)}\right)\left(1-2 A A^{*}+A^{2} e^{2 i\left(t-t_{0}\right)}+A^{* 2} e^{-2 i\left(t-t_{0}\right)}\right),
\end{array}
$$

or

$\dot{y}_{1}\left(1-\left(\dot{y}_{0}\right)^{2}\right)=D_{1} e^{i\left(t-t_{0}\right)}+D_{2}\left(t-t_{0}\right) e^{i\left(t-t_{0}\right)}+D_{3} e^{3 i\left(t-t_{0}\right)}+D_{4}\left(t-t_{0}\right) e^{3 i\left(t-t_{0}\right)}+D_{5} e^{5 i\left(t-t_{0}\right)}$, 
where

$$
\begin{aligned}
& D_{1}=\left(-\frac{A^{3}}{24}+\frac{1}{2} A\left(1-A A^{*}\right)\right)\left(1-2 A A^{*}\right)+\frac{1}{8} A^{3} A^{* 2}+A^{2}\left(\frac{-A^{* 3}}{24}+\frac{1}{2} A^{*}\left(1-A A^{*}\right)\right), \\
& D_{2}=\left(\frac{i}{2} A\left(1-A A^{*}\right)\right)\left(1-2 A A^{*}\right)+A^{2}\left(\frac{i}{2} A^{*}\left(1-A A^{*}\right)\right), \\
& D_{3}=A^{2}\left(-\frac{A^{3}}{24}+\frac{1}{2} A\left(1-A A^{*}\right)\right)+\frac{1}{8} A^{3}\left(1-2 A A^{*}\right), \\
& D_{4}=A^{2}\left(\frac{i}{2} A\left(1-A A^{*}\right)\right), \\
& D_{5}=\frac{1}{8} A^{5} .
\end{aligned}
$$

We simplify $D_{i}$ s to get

$$
\begin{aligned}
& D_{1}=-\frac{A^{3}}{24}+\frac{1}{2} A^{4} A^{*}-\frac{1}{24} A^{2} A^{* 3}+\frac{1}{2} A+\frac{5}{8} A^{3} A^{* 2}-A^{2} A^{*}, \\
& D_{2}=\frac{i}{2}\left(A-4 A^{2} A^{*}+3 A^{3} A^{* 2}\right), \\
& D_{3}=-\frac{1}{24} A^{5}+\frac{5}{8} A^{3}-\frac{1}{24} A^{4} A^{*}, \\
& D_{4}=\frac{i}{2}\left(A^{3}-A^{4} A^{*}\right), \\
& D_{5}=\frac{1}{8} A^{5} .
\end{aligned}
$$

Using the variation of parameters method we find

$$
\begin{gathered}
y_{2}(t)=\frac{-i}{2}\left(A_{1} e^{i\left(t-t_{0}\right)}+H_{1}\left(t-t_{0}\right) e^{i\left(t-t_{0}\right)}+H_{2}\left(t-t_{0}\right)^{2} e^{i\left(t-t_{0}\right)}+\right. \\
\left.H_{3} e^{3 i\left(t-t_{0}\right)}+H_{4}\left(t-t_{0}\right) e^{3 i\left(t-t_{0}\right)}+H_{5} e^{5 i\left(t-t_{0}\right)}\right)+ \text { c.c. }
\end{gathered}
$$

We can choose $A_{1}$ such that $y_{2}\left(t_{0}\right)=0$. Hence $A_{1}=-H_{3}-H_{5}$. Now, using (4.18), $H_{i}$ read

$$
\begin{aligned}
& H_{1}=D_{1}+\frac{i}{2} D_{2}=\frac{A}{4}\left(1-\frac{A^{2} A^{* 2}}{2} \frac{A^{2}}{6}-\frac{A A^{* 3}}{6}+\frac{A^{3} A^{*}}{3}\right) \\
& H_{2}=\frac{D_{2}}{2}=\frac{i}{4} A\left(1-4 A A^{*}+3 A^{2} A^{* 2}\right) \\
& H_{3}=\frac{-i}{4} D_{3}+\frac{3}{16} D_{4}=\frac{-i}{32} A^{3}\left(2-\frac{A^{2}}{3}-3 A A^{*}\right) \\
& H_{4}=-\frac{i}{4} D_{4}=\frac{-1}{8} A^{3}\left(1-A A^{*}\right) \\
& H_{5}=-\frac{i}{12} D_{5}=\frac{i}{96} A^{5}
\end{aligned}
$$


In summary, we have

$$
\begin{array}{r}
y(t)=A e^{i\left(t-t_{0}\right)}+c . c+\epsilon\left(\frac{i}{24} A^{3} e^{i\left(t-t_{0}\right)}+\frac{1}{2} A(1-A * A)\left(t-t_{0}\right) e^{i\left(t-t_{0}\right)}-\right. \\
\left.\frac{i}{24} A^{3} e^{3 i\left(t-t_{0}\right)}+c . c\right)+\epsilon^{2} y_{2}(t) .
\end{array}
$$

Now we want to absorb integral constants of $y_{1}$ and $y_{2}$. Let

$$
A=a_{0}+\epsilon a_{1}+\epsilon^{2} a_{2}+\mathcal{O}\left(\epsilon^{3}\right)
$$

To absorb coefficients of $e^{i\left(t-t_{0}\right)}$ up to second order, we must have:

$$
\begin{aligned}
& a_{0}=B \\
& a_{1}=\frac{-i}{24} B^{3} \\
& a_{2}=-H_{3}-H_{5}=-\frac{B^{3}}{32}\left(1-\frac{3}{2} B B^{*}-\frac{B^{2}}{6}\right) .
\end{aligned}
$$

Then

$$
A=B-\epsilon \frac{i}{24} B^{3}-\epsilon^{2} \frac{B^{3}}{32}\left(1-\frac{3}{2} B B^{*}-\frac{B^{2}}{6}\right)+\mathcal{O}\left(\epsilon^{3}\right) .
$$

Now we should substitute this into (4.19) to find the coefficients with respect to $B$. Note plugging (4.20) in to $y_{0}$ eliminates integral constants of $y_{1}$ and $y_{2}$ and will not have any contribution in canceling other coefficients of $y_{2}$; because they are of $\mathcal{O}\left(\epsilon^{3}\right)$ or higher. For ease of notation, let $b=\frac{-B^{3}}{24}$. Hence

$$
\begin{gathered}
y(t)=B e^{i\left(t-t_{0}\right)}+\epsilon\left(\frac{1}{2}\left(B+\epsilon i b+\mathcal{O}\left(\epsilon^{2}\right)\right)\left(1-\left(B+\epsilon i b+\mathcal{O}\left(\epsilon^{2}\right)\right)\left(B+\epsilon i b+\mathcal{O}\left(\epsilon^{2}\right)\right)^{*}\right) \times\right. \\
\left.\left(t-t_{0}\right) e^{i\left(t-t_{0}\right)}-\frac{i}{24}\left(B+\epsilon i b+\mathcal{O}\left(\epsilon^{2}\right)\right)^{3} e^{3 i\left(t-t_{0}\right)}\right)+\epsilon^{2} y_{2 B}(t)+c . c+\mathcal{O}\left(\epsilon^{3}\right), \quad
\end{gathered}
$$

where $y_{2}(t)$ is the same as $y_{2}(t)$ except for $A$ which is replaced by $B$ and the homogeneous solution is cancelled out. By expanding the quadratic and cubic terms in (4.21), we obtain

$$
\begin{aligned}
y(t)=B e^{i\left(t-t_{0}\right)}+\epsilon\left(\frac{1}{2}(B+\right. & \epsilon i b) \\
& \left(1-B B^{*}-\epsilon\left(i b B^{*}-i B b^{*}\right)\right)\left(t-t_{0}\right) e^{i\left(t-t_{0}\right)} \\
& \left.-\frac{i}{24}\left(B^{3}+3 i b B^{3} \epsilon\right) e^{3 i\left(t-t_{0}\right)}\right)+\epsilon^{2} y_{2 B}(t)+c . c+\mathcal{O}\left(\epsilon^{3}\right) .
\end{aligned}
$$


Thus

$$
\begin{gathered}
y(t)=B e^{i\left(t-t_{0}\right)}+\epsilon \frac{1}{2} B\left(1-B B^{*}\right)\left(t-t_{0}\right) e^{i\left(t-t_{0}\right)}+ \\
\epsilon^{2}\left(-B\left(i b B^{*}-i B b^{*}\right)+i b\left(1-B B^{*}\right)\right)-\epsilon \frac{i}{24} B^{3} e^{3 i\left(t-t_{0}\right)}- \\
\epsilon^{2} \frac{1}{8 \times 24} B^{5} e^{3 i\left(t-t_{0}\right)}+\epsilon^{2} y_{2}(t)+c . c+\mathcal{O}\left(\epsilon^{3}\right) .
\end{gathered}
$$

Terms of $\mathcal{O}\left(\epsilon^{2}\right)$ in (4.22) cancel some of terms in $H_{1}$ and add one term:

$$
\begin{gathered}
y(t)=B e^{i\left(t-t_{0}\right)}+\epsilon\left(\frac{1}{2} B\left(1-B B^{*}\right)\left(t-t_{0}\right) e^{i\left(t-t_{0}\right)}-\frac{i}{24} B^{3} e^{3 i\left(t-t_{0}\right)}\right)+\epsilon^{2}( \\
-\frac{i B}{8}\left(1-\frac{B^{2} B^{* 2}}{2}\right)\left(t-t_{0}\right) e^{i\left(t-t_{0}\right)}+\frac{B}{8}\left(1-4 B B^{*}+3 B^{2} B^{* 2}\right)\left(t-t_{0}\right)^{2} e^{i\left(t-t_{0}\right)}- \\
\frac{B^{3}}{64}\left(2-\frac{B^{2}}{3}-3 B B^{*}\right) e^{3 i\left(t-t_{0}\right)}-\frac{i B^{3}}{16}\left(1-B B^{*}\right)\left(t-t_{0}\right) e^{3 i\left(t-t_{0}\right)}- \\
\left.\frac{1}{192} B^{5} e^{5 i\left(t-t_{0}\right)}-\frac{1}{192} B^{5} e^{3 i\left(t-t_{0}\right)}\right)+c \cdot c+\mathcal{O}\left(\epsilon^{3}\right) .
\end{gathered}
$$

We now use RG condition, $\left.\frac{\partial y}{\partial t_{0}}\right|_{t_{0}=t}=\mathcal{O}\left(\epsilon^{3}\right)$, to find the RG equation. The first observation is

$$
\frac{\partial y}{\partial t_{0}}=B^{\prime} e^{i\left(t-t_{0}\right)}-i B e^{i\left(t-t_{0}\right)}+B^{* \prime} e^{-i\left(t-t_{0}\right)}+i B^{*} e^{-i\left(t-t_{0}\right)}+\mathcal{O}(\epsilon)=0
$$

The linear independence of $e^{i\left(t-t_{0}\right)}$ and $e^{-i\left(t-t_{0}\right)}$ implies

$$
\begin{aligned}
\left.B^{\prime}\right|_{t_{0}=t} & =i B(t)+\mathcal{O}(\epsilon), \\
\left.B^{* \prime}\right|_{t_{0}=t} & =-i B^{*}(t)+\mathcal{O}(\epsilon) .
\end{aligned}
$$

Having these in mind, we try to find the RG equation:

$$
\begin{aligned}
&\left.\frac{\partial y}{\partial t_{0}}\right|_{t_{0}=t}=B^{\prime}- i B+\epsilon\left(-\frac{1}{2} B\left(1-B B^{*}\right)-\frac{i}{8} B^{\prime} B^{2}-\frac{B^{3}}{8}\right)+ \\
& \epsilon^{2}\left(\frac{i B}{8}\left(1-\frac{B^{2} B^{* 2}}{2}\right)+K+\frac{3 i}{32}\left(1-\frac{B^{2}}{6}-\frac{3}{2} B B^{*}\right)+\right. \\
&\left.\frac{i B^{3}}{16}\left(1-B B^{*}\right)+\frac{8 i}{192} B^{5}-\frac{10}{192} B^{\prime} B^{4}\right)=\mathcal{O}\left(\epsilon^{3}\right),
\end{aligned}
$$


where ${ }^{\prime}$ is derivative with respect to $t_{0}$ and

$$
\begin{array}{r}
K=-\left(\frac{B^{3}}{32}\left(1-\frac{B^{2}}{6}-\frac{3}{2} B B^{*}\right)\right)^{\prime}=-\frac{3}{32} B^{2} B^{\prime}\left(1-\frac{B^{2}}{6}-\frac{3}{2} B B^{*}\right)-\frac{1}{32} B^{3}\left(-\frac{B^{\prime} B}{3}-\right. \\
\left.\frac{3}{2} B^{\prime} B^{*}-\frac{3}{2} B B^{* \prime}\right)=-\frac{3}{32} B^{2} B^{\prime}\left(1-\frac{B^{2}}{6}-\frac{3}{2} B B^{*}\right)+\frac{1}{96} B^{4} B^{\prime} .
\end{array}
$$

We expand the derivative terms and substitute (4.24)s into them:

$$
\begin{aligned}
& B^{\prime}-i B+\epsilon(-\left.\frac{1}{2} B\left(1-B B^{*}\right)-\frac{i}{8} B^{2}(i B+\mathcal{O}(\epsilon))-\frac{B^{3}}{8}\right)+ \\
& \epsilon^{2}\left(\frac{i B}{8}\left(1-\frac{B^{2} B^{* 2}}{2}\right)+K+\frac{3 i}{32} B^{3}\left(1-\frac{B^{2}}{6}-\frac{3}{2} B B^{*}\right)+\right. \\
&\left.\frac{i}{16} B^{3}\left(1-B B^{*}\right)+\frac{8 i}{192} B^{5}-\frac{10}{192} B^{4}(i B)\right)=\mathcal{O}\left(\epsilon^{3}\right),
\end{aligned}
$$

where

$$
K=-\frac{3}{32} B^{2}(i B)\left(1-\frac{B^{2}}{6}-\frac{3}{2} B B^{*}\right)+\frac{1}{96} B^{4}(i B)=-\frac{3 i}{32} B^{3}\left(1-\frac{B^{2}}{6}-\frac{3}{2} B B^{*}\right)+\frac{i}{96} B^{5} .
$$

(4.25) implies

$$
\begin{gathered}
B^{\prime}-i B-\frac{\epsilon}{2} B\left(1-B B^{*}\right)+\frac{\epsilon}{8} B^{3}-\frac{i}{8} B^{2}(\mathcal{O}(\epsilon))-\frac{\epsilon}{8} B^{3}+ \\
\epsilon^{2}\left(\frac{i B}{8}\left(1-\frac{B^{2} B^{* 2}}{2}\right)+K+\frac{3 i}{32} B^{3}\left(1-\frac{B^{2}}{6}-\frac{3}{2} B B^{*}\right)+\right. \\
\left.\frac{i}{16} B^{3}\left(1-B B^{*}\right)+\frac{8 i}{192} B^{5}-\frac{10 i}{192} B^{5}\right)=\mathcal{O}\left(\epsilon^{3}\right) .
\end{gathered}
$$

Note that $\mathcal{O}(\epsilon)=\epsilon\left(\frac{1}{2} B\left(1-B B^{*}\right)+\frac{i}{8} B^{\prime} B^{2}+\frac{B^{3}}{8}\right)+\mathcal{O}\left(\epsilon^{2}\right)$. We substitute this into (4.26) and do some calculations to get

$$
\begin{aligned}
& B^{\prime}-i B-\frac{\epsilon}{2} B\left(1-B B^{*}\right)-\frac{i \epsilon^{2}}{16} B^{3}\left(1-B B^{*}\right)+\frac{\epsilon^{2}}{64} B^{4} B^{\prime}-\frac{i \epsilon^{2}}{64} B^{5}+ \\
& \epsilon^{2}\left(\frac{i B}{8}\left(1-\frac{B^{2} B^{* 2}}{2}\right)+K+\frac{3 i}{32} B^{3}\left(1-\frac{B^{2}}{6}-\frac{3}{2} B B^{*}\right)+\right. \\
&\left.\frac{i}{16} B^{3}\left(1-B B^{*}\right)-\frac{i}{96} B^{5}\right)=\mathcal{O}\left(\epsilon^{3}\right) .
\end{aligned}
$$


Again by using (4.24a) we obtain

$$
\begin{gathered}
B^{\prime}-i B-\frac{\epsilon}{2} B\left(1-B B^{*}\right)-\frac{i \epsilon^{2}}{16} B^{3}\left(1-B B^{*}\right)+\frac{i \epsilon^{2}}{64} B^{5}-\frac{i \epsilon^{2}}{64} B^{5}+ \\
\epsilon^{2}\left(\frac{i B}{8}\left(1-\frac{B^{2} B^{* 2}}{2}\right)+\frac{3 i}{32} B^{3}\left(1-\frac{B^{2}}{6}-\frac{3}{2} B B^{*}\right)+\frac{i}{96} B^{5}-\frac{3 i}{32} B^{3}\left(1-\frac{B^{2}}{6}-\frac{3}{2} B B^{*}\right)+\right. \\
\left.\frac{i}{16} B^{3}\left(1-B B^{*}\right)-\frac{i}{96} B^{5}\right)=\mathcal{O}\left(\epsilon^{3}\right) .
\end{gathered}
$$

Now we observe that several terms in (4.27) cancel each other. Hence

$$
B^{\prime}=i B+\frac{\epsilon}{2} B\left(1-B B^{*}\right)-\frac{i \epsilon^{2}}{8} B\left(1-\frac{B^{2} B^{* 2}}{2}\right)+\mathcal{O}\left(\epsilon^{3}\right)
$$

To solve this equation, we use polar coordinates. Let $B=\frac{R}{2} e^{i \theta}$. Thus

$$
\frac{R^{\prime}}{2} e^{i \theta}+\frac{i R \theta^{\prime}}{2} e^{i \theta}=i \frac{R}{2} e^{i \theta}+\frac{\epsilon}{2} \frac{R}{2} e^{i \theta}\left(1-\frac{R^{2}}{4}\right)-i \frac{\epsilon^{2}}{8} \frac{R}{2} e^{i \theta}\left(1-\frac{R^{4}}{32}\right)+\mathcal{O}\left(\epsilon^{3}\right) .
$$

Therefore the amplitude and the phase equations read

$$
\begin{aligned}
R^{\prime} & =\frac{\epsilon}{2} R\left(1-\frac{R^{2}}{4}\right)+\mathcal{O}\left(\epsilon^{3}\right), \\
\theta^{\prime} & =1-\frac{\epsilon^{2}}{8}\left(1-\frac{R^{4}}{32}\right)+\mathcal{O}\left(\epsilon^{3}\right) .
\end{aligned}
$$

Solving (4.29) turns out

$$
\begin{aligned}
R(t) & =\frac{R(0)}{\sqrt{e^{-\epsilon t}+\frac{1}{4} R(0)^{2}\left(1-e^{-\epsilon t}\right)}}+\mathcal{O}\left(\epsilon^{3}\right), \\
\theta(t) & =c+\left(1-\frac{\epsilon^{2}}{16}\right) t-\frac{\epsilon}{16} \ln \frac{1}{4}\left(\left(4-R^{2}(0)\right) e^{-\epsilon t}+R^{2}(0)\right)- \\
\frac{3 \epsilon}{16} & \frac{R^{2}(0)}{\left(4-R^{2}(0)\right) e^{-\epsilon t}+R^{2}(0)}+\mathcal{O}\left(\epsilon^{3}\right),
\end{aligned}
$$

where $c$ is an integral constant. The values $R(0)$ and $c$ can be determined by using initial conditions.

Remark 4.3. The results of the second order RG applied to the Rayleigh Oscillator, (4.29), shows that the amplitude equation achieved in (2.19) was up to $\mathcal{O}\left(\epsilon^{3}\right)$, since there is no more correction in the second order. 


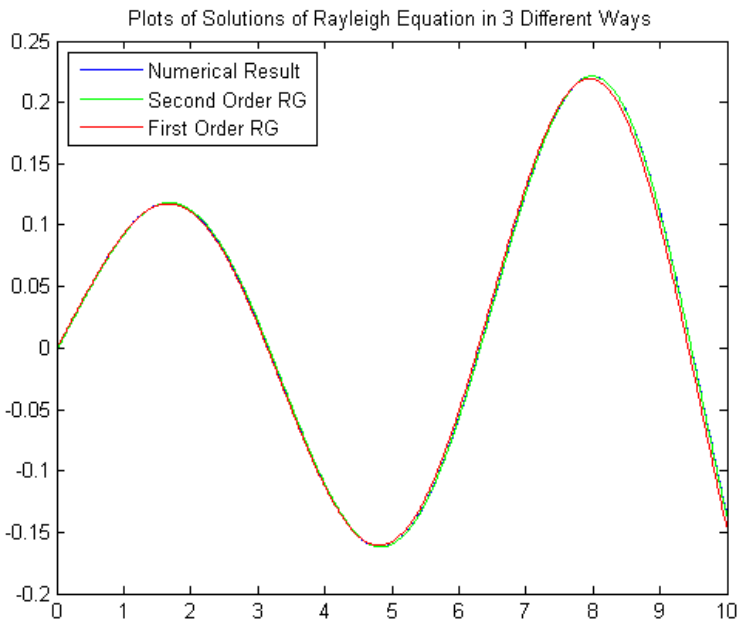

Figure 4.5: This figure shows plots of solutions to the Rayleigh equation using numerical methods and the 1st and 2nd order RG method $(\epsilon=.2$ and $a=.05)$. Notice that the numerical solution figure (the blue one) is plotted by using the MATLAB command ode45 (This command applies the Runge-Kutta method of order 4 or 5 to solve nonlinear initial value problems).

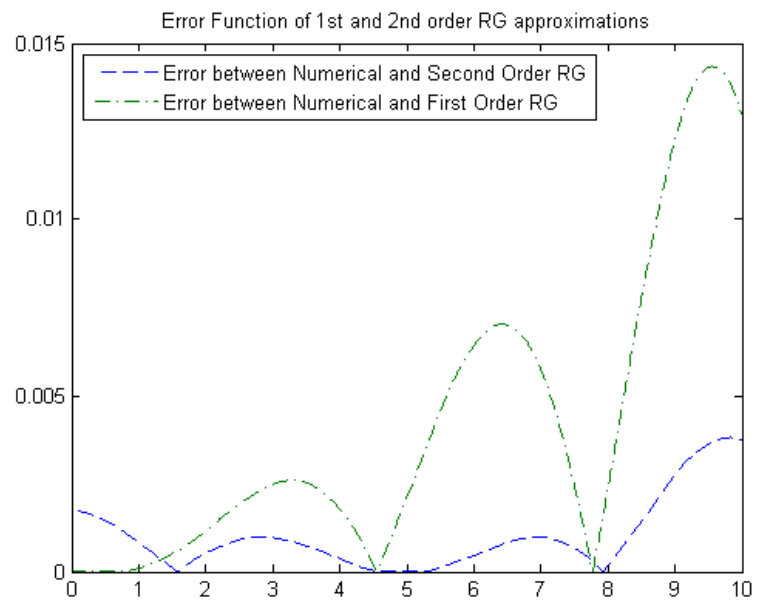

Figure 4.6: This figure shows the difference between the 1st order RG applied to Rayleigh's equation and its exact solution as well as the 2nd order RG applied to Rayleigh's equation and its exact solution for small values of $t$. 
Remark 4.4. In (4.23), the terms $\epsilon t e^{i t}, \epsilon^{2} t e^{i t}$ and $\epsilon^{2} t e^{i t}$ are called resonant secular terms, the term $\epsilon^{2} t e^{3 i t}$ is called non-resonant secular term and the terms $\epsilon e^{i t}, \epsilon^{2} e^{i t}$, $\epsilon e^{3 i t}, \epsilon^{2} e^{3 i t}$ and $\epsilon^{2} e^{5 i t}$ are called nonresonant terms. According to the new research, done by Oono and Shiwa [19], we only should care about the resonant secular terms to find the RG equation. Hence we have only to apply the RG condition to

$$
\begin{gathered}
y(t)=B e^{i\left(t-t_{0}\right)}+\epsilon\left(\frac{1}{2} B\left(1-B B^{*}\right)\left(t-t_{0}\right) e^{i\left(t-t_{0}\right)}\right)+\epsilon^{2}\left(-\frac{i B}{8}\left(1-\frac{B^{2} B^{* 2}}{2}\right) \times\right. \\
\left.\left(t-t_{0}\right) e^{i\left(t-t_{0}\right)}+\frac{B}{8}\left(1-4 B B^{*}+3 B^{2} B^{* 2}\right)\left(t-t_{0}\right)^{2} e^{i\left(t-t_{0}\right)}\right)+c . c+\mathcal{O}\left(\epsilon^{3}\right) .
\end{gathered}
$$

Applying the RG condition to (4.30) immediately reproduces the RG equation in (4.28).

To end this chapter, we consider the nonlinear Carrier equation. Recall that the result of applying the first order RG method to this differential equation reproduces the exact solution up to a certain correction. In the following example, we apply the second order RG method to reinforce the idea that the second order RG vanishes:

Example 4.5. Recall the Carrier equation

$$
(x+\epsilon y) y^{\prime}+y=1, \quad y(1)=2, \quad 0 \leq x \leq 1
$$

In chapter 1 , we observed that, by a natural change of variable, the Carrier equation changes to

$$
(x+Y) Y^{\prime}+Y=\epsilon, \quad Y(1)=2 \epsilon .
$$

where $Y=\epsilon y$. Here the aim is to investigate the second order RG.

Let

$$
Y(x)=Y_{0}(x)+\epsilon Y_{1}(x)+\epsilon^{2} Y_{2}(x)+\mathcal{O}\left(\epsilon^{3}\right) .
$$


By substituting the naive expansion (4.31) into the equation (4.31) we get

$$
\begin{aligned}
& Y_{0}=-x+\sqrt{A_{0}+x^{2}}, \\
& Y_{1}=\frac{A_{1}}{\sqrt{A_{0}+x^{2}}}+\frac{x-x_{0}}{\sqrt{A_{0}+x^{2}}}, \\
& Y_{2}=\frac{A_{2}}{\sqrt{A_{0}+x^{2}}}-\frac{A_{1}^{2}}{2 \sqrt{\left(A_{0}+x^{2}\right)^{3}}}-\frac{A_{1}\left(x-x_{0}\right)}{\sqrt{\left(A_{0}+x^{2}\right)^{3}}}-\frac{\left(x-x_{0}\right)^{2}}{2 \sqrt{\left(A_{0}+x^{2}\right)^{3}}}
\end{aligned}
$$

for some constants $A_{1}$ and $A_{2}$. Thus

$$
\begin{aligned}
& Y(x)=-x+\sqrt{A_{0}+x^{2}}+\epsilon\left(\frac{A_{1}}{\sqrt{A_{0}+x^{2}}}+\frac{x-x_{0}}{\sqrt{A_{0}+x^{2}}}\right)- \\
& \epsilon^{2}\left(\frac{-A_{2}}{\sqrt{A_{0}+x^{2}}}+\frac{A_{1}^{2}}{2 \sqrt{\left(A_{0}+x^{2}\right)^{3}}}+\frac{A_{1}\left(x-x_{0}\right)}{\sqrt{\left(A_{0}+x^{2}\right)^{3}}}+\frac{\left(x-x_{0}\right)^{2}}{2 \sqrt{\left(A_{0}+x^{2}\right)^{3}}}\right)+\mathcal{O}\left(\epsilon^{3}\right) .
\end{aligned}
$$

By substituting the change of variable $A_{0}=A\left(x_{0}\right)\left(1-2 A_{1} \epsilon-2 A_{2} \epsilon^{2}\right)+\mathcal{O}\left(\epsilon^{3}\right)$ into (4.32) we find

$$
Y(x)=-x+\sqrt{A+x^{2}}+\epsilon \frac{x-x_{0}}{\sqrt{A+x^{2}}}-\frac{1}{2} \epsilon^{2}\left(\frac{A_{1}^{2}}{\sqrt{\left(A+x^{2}\right)^{3}}}+\frac{\left(x-x_{0}\right)^{2}}{\sqrt{\left(A+x^{2}\right)^{3}}}\right)+\mathcal{O}\left(\epsilon^{3}\right)
$$

where $A=A\left(x_{0}\right)$.

By differentiating with respect to $x_{0}$ we get

$$
\begin{array}{r}
\frac{\partial Y}{\partial x_{0}}=\frac{A^{\prime}}{2 \sqrt{A+x^{2}}}+\epsilon \frac{-1}{\sqrt{A+x^{2}}}-\frac{1}{2} \epsilon A^{\prime} \frac{x-x_{0}}{\sqrt{\left(A+x^{2}\right)^{3}}}+\frac{1}{4} \epsilon^{2} A^{\prime} \frac{A_{1}^{2}}{\sqrt{\left(A+x^{2}\right)^{3}}}- \\
\epsilon^{2} \frac{x-x_{0}}{\sqrt{\left(A+x^{2}\right)^{3}}}-\frac{3}{4} \epsilon^{2} A^{\prime} \frac{\left(x-x_{0}\right)^{2}}{\sqrt{\left(A+x^{2}\right)^{5}}}+\mathcal{O}\left(\epsilon^{3}\right),
\end{array}
$$

where $A^{\prime}=\frac{d A}{d x_{0}}$.

Now by applying the RG condition $\left.\frac{\partial Y}{\partial x_{0}}\right|_{x_{0}=x}=\mathcal{O}\left(\epsilon^{3}\right)$ we find $A^{\prime}=\mathcal{O}(\epsilon)$. Hence

$$
A^{\prime}(x)=2 \epsilon x+\mathcal{O}\left(\epsilon^{3}\right)
$$

Now compare this to the result of the first order RG, (2.100). 


\section{Chapter 5}

\section{The Relationship Between the Renormal- ization Group Method And the Normal FORM THEORY}

In this chapter we will study the first order Normal Form theory (see [25]) and its relationship with the first order RG method (see [16]). In addition, at the end of this chapter a geometric interpretation for the RG method as well as the normal form theory will be presented which clarifies the relationship between RG and normal form (see [16].)

\subsection{The Normal Form Theory}

Consider the autonomous system of differential equations

$$
\left\{\begin{array}{l}
\dot{u}+A u=\sum_{\alpha, i} C_{\alpha, i} u^{\alpha} e_{i}, \\
u\left(t_{0}\right)=u_{0},
\end{array}\right.
$$

is given. We assume that the origin is an equilibrium point for the system. Then the aim is to investigate the solution of the system in the neighborhood of the origin. In the (classical) Normal Form (NF) theory, we use a near identity change of variables, $u=y+h(y)$ such that $h(y)=\mathcal{O}\left(|y|^{2}\right)$, to remove some nonlinear terms to simplify the system. The classical normal forms are not the simplest normal forms and their further simplification is possible via additional near-identity transformations. 
Therefore, one may use such additional near identity transformations to eliminate or modify certain terms. This causes the non-uniqueness. This may be avoided by setting a rule to uniquely choose the near identity transformations in the classical normal forms[22]. ${ }^{1}$

Now consider the system

$$
\left\{\begin{array}{l}
\dot{u}+A u=\epsilon \sum_{\alpha, i} C_{\alpha, i} u^{\alpha} e_{i} \\
u\left(t_{0}\right)=u_{0} .
\end{array}\right.
$$

where the smallness of $u$ is replaced by the smallness of $\epsilon$. We use the change of variable $u=y+\epsilon h(y)$. Notice that this change of variable is automatically a near identity change of variables as long as $h(y)$ is a bounded vector field.

Now we want to find a suitable $h(y)$ to cancel as many nonlinear terms as possible. The following example will guide us how we should define a suitable new variable.

Example 5.1. Consider the following system of equations

$$
\left\{\begin{array}{l}
\dot{u}+A u=\epsilon c u^{\alpha} e_{j} \\
u\left(t_{0}\right)=u_{0}
\end{array}\right.
$$

where $\alpha$ is some index, $c$ is a constant and $e_{j}$ is the column vector whose $j$ th entry is 1 otherwise zero. Let $u=y+\epsilon p y^{\alpha} e_{j}$ be the near identity change of variable, for some constant $p$. Then $\dot{u}=\dot{y}+\epsilon p D_{y}\left(y^{\alpha} e_{j}\right) \dot{y}$, where

$$
D_{y}\left[\begin{array}{c}
f_{1}(y) \\
f_{2}(y) \\
\vdots \\
f_{n}(y)
\end{array}\right]=\left[\begin{array}{cccc}
\frac{\partial f_{1}}{\partial y_{1}} & \frac{\partial f_{1}}{\partial y_{2}} & \ldots & \frac{\partial f_{1}}{\partial y_{n}} \\
\frac{\partial f_{2}}{\partial y_{1}} & \frac{\partial f_{2}}{\partial y_{2}} & \ldots & \frac{\partial f_{2}}{\partial y_{n}} \\
\vdots & \vdots & \ddots & \vdots \\
\frac{\partial f_{n}}{\partial y_{1}} & \frac{\partial f_{n}}{\partial y_{2}} & \cdots & \frac{\partial f_{n}}{\partial y_{n}}
\end{array}\right]
$$

is the matrix of partial derivatives and $y=\left(y_{1}, y_{2}, \ldots, y_{n}\right)$ and $f_{i}$ s are complex multivariate functions, for $i=1,2, \ldots, n$. Now we substitute them into the (5.3):

\footnotetext{
1 This rule is called "costyle".
} 


$$
\dot{y}+\epsilon p D_{y}\left(y^{\alpha} e_{j}\right) \dot{y}=-A\left(y+\epsilon p y^{\alpha} e_{j}\right)+\epsilon c\left(y+\epsilon p y^{\alpha} e_{j}\right)^{\alpha} e_{j} .
$$

Using the facts $(I+a \epsilon M(y))^{-1}=I-a \epsilon M(y)+\mathcal{O}\left(\epsilon^{2}\right)$ as well as $(y+\epsilon z)^{\alpha}=$ $y^{\alpha}+\mathcal{O}(\epsilon)$, where $M$ is an arbitrary $n \times n$ matrix, $a$ is a constant, $y$ and $z$ are vectors and $\alpha$ is some index, we find

$$
\begin{aligned}
\dot{y}=(I-\epsilon p D)\left(-A y-\epsilon p A y^{\alpha} e_{j}+\epsilon c y^{\alpha} e_{j}\right) & +\mathcal{O}\left(\epsilon^{2}\right) \\
= & -A y+\epsilon c y^{\alpha} e_{j}-\left[p y^{\alpha} e_{j},-A y\right]+\mathcal{O}\left(\epsilon^{2}\right),
\end{aligned}
$$

where $\left[\epsilon p y^{\alpha} e_{j},-A y\right]=-\epsilon p D A y+\epsilon p y^{\alpha} A e_{j}$ is the Lie derivative (see the definition 6.39) and $D=D_{y}\left(y^{\alpha} e_{j}\right)$. Now let us calculate the Lie derivative for this particular change of variable. Notice that $\left(D y^{\alpha}\right) e_{j}=\sum_{i=1}^{n} \frac{\partial y^{\alpha}}{\partial y_{i}} e_{j i}$, where $e_{l m}$ is the $n \times n$ matrix whose $(l, m)$ entry is 1 , otherwise 0 . Thus

$$
D_{y}\left(y^{\alpha} e_{j}\right) A y=\sum_{i=1}^{n} \frac{\partial y^{\alpha}}{\partial y_{i}} e_{j i} A y=\sum_{i=1}^{n} \alpha_{i} \frac{y^{\alpha}}{y_{i}} \lambda_{i} y_{i} e_{j}=y^{\alpha} \sum_{i=1}^{n} \alpha_{i} \lambda_{i} e_{j} .
$$

Hence

$$
\left[\epsilon p y^{\alpha},-A y\right]=-\epsilon p\left(\sum_{i=1}^{n} \alpha_{i} \lambda_{i}\right) y^{\alpha} e_{j}+\epsilon p y^{\alpha} A e_{j}=\epsilon p \Lambda_{\alpha, j} y^{\alpha} e_{j} .
$$

Thus (5.4) reads

$$
\dot{y}=-A y+c \epsilon y^{\alpha} e_{j}-\epsilon p \Lambda_{\alpha, j} y^{\alpha} e_{j}+\mathcal{O}\left(\epsilon^{2}\right)
$$

Notice that if $\alpha$ is a multi-index such that $\Lambda_{\alpha, j}=0$ (having a resonant term), the nonlinear term $c \epsilon y^{\alpha} e_{j}$ cannot be eliminated. However, for the multi-index $\alpha$ such that $\Lambda_{\alpha, j} \neq 0$, by choosing $p=\frac{c}{\Lambda_{\alpha, j}}$, we can cancel the nonlinear term, $c \epsilon y^{\alpha} e_{j}$.

This example gives us a good idea of what change of variable we should define to simplify nonlinear terms as much as possible. For the system (5.1), let us define

$$
u=y+\epsilon \sum_{\Lambda_{\alpha, j} \neq 0} \frac{C_{\alpha, i}}{\Lambda_{\alpha, j}} y^{\alpha} e_{i}
$$


which is the near identity change of variable. Hence by substituting (5.5) into the system (5.2) we obtain

$$
\dot{y}=-A y+\epsilon \sum_{\Lambda_{\alpha, j}=0} C_{\alpha, i} y^{\alpha} e_{i}+\mathcal{O}\left(\epsilon^{2}\right)
$$

This is the normal form of the system (5.2).

\subsection{The Relation Between NF and RG}

Recall the simplified RG approximation and RG equation (or near identity change of variable) from the previous chapter (see (4.10) and (4.11)):

$$
\begin{gathered}
u(t)=W(t)+\epsilon \sum_{\Lambda_{\alpha, i} \neq 0} \frac{C_{\alpha, i}}{\Lambda_{\alpha, i}} W(t)^{\alpha} e_{i}+\mathcal{O}\left(\epsilon^{2}\right), \\
\frac{d W}{d t_{0}}=-A W+\epsilon \sum_{\Lambda_{\alpha, i}=0} C_{\alpha, i} W^{\alpha} e_{i}+\mathcal{O}\left(\epsilon^{2}\right) .
\end{gathered}
$$

Now compare (5.5) and (5.6) to (5.7) and (5.8) respectively! The equations and near identity change of variables are exactly the same. This implies that outcomes of the RG method and NF theory are the same. The relation between RG and NF can be understood in the following commutative diagram:

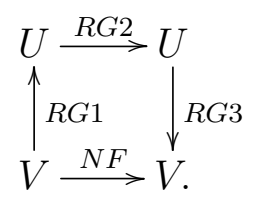

In this diagram, $V$ is the space of solutions to the system of differential equations which contain a small parameter and $U$ is the space of expansions with respect to $\epsilon$. In the NF theory, we guess a near identity change of variable. By applying the changes of variable the system converts to another system which contains the small parameter. In the diagram, this change of variable is shown by $N F$. But in the RG method, we do not need to guess the change of variable. We first postulate a naive perturbative 
expansion for the solution, and then we get a sequence of differential equations which are coefficients of powers of $\epsilon(R G 1)$. Then we renormalize the coefficients $(R G 2)$. Finally, we apply the RG condition which results in the RG system or equation which belongs to $V(R G 3)$.

In the following example we will see that by converting the equation into a system of equations and by applying the RG method to the system we will obtain a new system (the RG equation or system). Since the nonlinear part of the RG system does not necessarily vanish, solving the new system is tedious or even impossible.

Example 5.2. Consider the second order nonlinear differential equation, Rayleigh Oscillator equation, which was solved in the first chapter:

$$
\ddot{y}+y=\epsilon\left(\dot{y}-\frac{1}{3} \dot{y}^{3}\right), \quad y(0)=0, \dot{y}(0)=2 a, t>0 .
$$

Let's convert the problem to a system of first order differential equations. Define $u=y$ and $v=\dot{y}$. Hence

$$
\left\{\begin{array}{l}
\dot{u}=v \\
\dot{v}=-u+\epsilon\left(v-\frac{1}{3} v^{3}\right)
\end{array}\right.
$$

or equivalently:

$$
\left[\begin{array}{c}
\dot{u} \\
\dot{v}
\end{array}\right]=\left[\begin{array}{cc}
0 & 1 \\
-1 & 0
\end{array}\right]\left[\begin{array}{l}
u \\
v
\end{array}\right]+\epsilon\left[\begin{array}{l}
0 \\
v
\end{array}\right]-\frac{1}{3} \epsilon\left[\begin{array}{c}
0 \\
v^{3}
\end{array}\right] .
$$

To apply RG to this system, we need the matrix of coefficients to be diagonal. Because it has two distinct eigenvalues, $-i$ and $i$, it is diagonalizable. To find the diagonal form we convert the field from Real line to the Complex plain by using the change of variable

$$
z=u+i v, \text { and } \quad \bar{z}=u-i v
$$

or

$$
u=\frac{1}{2}(z+\bar{z}), \text { and } \quad v=\frac{i}{2}(\bar{z}-z) .
$$


For the ease of notation, let $\bar{z}=w$. By doing some calculations we obtain

$$
\left[\begin{array}{c}
\dot{z} \\
\dot{w}
\end{array}\right]=\left[\begin{array}{cc}
-i & 0 \\
0 & i
\end{array}\right]\left[\begin{array}{l}
z \\
w
\end{array}\right]+\frac{\epsilon}{2}\left[\begin{array}{l}
z-w-\frac{1}{12}(w-z)^{3} \\
\frac{1}{12}(w-z)^{3}+w-z
\end{array}\right]
$$

or

$$
\left[\begin{array}{c}
\dot{z} \\
\dot{w}
\end{array}\right]=\left[\begin{array}{cc}
-i & 0 \\
0 & i
\end{array}\right]\left[\begin{array}{l}
z \\
w
\end{array}\right]+\frac{i \epsilon}{2}\left[\begin{array}{l}
z-w-\frac{1}{12} w^{3}+\frac{1}{12} z^{3}-\frac{1}{4} z^{2} w+\frac{1}{4} z w^{2} \\
\frac{1}{12} w^{3}-\frac{1}{12} z^{3}+\frac{1}{4} z^{2} w-\frac{1}{4} z w^{2}-z+w
\end{array}\right]
$$

Let $c_{(i, j), k}$ be the coefficient of the monomial $z^{i} w^{j} e_{k}$. Hence

$$
\left[\begin{array}{c}
\dot{z} \\
\dot{w}
\end{array}\right]=\left[\begin{array}{cc}
-i & 0 \\
0 & i
\end{array}\right]\left[\begin{array}{l}
z \\
w
\end{array}\right]+\epsilon \sum_{i=1}^{6} \sum_{k=1}^{2} c_{\alpha_{i}, k}\left[\begin{array}{l}
z \\
w
\end{array}\right]^{\alpha_{i}} e_{k}
$$

where $\alpha_{1}=(1,0), \alpha_{2}=(0,1), \alpha_{3}=(2,1), \alpha_{4}=(1,2), \alpha_{5}=(3,0), \alpha_{6}=(0,3)$,

$$
\left\{\begin{array}{l}
-c_{\alpha_{1}, 1}=c_{\alpha_{2}, 1}=c_{\alpha_{1}, 2}=-c_{\alpha_{2}, 2}=2 i \\
-c_{\alpha_{3}, 1}=c_{\alpha_{4}, 1}=c_{\alpha_{3}, 2}=-c_{\alpha_{4}, 2}=-\frac{i}{2} \\
-c_{\alpha_{5}, 1}=c_{\alpha_{6}, 1}=c_{\alpha_{5}, 2}=-c_{\alpha_{6}, 2}=\frac{i}{6}
\end{array}\right.
$$

otherwise $c_{(i, j), k}=0$.

To find the resonant terms, let $\Lambda=\left(\lambda_{1}, \lambda_{2}\right)=(-i, i)$. Hence

$$
\left\{\begin{array}{l}
<\Lambda, \alpha_{1}>=<(-i, i),(1,0)>=\lambda_{1} \\
<\Lambda, \alpha_{2}>=<(-i, i),(0,1)>=\lambda_{2} \\
<\Lambda, \alpha_{3}>=<(-i, i),(2,1)>=\lambda_{1} \\
<\Lambda, \alpha_{4}>=<(-i, i),(1,2)>=\lambda_{2} \\
<\Lambda, \alpha_{5}>=<(-i, i),(3,0)>=-3 i \\
<\Lambda, \alpha_{6}>=<(-i, i),(0,3)>=3 i
\end{array}\right.
$$

The terms that contain $V^{\alpha_{1}}, V^{\alpha_{2}}, V^{\alpha_{3}}$ and $V^{\alpha_{4}}$ are resonant terms and the terms that contain $V^{\alpha_{5}}$ and $V^{\alpha_{6}}$ are nonresonant terms. Let us define $\Lambda_{\alpha_{5}, 1}=-3 i-(-i)=$ 
$-2 i, \Lambda_{\alpha_{6}, 1}=3 i-(-i)=, \Lambda_{\alpha_{5}, 2}=-3 i-(i)=-4 i, \Lambda_{\alpha_{6}, 2}=3 i-(i)=2 i$. Hence the solution to $(5.9)$ is

$$
\left[\begin{array}{c}
z \\
w
\end{array}\right]=W+\epsilon \sum_{i=5}^{6} \sum_{k=1}^{2} \frac{c_{\alpha_{i}, k}}{\Lambda_{\alpha_{i}, k}} e^{t \Lambda_{\alpha_{i}, k}} W^{\alpha_{i}} e_{k},
$$

where $W$ satisfies the equation

$$
\dot{W}=\left[\begin{array}{cc}
-i & 0 \\
0 & i
\end{array}\right] W+\epsilon \sum_{i=1}^{4} \sum_{k=1}^{2} c_{\alpha_{i}, k} W^{\alpha_{i}} e_{k}
$$

As you are observing, by changing the scalar differential equation into a system of differential equations, we still need to solve the equation (5.10) which is not trivial. In an especial case, when the nonlinear component does not have any resonance term, the equation (5.10) is a linear system which can be solved easily. An example of such cases is given below. (Also see the corollary 5.6 (The Continuous Linearization Theorem).)

Example 5.3. Consider the linear system

$$
\left\{\begin{array}{l}
\dot{u}=u+\epsilon u \\
\dot{v}=-v+\epsilon u v
\end{array}\right.
$$

where $u_{0}=u(0)=1$ and $v_{0}=v(0)=1$. We change the system to the matrix form:

$$
\left[\begin{array}{c}
\dot{u} \\
\dot{v}
\end{array}\right]=\left[\begin{array}{cc}
1 & 0 \\
0 & -1
\end{array}\right]\left[\begin{array}{l}
u \\
v
\end{array}\right]+\epsilon\left[\begin{array}{l}
u \\
0
\end{array}\right]+\epsilon\left[\begin{array}{c}
0 \\
u v
\end{array}\right] .
$$

Let $\alpha_{1}=(1,0), \alpha_{2}=(1,1), \Lambda=(1,-1)$ and finally $c_{(i, j), k}$ be the coefficient of $u^{i} v^{j} e_{k}$, we have

$$
\left[\begin{array}{c}
\dot{u} \\
\dot{v}
\end{array}\right]=\left[\begin{array}{cc}
1 & 0 \\
0 & -1
\end{array}\right]\left[\begin{array}{l}
u \\
v
\end{array}\right]+\epsilon \sum_{i=1}^{2} \sum_{k=1}^{2} c_{\alpha_{i}, k}\left[\begin{array}{l}
u \\
v
\end{array}\right]^{\alpha_{i}} e_{k}
$$

Note that

$$
\begin{gathered}
c_{\alpha_{1}, 1}=1, \Lambda_{\alpha_{1}, 1}=\lambda_{1}-<\Lambda, \alpha_{1}>=0 \\
c_{\alpha_{2}, 2}=1, \Lambda_{\alpha_{2}, 2}=\lambda_{2}-<\Lambda, \alpha_{2}>=1 .
\end{gathered}
$$


and $c_{\alpha_{i}, k}=0$, otherwise.

Similar to the previous example, the solution to the system is

$$
\left[\begin{array}{l}
u \\
v
\end{array}\right]=W+\epsilon \frac{c_{\alpha_{2}, 2}}{\Lambda_{\alpha_{2}, 2}} W^{\alpha_{2}} e_{2}
$$

where $W=\left[\begin{array}{l}W_{0} \\ W_{1}\end{array}\right]$ is the solution to the RG equation,

$$
\left[\begin{array}{c}
\dot{W}_{0} \\
\dot{W}_{1}
\end{array}\right]=\left[\begin{array}{cc}
1 & 0 \\
0 & -1
\end{array}\right]\left[\begin{array}{c}
W_{0} \\
W_{1}
\end{array}\right]+\epsilon\left[\begin{array}{c}
W_{0} \\
0
\end{array}\right] .
$$

Fortunately we can solve the RG equation:

$$
\left[\begin{array}{c}
W_{0} \\
W_{1}
\end{array}\right]=\left[\begin{array}{c}
w_{0} e^{(1+\epsilon) t} \\
w_{1} e^{-t}
\end{array}\right]
$$

where

$$
\left[\begin{array}{l}
1 \\
1
\end{array}\right]=\left[\begin{array}{l}
w_{0} \\
w_{1}
\end{array}\right]-\epsilon\left[\begin{array}{c}
0 \\
w_{0} w_{1}
\end{array}\right]
$$

Hence

$$
\left[\begin{array}{l}
W_{0} \\
W_{1}
\end{array}\right]=\left[\begin{array}{c}
e^{(1+\epsilon) t} \\
\frac{1}{1-\epsilon} e^{-t}
\end{array}\right] .
$$

Therefore, according to (5.12), the approximation to the solution of the system (5.11) reads

$$
\left[\begin{array}{l}
u \\
v
\end{array}\right]=\left[\begin{array}{c}
e^{(1+\epsilon) t} \\
\frac{e^{-t}-\epsilon e^{\epsilon t}}{1-\epsilon}
\end{array}\right]
$$

Remark 5.4. Consider a nonautonomous system of differential equations

$$
\dot{u}+A u=\epsilon g(t, u) .
$$

The RG method as well as the NF theory can be applied to this system. The only difference between applying RG to time-dependent or to time-independent systems 
is that we need an equivalent notion of the secular and resonant terms. In this case, as well, the only terms which appear in the RG equation are resonant terms. This shows that NF theory and RG method have the same relationship in the case of the nonautonomous system of equations (see [16].) An example of a nonautonomous system of differential equations is the Mathieu equation:

$$
\left[\begin{array}{l}
\dot{u} \\
\dot{v}
\end{array}\right]=\left[\begin{array}{ll}
0 & 1 \\
a & 0
\end{array}\right]\left[\begin{array}{l}
u \\
v
\end{array}\right]+2 \epsilon \cos (t)\left[\begin{array}{l}
0 \\
1
\end{array}\right] .
$$

\subsection{The Geometric Interpretations of RG and NF}

We have seen two different interpretations for the RG condition so far:

- The final solution (or the multiplicative renormalization constant) is independent of the artificially added parameter which implies $\frac{\partial y}{\partial \sigma}=\mathcal{O}\left(\epsilon^{2}\right)$.

- The naive perturbative expansion results in $V(\sigma)+\epsilon(t-\sigma) R(V(\sigma))+\ldots$. We interpret this as two first terms of the Taylor expansion of some function $V$ around the point $t=\sigma$. This yields the RG equation.

In this section, we present a new interpretation for the NF and RG procedures. There are some other geometric interpretations for RG in [13, 14].

Let the autonomous system of differential equations

$$
\dot{u}=G(u, \epsilon)
$$

be given where $G(u, \epsilon)=A u+\epsilon F(u)$, such that $A$ is a matrix and $F$ is a nonlinear vector field. Let $\psi(u)=y$ is the near identity change of variable such that $\psi$ is a diffeomorphism. (See the definition of a diffeomorphism in 6.29.) Taking derivative with respect to $t$ gives

$$
D_{u} \psi \dot{u}=\dot{y}
$$


Hence

$$
\dot{y}=\left(D_{\psi^{-1}(y)} \psi\right) G\left(\psi^{-1}(y), \epsilon\right),
$$

is a normal form of the system (5.13).

Therefore

$$
\psi_{*}(G(u, \epsilon))=\left(D_{\psi^{-1}(y)} \psi\right) G\left(\psi^{-1}(y), \epsilon\right)
$$

is the pushforward of the vector field $G(u, \epsilon)$ under the near identity change of co-

ordinates $y=\psi(u)$. (See the definition of the pushforward of a vector field under a diffeomorphism in 6.38.)

The following theorem states that under a special circumstances a system can be converted to a linear system:

Theorem 5.5. Smooth Linearization Theorem

Let $X$ and $Y$ be $C^{\infty}$ vector fields on $\mathbb{R}^{n}$, with 0 as a hyperbolic equilibrium point. Also suppose that there exists a transformation: $\left(\mathbb{R}^{n}, 0\right) \rightarrow\left(\mathbb{R}^{n}, 0\right)$ taking the Taylor series of $X$ at 0 to that of $Y$. Then there exists a local $C^{\infty}$-diffeomorphism $\psi$ : $\left(\mathbb{R}^{n}, 0\right) \rightarrow\left(\mathbb{R}^{n}, 0\right)$, such that $\psi_{*}(X)=Y$. (See [23].)

Corollary 5.6. Continuous Linearization Theorem

Let $X$ be $C^{\infty}$ vector fields on $\mathbb{R}^{n}$, with 0 as a hyperbolic equilibrium point. Then there exists a local $C^{\infty}$-diffeomorphism $\psi:\left(\mathbb{R}^{n}, 0\right) \rightarrow\left(\mathbb{R}^{n}, 0\right)$, such that $\psi_{*}(X)=F=$ Ax, where $A$ is the matrix coefficients of linear part of $X$. (See [23].)

Now, to see the geometric interpretation of the $\mathrm{RG}$ procedure, consider the system

$$
\dot{u}\left(t, t_{0}, w\left(t_{0}\right)\right)=G\left(u\left(t, t_{0}, w\left(t_{0}\right)\right), \epsilon\right),
$$

where $t_{0}$ is an arbitrary initial time and $w\left(t_{0}\right)$ is the integral constant with respect to $t_{0}$.

We rewrite the system (5.13) as

$$
\dot{u}\left(t, t_{0}, \phi\left(W\left(t_{0}\right)\right)\right)=G\left(u\left(t, t_{0}, \phi\left(W\left(t_{0}\right)\right)\right), \epsilon\right),
$$


where $\phi: \mathbb{R}^{n} \rightarrow \mathbb{R}^{n}$ is a smooth diffeomorphism and $\phi\left(W\left(t_{0}\right)\right)=w_{0}$ is the near identity change of coordinates in $(5.8) .^{2}$

Thus

$$
u\left(t, t_{0}\right)=\phi(W)+\int_{t_{0}}^{t} G\left(u\left(s, t_{0}, \phi(W)\right), \epsilon\right) d s,
$$

where $W=W\left(t_{0}\right)$.

Taking derivative with respect to $t_{0}$ from both sides of (5.14) gives

$$
\frac{d u}{d t_{0}}=\left(D_{W} \phi\right) \frac{d W}{d t_{0}}-G\left(u\left(t_{0}, t_{0}, \phi(W)\right), \epsilon\right)+\int_{t_{0}}^{t} D_{u} G\left(u\left(s, t_{0}, \phi(W)\right), \epsilon\right) \frac{d u}{d t_{0}} d s .
$$

Using the RG condition $\frac{d u}{d t_{0}}=\mathcal{O}\left(\epsilon^{2}\right)$ yields

$$
\frac{d W}{d t_{0}}=\left(D_{W} \phi\right)^{-1} G(\phi(W), \epsilon)
$$

By setting $t_{0}=t$ we obtain

$$
\phi^{*}(G(u(t, \phi(W(t))), \epsilon))=\left(D_{W(t)} \phi\right)^{-1} G(\phi(W(t), \epsilon)),
$$

the pullback of the vector field $G$ under the diffeomorphism $\phi$. (See the definition of the pullback of a vector field under a diffeomorphism in 6.38.)

Example 5.7. To see these abstract facts in practice, we consider the simplified RG procedure. Recall the system of equations

$$
\left\{\begin{array}{l}
\dot{u}+A u=\epsilon \sum_{\alpha, i} C_{\alpha, i} u^{\alpha} e_{i} \\
u\left(t_{0}\right)=u_{0}
\end{array}\right.
$$

The vector field associated with this system is $G(u, \epsilon)=-A u+\epsilon \sum_{\alpha, i} C_{\alpha, i} u^{\alpha} e_{i}$. The near-identity change of variable

$$
u_{0}=W\left(t_{0}\right)+\epsilon \sum_{\Lambda_{\alpha, i} \neq 0} \frac{C_{\alpha, i}}{\Lambda_{\alpha, i}} W\left(t_{0}\right)^{\alpha} e_{i}
$$

\footnotetext{
${ }^{2}$ Note that $\phi$ is interpreted as the relationship between $u_{0}$, the arbitrary initial value for the time $t_{0}$ and $W\left(t_{0}\right)$ which is an integration constant.
} 
is the smooth map $\phi$. The vector field of the system

$$
\begin{aligned}
\frac{d W}{d t_{0}} & =-A W+\epsilon \sum_{\Lambda_{\alpha, i}=0} C_{\alpha, i} W^{\alpha} e_{i}+\mathcal{O}\left(\epsilon^{2}\right), \\
W\left(t_{0}\right) & =\text { inverse of the change of variable (5.16), }
\end{aligned}
$$

is the pullback of the vector field (5.15) under the near-identity change of variable (5.16). 


\section{Chapter 6}

\section{APPENDIX}

\subsection{Asymptotic Analysis (Local Analysis)}

In this section we are going to have several basic definitions and notions of the local analysis of the solutions to the differential equations, see [3] chapter 3.

In Mathematics, Asymptotic Analysis describes the limiting behavior of functions near some point(s). For example, for the function $f(x)=x^{2}+3 x$, we say $f(x)$ is

asymptotic to $x^{2}$ as $x \rightarrow+\infty$ because $\lim _{x \rightarrow+\infty} \frac{x^{2}+3 x}{x^{2}}=1$. In approximating the solutions of differential equations, the asymptotic analysis is a powerful and useful tool. There is no much information in saying that the function $f$ (or the sequence $f_{n}$ ) approaches to zero. Indeed, "approximating asymptotically" is a more powerful notion than "approaching". This is why we use asymptotic analysis to distinguish the behavior of differential equations near a point at which the equations have essential singularity behaviors. For example, there is more information in finding the asymptotic behavior of solution of a differential equation near an irregular singular point relative to realizing that a solution to the differential equation approaches to infinity near an essential singular point.

Definition 6.1. Asymptotic Relations

We say $f(x)$ is asymptotic to $g(x)$ as $x \rightarrow x_{0}$ when

$$
\lim _{x \rightarrow x_{0}} \frac{f(x)}{g(x)}=1,
$$


and we write

$$
f(x) \sim g(x)
$$

Definition 6.2. The Method of Dominant Balance

Method of using asymptotic relations (near some point) in an ODE to find the leading behavior of the solutions (near the same point), is called Method of Dominant Balance. In this method, we try to simplify the equation by eliminating terms that may be negligible, having the following procedure

- We drop all terms that seem to asymptotically be very small.

- We replace the $\operatorname{sign}=$ instead of $\operatorname{sign} \sim$ and solve the ODE exactly.

- We check this case whether is consistent or not. If it is consistent, we must check that the equation from which we factor off the dominant balance solution has much less varying solution. If this happens, we say that the dominant balance solution represents the controlling factor of the exact solution.

Remark 6.3. Let $y(x)$ be the solution to a differential equation which has an irregular singular point at $x_{0}$. To approximate $y(x)$ we make the Ansatz

$$
y(x) \sim e^{S(x)}, \quad x \rightarrow x_{0}
$$

Hence we obtain a new differential equation whose solution is $S(x)$. Then by using the method of dominant balance, we may be able to drop off some of terms and eventually find an approximation for $S$ near $x_{0}$. Substituting back into the (6.2) gives a local approximation at $x_{0}$.

Remark 6.4. By viewing the leading factor of the solutions to different differential equations near irregular singular points, we come to realize that most of the times, the leading factors have exponential behaviors. Then the substitution $y \sim e^{S}$ determines the leading behavior of the solution of the differential equation. If a differential 
equation has an essential singularity at $x=x_{0}$, one may suggest $y \sim e^{\left(x-x_{0}\right)^{-b}}$, $b>0$, as the leading term of the solution. Hence $S=\left(x-x_{0}\right)^{-b}$. Now comparing $S^{\prime} \sim\left(x-x_{0}\right)^{-b-1}$ and $S^{\prime \prime} \sim\left(x-x_{0}\right)^{-b-2}$ near $x_{0}$, justifies making the assumption $S^{\prime \prime} \ll\left(S^{\prime}\right)^{2}$.

\subsection{Airy Functions}

The second order linear differential equation

$$
y^{\prime \prime}(x)=x y(x)
$$

is called the Airy equation. Let us use the Laplace transformation on a contour $L$ to solve this equation: $y(x)=\int_{L} e^{x t} \phi(t) d t$. Then

$$
\int_{L} t^{2} e^{x t} \phi(t) d t=\int_{L} x e^{x t} \phi(t) d t=\left(\phi(t) e^{x t}\right)_{\partial L}-\int_{L} e^{x t} \phi^{\prime}(t) d t
$$

We choose the contour $L$ such that $\left(\phi(t) e^{x t}\right)_{\partial L}=0$. Now we try to find the $\phi$ :

$$
\int_{L}\left(t^{2} \phi(t)+\phi^{\prime}(t)\right) e^{x t} d t=0
$$

It suffices to have $t^{2} \phi(t)+\phi^{\prime}(t)=0$. Thus $\phi=c e^{-\frac{t^{3}}{3}}$, for some constant $c$. Hence

$$
y(x)=c \int_{L} e^{x t-\frac{t^{3}}{3}} d t .
$$

For the $x$ fixed, the value of $-\frac{t^{3}}{3}$ dominates the behavior of the function inside the integral. If $L$ is a closed contour enclosing a compact region of $\mathbb{C}$, then $y(x)=0$. So $L$ has to extend to infinity in the complex plane of $t$ and in order to have a convergent integral, we must have $\operatorname{Re}\left(\frac{t^{3}}{3}\right)>0$. We write $t=|t| e^{i \theta}$. Thus

$$
\cos (3 \theta)>0 .
$$

Thus the contour $L$ has to go to infinities in one of the four regions: $\{ \pm \theta: 0<$ $\left.\theta<\frac{\pi}{6}\right\} \bigcup\left\{ \pm \theta: \frac{\pi}{2}<\theta<\frac{2 \pi}{3}\right\}$. 
We choose $L_{1}=\{$ is : $-\infty<s<\infty\}$ and $\{i u:-\infty<u<0\} \bigcup\{v>0\}$. In the first case we have:

$$
\begin{array}{r}
y(x)=c \int_{-\infty}^{\infty} e^{i\left(x s+\frac{s^{3}}{3}\right)}(i d s)=c i \int_{-\infty}^{\infty} \cos \left(x s+\frac{s^{3}}{3}\right)+i \sin \left(x s+\frac{s^{3}}{3}\right) d s= \\
2 c i \int_{0}^{\infty} \cos \left(x s+\frac{s^{3}}{3}\right) d s .
\end{array}
$$

In the second case we have:

$$
\begin{gathered}
y(x)=c\left(\int_{0}^{\infty} e^{-i\left(x u+\frac{u^{3}}{3}\right)}(-i d u)+\int_{0}^{\infty} e^{\left(x v-\frac{v^{3}}{3}\right)} d v\right)= \\
c \int_{0}^{\infty} e^{\left(x v-\frac{v^{3}}{3}\right)}-i \cos \left(x v+\frac{v^{3}}{3}\right)-\sin \left(x v+\frac{v^{3}}{3}\right) d v .
\end{gathered}
$$

Since any linear combination of two linearly independent solution of a linear differential equation is still a solution, we can simplify the second solution to the Airy equation.

If we name the first solution $\operatorname{Ai}(x)$ (Airy function of first kind) and the second independent solution $\operatorname{Bi}(x)$ (Airy function of second kind), we have

$$
\begin{aligned}
& \operatorname{Ai}(x)=C_{1} \int_{0}^{\infty} \cos \left(\frac{t^{3}}{3}+x t\right) d t \\
& \operatorname{Bi}(x)=C_{2} \int_{0}^{\infty}\left(e^{\frac{-t^{3}}{3}+x t}+\sin \left(\frac{t^{3}}{3}+x t\right)\right) d t
\end{aligned}
$$

for some arbitrary the constants $C_{2}$ and $C_{2}$. (See [27].)

The Airy Function of first kind, Ai $(x)$, remains well-defined for $x$ arbitrary. To see why, without loss of generality, let us assume $x=0$. The integral is well-defined as an improper integral if

$$
I=\int_{c}^{\infty} \cos \left(\frac{t^{3}}{3}\right) d t=\lim _{p \rightarrow \infty} \int_{c}^{p} \cos \left(\frac{t^{3}}{3}\right) d t
$$

for some positive $c$, is well-defined; because

$$
\int_{0}^{\infty} \cos \left(\frac{t^{3}}{3}\right) d t=\int_{0}^{c} \cos \left(\frac{t^{3}}{3}\right) d t+\int_{c}^{\infty} \cos \left(\frac{t^{3}}{3}\right) d t
$$


Note that the first integral in the right hand side exists and then the problem depends on whether or not the second integral in the right hand side exists. suppose that $c=\sqrt[3]{3 \pi}$. Thus

$$
\begin{aligned}
& \left|\int_{\sqrt[3]{3 \pi}}^{p} \cos \left(\frac{t^{3}}{3}\right) d t\right|=\left|\int_{\sqrt[3]{3 \pi}}^{p} \frac{1}{t^{2}} t^{2} \cos \left(\frac{t^{3}}{3}\right) d t\right|=\left|\frac{\sin \left(\frac{p^{3}}{3}\right)}{p^{2}}+2 \int_{\sqrt[3]{3 \pi}}^{p} \frac{\sin \left(\frac{t^{3}}{3}\right)}{t^{3}} d t\right| \leq \\
& \left|\frac{\sin \left(\frac{p^{3}}{3}\right)}{p^{2}}\right|+2 \int_{\sqrt[3]{3 \pi}}^{p}\left|\frac{\sin \left(\frac{t^{3}}{3}\right)}{t^{3}}\right| d t \leq\left|\frac{\sin \left(\frac{p^{3}}{3}\right)}{p^{2}}\right|+2 \int_{\sqrt[3]{3 \pi}}^{p} \frac{d t}{t^{3}} \leq\left|\frac{\sin \left(\frac{p^{3}}{3}\right)}{p^{2}}\right|-\frac{1}{p^{2}}+\frac{1}{\sqrt[3]{9 \pi^{2}}} .
\end{aligned}
$$

Note that we have used integration by parts in the second equality and have applied the triangle inequality twice.

By taking limit at infinity we have

$\left|\lim _{p \rightarrow \infty} \int_{\sqrt[3]{3 \pi}}^{p} \cos \left(\frac{t^{3}}{3}\right) d t\right|=\lim _{p \rightarrow \infty}\left|\int_{\sqrt[3]{3 \pi}}^{p} \cos \left(\frac{t^{3}}{3}\right) d t\right| \leq \lim _{p \rightarrow \infty}\left(\left|\frac{\sin \left(\frac{p^{3}}{3}\right)}{p^{2}}\right|-\frac{1}{p^{2}}+\frac{1}{\sqrt[3]{9 \pi^{2}}}\right)=\frac{1}{\sqrt[3]{9 \pi^{2}}}$.

Therefore the improper integral (6.11) is absolutely convergent which implies it is convergent.

In the rest of this subsection, we are going to approximate the solutions to the Airy equation assuming that $y(x) \sim e^{S(x)}$ (see [3].) By substituting this into the Airy equation, we will find a new equation with respect to $S(x)$ :

$$
\left(S^{\prime}\right)^{2}+S^{\prime \prime}=x, x \rightarrow \infty
$$

Since $x=\infty$ is an irregular singular point, we may assume $S^{\prime \prime} \ll\left(S^{\prime}\right)^{2}$. Hence

$$
\left(S^{\prime}\right)^{2} \sim x, x \rightarrow \infty
$$

and thus $S \sim \pm \frac{2}{3} x^{\frac{3}{2}}$.

Then $y \sim e^{\frac{2}{3} x^{\frac{3}{2}}}$ and $y \sim e^{\frac{-2}{3} x^{\frac{3}{2}}}$ are controlling factors of $\operatorname{Bi}(x)$ and $\operatorname{Ai}(x)$, respectively. 
Notice that to get a better approximation (to obtain the leading behavior) we can repeat this procedure. To do this, we make the Ansatz

$$
S \sim \pm \frac{2}{3} x^{\frac{3}{2}}+T(x) \text { s.t } T \ll x^{\frac{3}{2}} \text { as } x \rightarrow \infty
$$

Inserting this into the Airy equation gives

$$
\pm \frac{1}{2} x^{\frac{-1}{2}}+T^{\prime \prime}+\left( \pm x^{\frac{1}{2}}+T^{\prime}\right)^{2}=x
$$

Hence

$$
\pm \frac{1}{2} x^{\frac{-1}{2}}+T^{\prime \prime} \pm 2 x^{\frac{1}{2}} T^{\prime}+\left(T^{\prime}\right)^{2}=0
$$

The assumption $T \ll x^{\frac{3}{2}}$ justifies the following inequalities

$$
\begin{aligned}
T^{\prime} & \ll x^{\frac{1}{2}}, \\
\left(T^{\prime}\right)^{2} & \ll x^{\frac{1}{2}} T^{\prime}, \\
T^{\prime \prime} & \ll x^{\frac{-1}{2}} .
\end{aligned}
$$

Thus (6.16) reduces to

$$
\pm \frac{1}{2} x^{\frac{-1}{2}} \pm 2 x^{\frac{1}{2}} T^{\prime} \sim 0
$$

Hence

$$
T \sim \ln \left(x^{\frac{-1}{4}}\right)
$$

Therefore the leading terms of two independent solution to (6.3) are

$$
\begin{aligned}
& y \sim c_{1} x^{\frac{-1}{4}} e^{\frac{2}{3} x^{\frac{3}{2}}}, \quad x \rightarrow \infty, \\
& y \sim c_{2} x^{\frac{-1}{4}} e^{-\frac{2}{3} x^{\frac{3}{2}}}, \quad x \rightarrow \infty .
\end{aligned}
$$

It can be shown that $c_{1}=\frac{1}{2} \pi^{\frac{-1}{2}}$ and $c_{2}=\pi^{\frac{-1}{2}}$. Hence 


$$
\begin{aligned}
& \mathrm{Ai} \sim \frac{1}{2} \pi^{\frac{-1}{2}} x^{\frac{-1}{4}} e^{-\frac{2}{3} x^{\frac{3}{2}}}, \quad x \rightarrow \infty, \\
& \mathrm{Bi} \sim \pi^{\frac{-1}{2}} x^{\frac{-1}{4}} e^{\frac{2}{3} x^{\frac{3}{2}}}, \quad x \rightarrow \infty .
\end{aligned}
$$

Remark 6.5. Let $w$ be the Wronskian of the Airy equation. Since $w$ is a constant and since $w(\infty)=\frac{1}{\pi}$, we have

$$
w(x)=\operatorname{Ai}(x) \operatorname{Bi}^{\prime}(x)-\operatorname{Bi}(x) \operatorname{Ai}^{\prime}(x)=\frac{1}{\pi} .
$$

Remark 6.6. Note that if we look for the behavior of the Airy functions near the other irregular singular point, $x=-\infty$, as above we find $S= \pm i \frac{2}{3}(-x)^{\frac{3}{2}}$, as $x \rightarrow-\infty$. Thus we obtain

$$
y(x)=c(-x)^{-\frac{1}{4}} e^{ \pm \frac{2}{3} i(-x)^{\frac{3}{2}}}, \quad x \rightarrow-\infty .
$$

The Airy function have trigonometric forms as follows

$$
\begin{array}{ll}
\mathrm{Ai} \sim \pi^{-\frac{1}{2}}(-x)^{-\frac{1}{4}} \sin \left(\frac{2}{3}(-x)^{\frac{3}{2}}+\frac{1}{4} \pi\right), & x \rightarrow-\infty, \\
\mathrm{Bi} \sim \pi^{-\frac{1}{2}}(-x)^{-\frac{1}{4}} \cos \left(\frac{2}{3}(-x)^{\frac{3}{2}}+\frac{1}{4} \pi\right), \quad x \rightarrow-\infty .
\end{array}
$$

(See [3].)

\subsection{Perturbation Theory}

We are going to recall several basic definitions, notions and methods of the global analysis of solutions to the ordinary differential equations, see [3] part III.

Definition 6.7. Perturbation theory is a collection of methods which can be applied to equations containing a small parameter (say $\epsilon$ ) to approximate the solutions. In Perturbation theory, we make an Ansatz that the solution to the equation can be written as an expansion with respect to $\epsilon^{n}$ where $n \geq 0 .{ }^{1}$. Then we are left with

\footnotetext{
${ }^{1}$ Sometimes we need to postulate an expansion with respect to $f(\epsilon)^{n}$, where $f(\epsilon) \rightarrow 0$ as $\epsilon \rightarrow 0$ for the solution
} 
a recursive sequence of equations whose solutions are coefficients of $\epsilon^{n}$. Note that the condition on such Ansatz is that new equations should be easier to solve than the original problem. We truncate the series somewhere and try to solve remained equations. Perturbation theory is a powerful tool to approximate solutions to perturbed equations. In some cases, if a complicated equation (not perturbed) is given, one can add an $\epsilon$ somewhere, and then can apply perturbation theory to approximate the solutions to the new perturbed equation. Finally, by setting $\epsilon=1$ we obtain the solutions to the former equation.

Example 6.8. Suppose the following differential equation is given

$$
y^{\prime \prime}(x)=f(x) y, \quad y(0)=1, \dot{y}(0)=2,
$$

for some smooth function $f$. One can artificially add a small parameter into the equation and then solve it:

$$
y^{\prime \prime}(x)=\epsilon f(x) y, \quad \epsilon \ll 1
$$

We note that we can choose several places for $\epsilon$, but it is most relevant to choose a case for which the unperturbed equation is easy to solve. Now we postulate the expansion

$$
y(x)=y_{0}(x)+\epsilon y_{1}(x)+\ldots,
$$

where $y_{0}(0)=1, \dot{y}_{0}(0)=2$ and $y_{i}(0)=\dot{y}_{i}(0)=0$ for $i>0$. Now we have a recursive differential equation which are less complicated than (6.23) to be solved using the method of variation of parameters.

Definition 6.9. Oscillation Function and Oscillatory Equations

An oscillating function of $x$ (or $t$ ) is a function with infinitely many real zeros. A differential equation is said to be oscillatory if it has at least one oscillating solution. 


\subsection{Boundary Layer Theory}

Definition 6.10. Boundary Layer theory is a perturbation theory which can be applied to some classes of boundary value problems with a small parameter (say $\epsilon$ ). A differential equation whose highest derivative term is multiplied by a small parameter may have an abrupt change near some point(s) in the integration region. Such regions are called boundary layer or inner regions. In boundary layer theory we separate the region into different regions such as the inner, outer and intermediate regions. We find the solutions in the inner and outer regions and match them asymptotically in the intermediate region(s). The solutions in these regions are shown by $y_{\text {in }}, y_{\text {out }}$ and $y_{\text {match }}$ respectively. Then by the formula

$$
Y_{\text {unif }}=y_{\text {in }}+y_{\text {out }}-y_{\text {match }},
$$

we find a uniform solution which is valid throughout the region. In this method, similar to the RG method, we start with a naive perturbative expansion, with respect to $\epsilon$. But the point is that, because the highest derivative term is multiplied by an $\epsilon$, the unperturbed term loses the effect of the highest derivative term. Indeed, this depends on the boundary values: if the leading term of the naive perturbative expansion does not satisfy one of the boundary values, there must be an abrupt change near that point. The region in which the solution has an abrupt change is called the boundary layer region and the region in which the solution has a smooth behavior is called the outer region. Then the naive perturbative expansion approximates the outer region's solution. In the boundary layer theory we find the thickness of the boundary layer, say $\delta$ which is a function of $\epsilon$, and make the following Ansatz:

$$
y_{\text {in }}=y_{0}+y_{1} \delta+y_{2} \delta^{2}+\ldots
$$

However, there are examples that neither the naive perturbative expansion nor the expansion (6.25) can approximate the inner solution. We must somehow treat 
the expansion.

To find the thickness of the boundary layer, we use the change of variable $x-a=$ $\delta X$, where $a$ is the location of the boundary layer and $\delta$ depends on $\epsilon$ such that $\delta \rightarrow 0$ as $\epsilon \rightarrow 0$. Note that $X$ has a large value since $\delta$ is small. By applying the method of dominant balance, we find the thickness of the boundary layer.

Definition 6.11. (Asymptotic Matching)

The interval on which a boundary layer theory is posed, is broken into (at least) two overlapping subintervals (i.e. the inner and outer regions). We call the overlap region the Intermediate region. The approximations derived for the subinterval, should be asymptotic to each other in the intermediate region. We use perturbation theory to find an asymptotic approximation to the equation valid on this interval. However, we may fail in asymptotically matching the inner and outer solutions in the intermediate region. This turns out that the naive perturbative expansion assumed for the inner solution must be changed. Hence we have to manipulate the expansion and redo the matching.

\subsection{WKB Theory}

\section{Definition 6.12. (WKB Approximation)}

WKB theory is a method of global approximation which can be applied to linear differential equations (and may be applied to some nonlinear ones). In particular, it can be used for an equation whose highest derivative term is multiplied by a small parameter; then it contains the Boundary Layer theory as a special case. The method is to postulate the solution as an exponential function:

$$
y(x) \sim e^{\frac{1}{\delta} S(x)}, \quad \delta \rightarrow 0+,
$$

where $S(x)$ is a slowly varying function in the region in which we have dissipative or dispersive behavior. If $S$ is real, $\delta$ can be regarded as the thickness of the boundary 
layer (the dissipative case) and if it is complex, $\delta$ is the wavelength of the rapid oscillation (the dispersive case).

The Ansatz is to postulate a perturbative expansion for the exponent. Then

$$
y(x) \sim e^{\frac{1}{\delta} \sum_{n=0}^{\infty} \delta^{n} S_{n}}, \quad \delta \rightarrow 0,
$$

where $S_{n}=S_{n}(x)$.

\section{CRITERIA:}

In practice, we have to truncate the series and then to find all $S_{i}$ up to the order of the truncation. If the series is truncated in the $n+1$ st term, the following asymptotic relations must hold

$$
\begin{aligned}
\frac{1}{\delta} S_{0}(x) & \gg S_{1}(x), \quad \delta \rightarrow 0, \\
S_{1}(x) & \gg \delta S_{2}(x), \quad \delta \rightarrow 0, \\
\vdots & \\
S_{n}(x) & \gg \delta S_{n+1}(x), \quad \delta \rightarrow 0 .
\end{aligned}
$$

These conditions on $S_{i}, i \leq n$, are not sufficient to have a consistent approximation. The sufficient condition is

$$
\delta^{n} S_{n+1}(x) \ll 1, \quad \delta \rightarrow 0 .
$$

Example 6.13. Consider the Schrödinger equation

$$
\epsilon^{2} y^{\prime \prime}(x)=Q(x) y(x),
$$

where $Q(x)$ is a smooth and nowhere zero function.

By applying the WKB approximation, as we did in (2.64) we get 


$$
\begin{aligned}
& S_{0}(x)= \pm \int^{x} \sqrt{Q(t)} d t \\
& S_{1}(x)=\frac{-1}{4} \ln Q(x), \\
& S_{2}(x)= \pm \int^{x}\left(\frac{Q^{\prime \prime}}{8 Q^{\frac{3}{2}}}-\frac{5 Q^{\prime 2}}{32 Q^{\frac{5}{2}}}\right), \\
& S_{3}(x)=-\frac{Q^{\prime \prime}}{16 Q^{2}}+\frac{5 Q^{\prime 2}}{64 Q^{3}},
\end{aligned}
$$

and so on.

Remark 6.14. Consider the following perturbed linear differential equation

$$
\epsilon y^{\prime \prime}(x)+a(x) y^{\prime}(x)+b(x) y(x)=0,
$$

where $a(x)$ is differentiable and $b(x)$ is continuous. By the change of variable $Q(x)=$ $\frac{a^{2}(x)}{\epsilon^{2}}+\frac{a^{\prime}(x)}{2}+b(x)$, the problem is converted to the Schrödinger equation form

$$
\epsilon^{2} u^{\prime \prime}=Q(x) u
$$

Definition 6.15. Physical and Geometrical Approximations

In the WKB theory, approximation up to and including $\mathcal{O}\left(\frac{1}{\epsilon}\right)$ is called the geometric approximation. However, this approximation does not present a good estimate of the solutions.

Approximation up to and including $\mathcal{O}(1)$ is called the physical approximation to the equation. Different examples show that this approximation to the linear boundary layer equations is precise enough (see[3].) To have a valid Physical WKB approximation, the followings must hold:

$$
\begin{aligned}
& \frac{1}{\epsilon} S_{0} \gg S_{1}, \\
& \epsilon S_{2} \ll 1 .
\end{aligned}
$$




\subsection{Floquet Theory}

The aim of this section is to state the Floquet theorem and to present its proof. First, we present several lemmas which are necessary to prove the theorem. Thereafter, we prove the theorem in the case of a second order ordinary differential equation. Finally, we finish this section by having some remarks about the Mathieu equation.

Definition 6.16. Consider an $n \times n$ system of first order linear ordinary differential equations. The $n \times n$ matrix whose columns are the linear independent solutions to the system is called a fundamental matrix of the system.

Lemma 6.17. Consider the system of equations

$$
\dot{x}(t)=A(t) x(t)
$$

where $A(t)$ is a T-periodic $n \times n$ matrix of coefficients and $x(t) \in \mathbb{C}^{n}$. If $X(t)$ is a fundamental matrix for this system, for every invertible constant matrix $B, X(t) B$ is also a fundamental matrix.

Proof.

$$
(\dot{X} B)=(\dot{X}) B=A(X B)
$$

Since $X B$ is invertible and satisfies the system, it is a fundamental matrix.

Lemma 6.18. Same assumptions as lemma (6.17). If $X$ and $Y$ are two different fundamental matrices, then there is a constant matrix such that $Y=X B$.

Proof. Define $B(t)=X^{-1}(t) Y(t)$. Then $Y(t)=X(t) B(t)$. Hence

$$
A Y=\dot{Y}=\dot{X} B+X \dot{B}=A X B+X \dot{B}=A Y+X \dot{B}
$$

Thus $X \dot{B}=0$. Since $X$ is a fundamental solution (and then invertible), we conclude $\dot{B}=0$ which implies that $B$ is a constant matrix. 
Lemma 6.19. Same assumptions as (6.17) and let $x(t)$ be a solution to the system. Then $x(t+T)$ is also a solution to the system.

Proof. Since $x$ satisfies the system we have $\dot{x}(s)=A(s) x(s)$. This equality holds for all times $s \geq 0$, in particular for $s=t+T$, since $A$ has the period $T$. Having the periodicity of $A$ we conclude

$$
\dot{x}(t+T)=A(t) x(t+T) .
$$

We observe that $\tilde{x}(t)=x(t+T)$ satisfies the system. Note that $x$ is either a vector or matrix solution to the system.

Lemma 6.20. Same assumption as (6.17). Then there exists a vector solution $x(t)$ to the system such that $x(t+T)=\lambda x(t)$.

Proof. Let $X$ be a fundamental solution to the system. According to the lemma (6.19), $X(t+T)$ is also a fundamental matrix and by lemma 6.18, there exists a constant matrix $B$, such that $X(t+T)=X(t) B$. Let us define $x(t)=X(t) b$, where $b$ is the eigenvector corresponding to an eigenvalue of $B$, say $\lambda . x(t)$ is a solution because

$$
\dot{x}(t)=\dot{X} b=A(t) X(t) b=A(t) x(t)
$$

In addition,

$$
x(t+T)=X(t+T) b=X(t) B b=X(t) \lambda b=\lambda x(t) .
$$

Theorem 6.21. (Floquet Theorem)

Consider the linear system of differential equations

$$
\dot{x}(t)=A(t) x(t)
$$


where $A(t)$ is a T-periodic $n \times n$ matrix of coefficients and $x(t) \in \mathbb{C}^{n}$. Then there exists a solution to the system of the form $x(t)=e^{\mu t} \phi(t)$, for some complex number $\mu$ and some T-periodic function $\phi$.

Proof. Let $\left\{x_{1}, \ldots, x_{n}\right\}$ be a fundamental set of solutions (linearly independent solutions) to the system. The matrix $X(t)=\left(x_{1} x_{2} \ldots x_{n}\right)$, whose $i$ th column is $x_{i}$, is a fundamental matrix which is a solution for the system (matrix solution).

Having lemmas 6.17 to 6.19 in mind, there exists an invertible constant matrix $B$, so that $X(t+T)=X B$. Let $(\lambda, b)$ is an eigenpair of $B$. By the lemma 6.20 $x(t+T)=\lambda x(t)$. Now define $\mu$ such that $\lambda=e^{\mu T}$; also define $\phi(t)=e^{-\mu t} x(t) . \phi$ is $T$-periodic because

$$
\phi(t+T)=e^{-\mu(t+T)} x(t+T)=e^{-\mu t} e^{-\mu T} \lambda x(t)=\phi(t)
$$

We call $\lambda$ the multiplier and $\mu$ the Floquet exponent.

Remark 6.22. Floquet Theorem for the Second Order Differential Equation:

In the case of $n=2$ in the theorem 6.21, there exists another proof which is simpler: Let $y(t)$ be a general solution to a second order ODE whose coefficients are $T$-periodic. Then there exists a solution to the equation of the following form:

$$
y(t)=e^{\mu t} \phi(t)
$$

where $\mu$ is a complex number and $\phi(t)$ is a $T$-periodic function.

Proof. The equation has two linearly independent solutions; say $y_{1}(t)$ and $y_{2}(t)$. Thus any solution can be written

$$
y(t)=A y_{1}(t)+B y_{2}(t)
$$

Then

$$
y(t+T)=A y_{1}(t+T)+B y_{2}(t+T) .
$$


On the other hand, since coefficients are $T$-periodic, if $y(t)$ is a solution, so is $y(t+T)$. Therefore

$$
y_{1}(t+T)=a y_{1}(t)+b y_{2}(t), y_{2}(t+T)=c y_{1}(t)+d y_{2}(t) \text {. }
$$

Now, plugging (6.35)'s into (6.34), gives

$$
y(t+T)=A\left(a y_{1}(t)+b y_{2}(t)\right)+B\left(c y_{1}(t)+d y_{2}(t)\right)=A^{\prime} y_{1}(t)+B^{\prime} y_{2}(t),
$$

where $A^{\prime}=a A+c B$ and $B^{\prime}=b A+d B$. Then

$$
\left[\begin{array}{l}
A^{\prime} \\
B^{\prime}
\end{array}\right]=\left[\begin{array}{ll}
a & c \\
b & d
\end{array}\right]\left[\begin{array}{l}
A \\
B
\end{array}\right] .
$$

In a special case that

$$
\left[\begin{array}{l}
A \\
B
\end{array}\right]
$$

is an eigenvector of the $2 \times 2$ matrix, corresponding to eigenvalue $\lambda$, we will have

$$
y(t+T)=\lambda y(t)
$$

Let $\mu$ be the complex number such that $\lambda=e^{\mu T}$. Define $\phi(t)=e^{-\mu t} y(t)$; this function is periodic because

$$
\phi(t+T)=e^{-\mu(t+T)} y(t+T)=\left(e^{-\mu t} e^{-\mu T}\right)(\lambda y(t))=e^{-\mu t} y(t) .
$$

The last equality holds by definition of $\mu$. Hence there exist the solution

$$
y(t)=e^{\mu t} \phi(t)
$$

where $\phi$ is a $T$-periodic function. 
Remark 6.23. Floquet's theorem ensures that a solution to the Mathieu equation has the form

$$
y(t)=e^{\mu t} \phi(t),
$$

where $\mu$ is a complex value and $\phi$ is a $2 \pi$-periodic function. Since coefficients of Mathieu's equation are even functions, $\tilde{y}(t)=e^{-\mu t} \psi(t)$ is also a solution to the Mathieu equation where $\psi(t)=\phi(-t)$.

Remark 6.24. Let $y(t)$ be the general solution of an equation with $T$-periodic function and let $\mu$ be the corresponding Floquet exponent. Then $y(t)$ is unstable if Re $\mu>0$ and is stable if $R e \mu \leq 0$. In the case of a second order differential equation whose coefficients are even functions, the solutions are stable if and only if $\operatorname{Re} \mu=0$.

\subsection{Mean Value Theorem}

Theorem 6.25. Let $F: \mathbb{R}^{n} \rightarrow \mathbb{R}^{n}$ be a differentiable vector field and $\|$.$\| be the$ Euclidian norm. Let $F=\left(F_{1}, \ldots, F_{n}\right)$, where $F_{i}$ s are real valued functions on $\mathbb{R}^{n}$. Then

$$
\frac{\|F(x+y)-F(x)\|}{\|y\|} \leq \sup _{\beta \in[0,1]}\|D F(x+\beta y)\|,
$$

where $x, y$ are vector functions in $\mathbb{R}^{n}$.

Proof. Since $F_{i}$, for every $i$, is a differentiable value function on $\mathbb{R}^{n}$, we have

$$
\begin{gathered}
F_{i}(x+y)-F_{i}(x)=\int_{0}^{1} \frac{d F_{i}(x+\beta y)}{d \beta} d \beta=\int_{0}^{1} \sum_{j=1}^{n} \frac{d F_{i}(x+\beta y)}{d x_{j}} \cdot y_{j} d \beta= \\
\sum_{j=1}^{n}\left(\int_{0}^{1} \frac{d F_{i}(x+\beta y)}{d x_{j}} d \beta\right) y_{j} . \\
F(x+y)-F(x)=\left[\begin{array}{ccc}
\int_{0}^{1} \frac{d F_{1}(x+\beta y)}{d x_{1}} d \beta & \cdots & \int_{0}^{1} \frac{d F_{1}(x+\beta y)}{d x_{n}} d \beta \\
\vdots & \ddots & \vdots \\
\int_{0}^{1} \frac{d F_{n}(x+\beta y)}{d x_{1}} d \beta & \cdots & \int_{0}^{1} \frac{d F_{n}(x+\beta y)}{d x_{n}} d \beta
\end{array}\right]\left[\begin{array}{c}
y_{1} \\
\vdots \\
y_{n}
\end{array}\right]=\left(\int_{0}^{1} D F(x+\beta y) d \beta\right) y .
\end{gathered}
$$


Therefore

$$
\begin{aligned}
\|F(x+y)-F(x)\| & \leq\left\|\int_{0}^{1} D F(x+\beta y) d \beta\right\|\|y\| \leq \\
& \|y\| \int_{0}^{1} \sup _{\beta \in[0,1]}\|D F(x+\beta y)\| d \beta \leq\|y\| \sup _{\beta \in[0,1]}\|D F(x+\beta y)\| .
\end{aligned}
$$

Theorem 6.26. Let $v(s)$ be a bounded vector function on the interval $I$ and let $F(v(s))=\sum_{\alpha} c_{\alpha} v^{\alpha}(s)=\sum_{i=1}^{n}\left(\sum_{\alpha} c_{\alpha}^{i} v^{\alpha}(s)\right) e_{i}$. Then

$$
\sup _{s \in \mathbb{R}}\|D F(v(s))\| \leq M
$$

for some positive number $M$.

Proof. Since $D F$ is a matrix, it belongs to a finite dimensional vector space. Let for every $s \in \mathrm{I}, v(s)$ be bounded by some positive number. Hence

$$
\begin{aligned}
\sup _{s \in \mathbb{R}}|| D F(v(s)) \|_{1}=\sup _{s \in \mathbb{R}} \sum_{i, j}\left|\frac{\partial F_{i}(v(s))}{\partial v_{j}(s)}\right|= & \sum_{i, j}\left|\sum_{\alpha} c_{\alpha}^{i} \frac{\partial}{\partial v_{j}} v_{1}^{\alpha_{1}} \ldots v_{j}^{\alpha_{j}} \ldots v_{n}^{\alpha_{n}}\right|= \\
& \sum_{i, j}\left|\sum_{\alpha} c_{\alpha}^{i} \alpha_{j} v_{1}^{\alpha_{1}} \ldots v_{j}^{\alpha_{j}-1} \ldots v_{n}^{\alpha_{n}}\right| \leq M .
\end{aligned}
$$

Note that $v_{j}(s)=v_{j}$. Note that we used the fact that $v(s)$ and its entries, $v_{j}(s) \mathrm{s}$, are bounded!

\subsection{Gronwall's Lemma}

Lemma 6.27. Let $I=(a, b)$, with $a<b$ be an interval of the real line and let $\alpha, \beta$ and $u$ be real-valued and continuous functions on $I$.

(a) If $\beta$ is non-negative and if $u$ satisfies the integral inequality 


$$
u(t) \leq \alpha(t)+\int_{a}^{t} \beta(s) u(s) d s, \quad t \in I
$$

then

$$
u(t) \leq \alpha(t)+\int_{a}^{t} \alpha(s) \beta(s) e^{\int_{s}^{t} \beta(r) d r} d s, \quad t \in I .
$$

(b) If, in addition to the assumptions of part (a), the function $\alpha$ is a nondecreasing, then

$$
u(t) \leq \alpha(t) e^{\int_{a}^{t} \beta(s) d s}, \quad t \in I
$$

Proof. (a) Let we have

$$
u(t) \leq \alpha(t)+\int_{a}^{t} \beta(s) u(s) d s .
$$

Hence there is a nonnegative value function, $C(t)$, such that

$$
u(t)=-C(t)+\alpha(t)+\int_{a}^{t} \beta(s) u(s) d s, \quad t \in \mathrm{I} .
$$

Let us define

$$
I(t)=\int_{a}^{t} \beta(s) u(s) d s .
$$

Thus the solution to the integral equation (6.36) has the form $u(t)=-C(t)+$ $\alpha(t)+I(t)$. By substituting this into (6.36), we find

$$
I(t)=\int_{a}^{t} \beta(s)(\alpha(s)-C(s)) d s+\int_{a}^{t} \beta(s) I(s) d s .
$$

Hence $I(t)$ is the solution to the differential equation

$$
\frac{d I}{d t}=(\alpha(t)-C(t)) \beta(t)+\beta(t) I(t), \quad I(a)=0 .
$$

The solution to this differential equation is

$$
I(t)=e^{\int_{a}^{t} \beta(s) d s} \int_{a}^{t} \beta(s)(\alpha(s)-C(s)) e^{-\int_{a}^{s} \beta(r) d r} d s .
$$


Hence

$$
I(t)=\int_{a}^{t} \beta(s) \alpha(s) e^{\int_{s}^{t} \beta(r) d r} d s-\int_{a}^{t} \beta(s) C(s) e^{\int_{s}^{t} \beta(r) d r} d s .
$$

Notice that $\beta$ and $C$ are nonnegative functions which implies that the second integral is nonnegative. Substituting this into (6.36) implies

$$
u(t)=-C(t)+\alpha(t)+\int_{a}^{t} \beta(s) \alpha(s) e^{\int_{s}^{t} \beta(r) d r} d s-\int_{a}^{t} \beta(s) C(s) e^{\int_{s}^{t} \beta(r) d r} d s .
$$

Therefore

$$
u(t) \leq \alpha(t)+\int_{a}^{t} \beta(s) \alpha(s) e^{\int_{s}^{t} \beta(r) d r} d s
$$

(b) Suppose that $\alpha(s)$ is a nondecreasing function. Hence $\alpha(s) \leq \alpha(t)$, for every $s \in(a, t)$. Thus (6.37) implies that

$$
u(t) \leq \alpha(t)\left(1+\int_{a}^{t} \beta(s) e^{\int_{s}^{t} \beta(r) d r} d s\right) .
$$

Using the method of substitution, $v=-\int_{s}^{t} \beta(r) d r$, we get

$$
u(t) \leq \alpha(t) e^{\int_{a}^{t} \beta(s) d s}
$$

\subsection{Differential Geometry}

Here we are going to recall definitions of basic objects in differential geometry, see [26] and [28].

Definition 6.28. Let $X$ be a vector field on $\mathbb{R}^{n}$. The Equilibrium point $p$ is called a hyperbolic point if $X(0)=0$ and $D X(0)=A$, where $A$ is the matrix coefficients of linear part of $X$ and has no purely imaginary eigenvalue. 
Definition 6.29. Homeomorphism, Diffeomorphism

A smooth map $\psi: A \rightarrow B$, where $A, B \subset \mathbb{R}^{k}$, is said to be a homeomorphism (diffeomorphism) if it is 1-1 and onto and $\psi$ as well as its inverse are continuous (differentiable). If such map exists between two subspaces of $\mathbb{R}^{n}$, then they are said to be homeomorphic (diffeomorphic).

Theorem 6.30. Inverse Function Theorem

Let $B$ be an open subset of $\mathbb{R}^{n}$ and $f: B \rightarrow \mathbb{R}^{n}$ be a smooth map and $x_{0} \in B$. If $\operatorname{det}\left(D f\left(x_{0}\right)\right) \neq 0$, where $D f\left(x_{0}\right)$ is the matrix of derivative of $f$ at $x_{0}$, then there exists an open subset $U$ of $B$ such that $f(U)$ is an open set in $\mathbb{R}^{n}$ and $\left.f\right|_{U}: U \rightarrow f(U)$ is a diffeomorphism.

Theorem 6.31. Implicit Function Theorem

Let $B \subset \mathbb{R}^{k} \times \mathbb{R}^{n}$ be an open set and $f: B \rightarrow \mathbb{R}^{n}$ be a smooth function. Let $\left(x_{0}, y_{0}\right) \in B$ so that

$$
f\left(x_{0}, y_{0}\right)=0, \quad \operatorname{det}\left(\frac{\partial f}{\partial y}\left(x_{0}, y_{0}\right)\right) \neq 0 .
$$

Note that $\frac{\partial f}{\partial y}\left(x_{0}, y_{0}\right)$ is matrix derivative of $f\left(x_{0}, y\right)$ with respect to $y$ at the point $y=y_{0}$.

Then there exist open sets $V \subset \mathbb{R}^{k}$ and $U \subset \mathbb{R}^{n}$ and $g: V \rightarrow U$ such that $\left(x_{0}, y_{0}\right) \in U \times V \subset B$ and $g\left(x_{0}\right)=y_{0}$. In addition,

$$
f(x, y)=0 \Leftrightarrow g(x)=y, \quad \forall(x, y) \in U \times V .
$$

Definition 6.32. Let $U, V \subset \mathbb{R}^{n}$ be two open subsets. Two homeomorphisms $x$ : $U \rightarrow x(U)$ and $y: V \rightarrow y(V)$ are called $C^{\infty}$-related if the maps

$$
\begin{aligned}
& \text { yox }{ }^{-1}: x(U \cap V) \rightarrow y(U \cap V), \\
& \operatorname{xoy}^{-1}: y(U \cap V) \rightarrow x(U \cap V),
\end{aligned}
$$

are $C^{\infty}$. 
Definition 6.33. Charts and Atlases

Let $M$ be a topological subspace of $\mathbb{R}^{n}$. A family of mutually $C^{\infty}$-related homeomorphisms whose domains cover $M$ is called an atlas for $M$. Any particular member $(x, U)$ of an atlas is called a chart $(x: U \rightarrow x(U)$ is a homeomorphism.)

Definition 6.34. Smooth Manifold

Let $M \subseteq \mathbb{R}^{n}$ be a topological subspace. Let $\mathcal{A}$ be the family of all $C^{\infty}$-related charts on $M$. Then the pair $(M, \mathcal{A})$ is called a smooth manifold.

Definition 6.35. Smooth Sub-Manifold

A sub topological space $S$ of the smooth manifold $M \subseteq \mathbb{R}^{n}$ is called $m$-dimensional smooth sub-manifold if it is a subset of $\mathbb{R}^{m}$ and every point $p \in S$ has an open neighborhood diffeomorphic to an open subset of $M$.

Definition 6.36. Tangent Vector and Tangent Space at a point

Let $M \subseteq \mathbb{R}^{n}$ be a smooth $n$-dimensional sub-manifold and fix a point $p \in M$. A vector $Y \in \mathbb{R}^{n}$ is called a tangent vector of $M$ at $p$ if there is a smooth curve $\gamma: \mathbb{R} \rightarrow M$ such that

$$
\begin{aligned}
\left.\dot{\gamma}(t)\right|_{t=0} & =Y, \\
\gamma(0) & =p .
\end{aligned}
$$

The set of all tangent vectors of $M$ at $p$ is called the tangent space of $M$ at the point $p$.

Definition 6.37. Tangent Space on a Manifold

Let $M \subseteq \mathbb{R}^{n}$ be a smooth manifold. The tangent space on the manifold $M$ to be the set of vector fields on the manifold $M$. This space is denoted by $\operatorname{vec}(M)$.

Definition 6.38. Pushforward and Pullback of a Vector Field

Let $M, N \subseteq \mathbb{R}^{n}$ be two smooth manifolds and $\phi: M \rightarrow N$ be a diffeomorphism. The pushforward of $X \in \operatorname{vec}(M)$ (or $X: M \rightarrow T(M)$,) under $\phi$, carries vectors in 
the tangent space at every point in $M$ (domain of $\phi$ ) to the tangent space at every point in $N$ (codomain of $\phi)$. Namely, if $\phi_{*}(X)$ denotes the pushforward of $X$ under $\phi$, then

$$
\phi_{*}(X): N \rightarrow T(N),
$$

is a diffeomorphism and we have

$$
\phi_{*} X(y)=\left(D_{\phi^{-1}(y)} \phi\right) X\left(\phi^{-1}(y)\right), y=\phi(x) \in N, x \in M .
$$

For the diffeomorphism $\psi: N \rightarrow M$, the pullback of $X \in \operatorname{vec}(M)$ under $\psi$ carries vectors in the tangent space at every point in $M$ (codomain of $\psi$ ) to the tangent space at every point in $N$ (domain of $\psi$ ). which is denoted by $\psi^{*}$,

$$
\psi^{*}(X): N \rightarrow T(N)
$$

is a diffeomorphism and we have

$$
\psi^{*} X(x)=\left(D_{x} \psi\right)^{-1} X(\psi(x)), x \in N
$$

Definition 6.39. Let $M \subseteq \mathbb{R}^{k}, k>0$ be a smooth manifold and $X, Y \in \operatorname{vec}(M)$ be smooth vector fields on M. Then the Lie Product of $X$ and $Y$, is defined

$$
[X, Y](p)=D X(p) Y(p)-D Y(p) X(p) \in \operatorname{vec}(M) .
$$




\section{Chapter 7}




\section{BIBLIOGRAPHY}

[1] James Murdock: (1999), Perturbations; Philadelphia, Society for Industrial and Applied Mathematics.

[2] James Murdock: (2003), Normal Forms and Unfoldings for Local Dynamical Systems; New York, Springer-Verlag.

[3] Carl Bender, Steven Alan Orszag: (1999), Advanced Mathematical Methods for Scientists and Engineers; New York, Springer.

[4] Ludwig Prandtl: (1904) The Stationary Waves in a Gas Radiation; Physik in unserer Zeit, V. 5, P. 599-601.

[5] Henri Poincare: (1892) Les Method Nouvelles de la Mechanique Celeste; V 3.

[6] Michael James Lighthill: (1949) A Technique for rendering approximate solutions to physical problems uniformly valid; Philosophical magazine (A, Physics of condensed matter, defects and mechanical properties), V. 40 (7), P. 11791201.

[7] Harold Jeffreys: (1923), On certain approximate solutions of linear differential equations of the second order; Proceedings of the London Mathematical Society, V. 23, P. 428-436.

[8] Gregor Wentzel: (1926), 'Eine Verallgemeinerung der Quantenbedingungen fr die Zwecke der Wellen-mechanik; Zeits. f. Physik, V. 38, P. 518-529. 
[9] Hendrik Anthony Kramers: (1926), Wellenmechanik und halbzahlige Quantisierung; Zeits. f. Physik, V. 39, P. 828-840.

[10] Lon Nicolas Brillouin: (1926), Remarques sur la mcanique ondulatoire; Journal de physique, V. 7, P. 353-368.

[11] Lin-Yuan Chen, Nigel Goldenfeld, Yoshitsugu Oono: (1994), The renormalization group theory for global asymptotic analysis; Physical review letters, V. 73 (10), P. 1311-1315.

[12] Lin-Yuan Chen, Nigel Goldenfeld, Yoshitsugu Oono: (1996), The renormalization group and singular perturbations: Multiple-scale Analysis, Boundary Layer and Reductive Perturbation Theory. Physical review. E, V. 543 (1), P. 376-394.

[13] Teiji Kunihiro: (1995), A geometrical formulation of the renormalization group method for global analysis; Progress of Theoretical Physics, V. 94 (4), P. 503-514.

[14] Glenn Paquette: (2000), Renormalization group analysis of differential equations subject to slowly modulated perturbations; Physica A, V. 276 (12), P. 122-163.

[15] Mohammed Ziane: (2000), On a certain renormalization group method; Journal of Mathematical Physics, V. 41 (5), P. 3290-3299.

[16] Robert Edward Lee DeVille, Anthony Harkin, Matt Holzer, Kresimir Josic, TAsso Kaper: (2008), Analysis of a renormalization group method for solving perturbed ordinary differential equations, Physica D, V. 237 (8), P. 1029-1052. 
[17] Eleftherios Kirkinis: (2008), The renormalization group and the implicit function theorem for amplitude equations; Journal of Mathematical Physics, V. 49 (7), P. 1-16.

[18] Koji Nozaki, Yoshitsugu Oono: (2000), Renormalization-group theoretical reduction; Physical review. E, V. 63 (4), 046101 (18 pages).

[19] Yoshitsugu Oono, Yasuhiro Shiwa: (2012), Reductive renormalization of the phase-field crystal equation. Physical review. E, 86 (6), 061138 (12 pages).

[20] Yoshitsugu Oono: (2012), The Nonlinear World (Conceptual Analysis and Phenomenology); New York, Springer.

[21] Науото Снiва: (2009), Extension and unification of singular perturbation methods for ODEs based on the renormalization group method; SIAM Journal on Applied Dynamical Systems, V. 8 (4), P. 10661115.

[22] Majid Gazor, Pei Yu: (2010), Spectral sequences and parametric normal forms; Journal of Differential Equations, V. 252 (2), P. 1001-1031.

[23] Arnold Vladimir Igorevich: (1983), Geometrical methods in the theory of ordinary differential equations; Springer, New York, (English, Russian Original).

[24] Shlomo Sternberg: (1959) On the structure of local homeomorphisms of Euclideann-space, vol II; American journal of mathematics, V. 81 (3), P. 578605.

[25] Henk Broer: (2009), Normal forms in Perturbation theory; Encyclopedia of Complexity and Systems Science, Part 14, P. 6310-6329.

[26] Michael Spivak: (1970), A Comprehensive Introduction to Differential Geometry, Vol. 1, 3rd Edition, Houston. 
[27] Alan Jeffrey, Daniel Zwillinger: (2007), Table of Integrals; Series; and Products, Academic Press, 7th Edition.

[28] Joel Robbin, Dietmar Salamon: (1983), Lecture Note On Introduction to Differential Geometry, ETH Zürich and University of Wisconsin Madison.

[29] Carmen Chicone: (1999), Ordinary Differential Equations with Applications, Springer-Verlag, New York. 